Supporting Information for:

\title{
Synthesis of Porphyrins Bearing Hydrocarbon Tethers and Facile Covalent Attachment to $\mathrm{Si}(100)$
}

\author{
Zhiming Liu, Amir A. Yasseri, Robert S. Loewe, Andrey B. Lysenko, \\ Vladimir L. Malinovskii, Qian Zhao, Shyam Surthi, Qiliang Li, Veena Misra, \\ Jonathan S. Lindsey, and David F. Bocian
}

Table of Contents:

General experimental section $\quad$ S1

Melting point study $\quad$ S1

Noncommercial compounds $\quad$ S1

Description of the syntheses of new compounds S1-S9

$\begin{array}{ll}\text { Characterization data } & \text { S10-S77 }\end{array}$

General. ${ }^{1} \mathrm{H}(300$ or $400 \mathrm{MHz})$ and ${ }^{13} \mathrm{C}(75 \mathrm{MHz})$ NMR spectra were recorded in $\mathrm{CDCl}_{3}$ unless noted otherwise. Mass spectra of porphyrins were obtained by laser desorption mass spectrometry in the absence of a matrix (LDMS) and by high-resolution fast atom bombardment mass spectrometry (FABMS). Absorption and emission spectra were collected in toluene at room temperature. Elemental analyses were performed by Atlantic Microlab, Inc. Melting points are uncorrected. For porphyrins, a melting point onset value is given. Silica gel (Baker $40 \mu \mathrm{m}$ average particle size) was used for column chromatography. Chloroform contained $0.8 \%$ ethanol as a stabilizer. The presence of ethanol in chloroform enables $\mathrm{BF}_{3}$-ethanol cocatalysis in reactions with mesitaldehyde and pyrrole. The citations in the following sections refer to those listed in the body of the paper.

Melting Point Study. Each porphyrin was subjected to a melting-point determination. In a few cases, relatively sharp melting points $\left(\Delta \mathrm{T}=5-6^{\circ} \mathrm{C}\right)$ were observed. In most cases, the $\mathrm{mp}$ range was partially obscured (owing to concurrent sublimation and the intense optical density of the sample). For consistency, the value for the $\mathrm{mp}$ onset of each compound is reported. The $\mathrm{mp}$ onset values are as follows: $\mathbf{Z n 1}\left(275^{\circ} \mathrm{C}\right) ; \mathbf{Z n 2}\left(285^{\circ} \mathrm{C}\right) ; \mathbf{Z n 3}\left(\mathrm{dec}\right.$. at $\left.400{ }^{\circ} \mathrm{C}\right) ; \mathbf{Z n} 4(260$ $\left.{ }^{\circ} \mathrm{C}\right) ; \mathbf{Z n 5}\left(245^{\circ} \mathrm{C}\right) ; \mathbf{Z n 6}\left(270^{\circ} \mathrm{C}\right) ; \mathbf{Z n} 7\left(255^{\circ} \mathrm{C}\right) ; \mathbf{Z n 8}\left(425^{\circ} \mathrm{C}\right) ; \mathbf{Z n 9}\left(380^{\circ} \mathrm{C}\right) ; \mathbf{Z n} 10\left(370^{\circ} \mathrm{C}\right)$; Zn11 $\left(350^{\circ} \mathrm{C}\right) ; \mathbf{Z n 1 2}\left(350^{\circ} \mathrm{C}\right) ; \mathbf{Z n 1 3}\left(430^{\circ} \mathrm{C}\right) ; \mathbf{Z n 1 4}\left(435^{\circ} \mathrm{C}\right) ; \mathbf{Z n 1 5}\left(340^{\circ} \mathrm{C}\right) ; \mathbf{Z n} 16\left(250^{\circ} \mathrm{C}\right)$; Zn17 $\left(335^{\circ} \mathrm{C}\right) ; \mathbf{Z n 1 8}\left(290^{\circ} \mathrm{C}\right) ; \mathbf{Z n 1 9}\left(230^{\circ} \mathrm{C}\right) ; \mathbf{Z n 4 0}\left(>300^{\circ} \mathrm{C}\right) ; \mathbf{Z n} 41\left(310^{\circ} \mathrm{C}\right) ; \mathbf{4 5}\left(>450{ }^{\circ} \mathrm{C}\right)$.

Noncommercial Compounds. Compounds Zn1 ${ }^{6} \mathrm{Zn}_{2},{ }^{29} \mathrm{Zn} 3,{ }^{30} \mathrm{Zn} 4,{ }^{31} \mathrm{Zn} 5,{ }^{32} \mathbf{2 2},{ }^{37}$ 2,4,6-triethylbenzaldehyde, ${ }^{44} \mathbf{2 6},{ }^{43} \mathbf{2 9},{ }^{41} \mathbf{3 0},{ }^{42} \mathbf{3 2},{ }^{48} \mathbf{3 6},{ }^{41,49} \mathbf{3 8},{ }^{52}$ and $\mathbf{4 4}{ }^{55}$ were prepared as described in the literature.

Zn(II)-5,10,15-Trimesityl-5-(4-vinylphenyl)porphyrin (Zn6). Following a standard procedure for mixed-aldehyde condensation ${ }^{34}$ at high concentration ${ }^{35}$ with $\mathrm{BF}_{3} \cdot \mathrm{O}(\mathrm{Et})_{2}$-ethanol cocatalysis, ${ }^{36}$ samples of $21(500 \mathrm{mg}, 2.81 \mathrm{mmol})$, mesitaldehyde $(1.24 \mathrm{~mL}, 8.42 \mathrm{mmol})$, and pyrrole $(779 \mu \mathrm{L}, 11.2 \mathrm{mmol})$ were condensed in $\mathrm{CHCl}_{3}(153 \mathrm{~mL})$ in the presence of $\mathrm{BF}_{3} \cdot \mathrm{O}(\mathrm{Et})_{2}$ $(347 \mu \mathrm{L}, 2.74 \mathrm{mmol})$ at room temperature for $1 \mathrm{~h}$. Then DDQ $(1.91 \mathrm{~g}, 8.42 \mathrm{mmol})$ was added. 
After $10 \mathrm{~min}$, the crude mixture was passed through a silica column $\left(\mathrm{CH}_{2} \mathrm{Cl}_{2}\right)$ to recover the mixture of porphyrins free from polar byproducts. A solution of the porphyrin mixture in $\mathrm{CHCl}_{3}$ $(150 \mathrm{~mL})$ was treated with a solution of $\mathrm{Zn}(\mathrm{OAc})_{2} \cdot 2 \mathrm{H}_{2} \mathrm{O}(1.53 \mathrm{~g}, 7.00 \mathrm{mmol})$ in $\mathrm{MeOH}(20 \mathrm{~mL})$. After $15 \mathrm{~h}$, the solution was washed with water. Column chromatography [silica, $\mathrm{CHCl}_{3} /$ hexanes (11:9) followed by silica, $\mathrm{CHCl}_{3} /$ hexanes (1:2)] afforded a purple solid (330 $\mathrm{mg}, 14 \%$ ): $\mathrm{mp}$ onset $270{ }^{\circ} \mathrm{C} ;{ }^{1} \mathrm{H}$ NMR $\delta 1.85(\mathrm{~s}, 12 \mathrm{H}), 1.87(\mathrm{~s}, 6 \mathrm{H}), 2.63(\mathrm{~s}, 9 \mathrm{H}), 5.48(\mathrm{~d}, J=10.0 \mathrm{~Hz}, 1 \mathrm{H}), 6.08(\mathrm{~d}, J$ $=16.8 \mathrm{~Hz}, 1 \mathrm{H}), 7.07\left(\mathrm{dd}, J^{1}=16.8 \mathrm{~Hz}, J^{2}=10.0 \mathrm{~Hz}, 1 \mathrm{H}\right), 7.31(\mathrm{~s}, 6 \mathrm{H}), 7.82(\mathrm{~d}, J=9.6 \mathrm{~Hz}, 2 \mathrm{H})$, $8.21(\mathrm{~d}, J=9.6 \mathrm{~Hz}, 2 \mathrm{H}), 8.70-8.79(\mathrm{~m}, 6 \mathrm{H}), 8.92(\mathrm{~d}, J=5.4 \mathrm{~Hz}, 2 \mathrm{H})$; LDMS obsd 829.4; FABMS obsd 828.3176, calcd $828.3170\left(\mathrm{C}_{55} \mathrm{H}_{48} \mathrm{~N}_{4} \mathrm{Zn}\right)$; $\lambda_{\text {abs }} 423,512,550,588 \mathrm{~nm}$.

Zn(II)-5-(4-Allylphenyl)-10,15,20-trimesitylporphyrin (Zn7). Following the procedure for Zn6, reaction of $\mathbf{2 3}(365 \mathrm{mg}, 2.50 \mathrm{mmol})$, mesitaldehyde (1.11 g, $7.50 \mathrm{mmol})$, and pyrrole $(672 \mathrm{mg}, 10.0 \mathrm{mmol})$ was carried out in $\mathrm{CHCl}_{3}(1.0 \mathrm{~L})$ in the presence of $\mathrm{BF}_{3} \cdot \mathrm{O}(\mathrm{Et})_{2}$ (1.32 $\mathrm{mL}$ of a $2.5 \mathrm{M}$ solution in $\mathrm{CHCl}_{3}$ ) at room temperature for $1 \mathrm{~h}$ followed by oxidation with DDQ (1.70 g, $7.50 \mathrm{mmol})$ and passage through a silica pad $\left[\mathrm{CH}_{2} \mathrm{Cl}_{2} /\right.$ hexanes, (1:1)]. The mixture of porphyrins was dissolved in THF $(250 \mathrm{~mL})$ and treated with $\mathrm{Zn}(\mathrm{OAc})_{2} \cdot 2 \mathrm{H}_{2} \mathrm{O}(450$ $\mathrm{mg}, 2.10 \mathrm{mmol}$ ) at $50{ }^{\circ} \mathrm{C}$ for $4 \mathrm{~h}$ and then overnight at room temperature. The volume of THF was reduced to $50 \mathrm{~mL}$ and the mixture of zinc porphyrins was precipitated upon addition of methanol. Chromatography [silica, toluene/hexanes, (1:2.5)] followed by crystallization $\left(\mathrm{CH}_{2} \mathrm{Cl}_{2} / \mathrm{MeOH}\right)$ gave pink crystals $(250 \mathrm{mg}, 12 \%)$ : mp onset $255{ }^{\circ} \mathrm{C} ;{ }^{1} \mathrm{H} \mathrm{NMR} \delta 1.83(\mathrm{~s}, 18 \mathrm{H})$, $2.63(\mathrm{~s}, 9 \mathrm{H}), 3.75(\mathrm{~d}, J=6.4 \mathrm{~Hz} 2 \mathrm{H}), 5.30(\mathrm{~m}, 2 \mathrm{H}), 6.29-6.32(\mathrm{~m}, 1 \mathrm{H}), 7.26(\mathrm{~s}, 6 \mathrm{H}), 7.55(\mathrm{~d}, J=$ $7.2 \mathrm{~Hz}, 2 \mathrm{H}), 8.13(\mathrm{~d}, J=8.0 \mathrm{~Hz}, 2 \mathrm{H}), 8.69(\mathrm{~s}, 4 \mathrm{H}), 8.73(\mathrm{~d}, J=4.8 \mathrm{~Hz}, 2 \mathrm{H}), 8.87$ (d, $J=4.8 \mathrm{~Hz}$, $2 \mathrm{H})$; LDMS obsd 842.97; FABMS obsd 842.3365, calcd 842.3327 $\left(\mathrm{C}_{56} \mathrm{H}_{50} \mathrm{~N}_{4} \mathrm{Zn}\right) ; \lambda_{\text {abs }} 421,551$, $593 \mathrm{~nm}$.

5-Iodo-10,15,20-tri-p-tolylporphyrin (8). Following a standard method, ${ }^{50}$ a solution of $37(871 \mathrm{mg}, 1.50 \mathrm{mmol})$ and $\mathrm{I}_{2}(267 \mathrm{mg}, 1.05 \mathrm{mmol})$ in $\mathrm{CHCl}_{3}(210 \mathrm{~mL})$ was treated with a solution of [bis(trifluoroacetoxy)iodo]benzene $(478 \mathrm{mg}, 1.20 \mathrm{mmol})$ in $\mathrm{CHCl}_{3}(30 \mathrm{~mL})$ followed by pyridine $(1.3 \mathrm{~mL})$. The mixture was stirred at room temperature for $1 \mathrm{~h}$. The reaction mixture was diluted with $\mathrm{CH}_{2} \mathrm{Cl}_{2}$, washed with aqueous $\mathrm{Na}_{2} \mathrm{~S}_{2} \mathrm{O}_{3}$, water and dried $\left(\mathrm{Na}_{2} \mathrm{SO}_{4}\right)$. After concentrating the solution to a volume of $\sim 100 \mathrm{~mL}, 30 \mathrm{~mL}$ of hexanes was added. The resulting purple precipitate was filtered, washed $\left(\mathrm{CH}_{2} \mathrm{Cl}_{2}\right.$, hexanes) and dried to yield the free base porphyrin $(618 \mathrm{mg})$. The filtrate was concentrated and chromatographed (silica gel, warm toluene/hexanes $=7: 3)$, affording additional free base porphyrin $(253 \mathrm{mg})$. The total yield is 871 mg (82\%): ${ }^{1} \mathrm{H}$ NMR $\delta-2.70$ (s, 2H), 2.69-2.74 (brs, 9H), 7.53-7.59 (br m, 6H), 8.04-8.09 (br m, $6 \mathrm{H}), 8.78-8.83(\mathrm{~m}, 4 \mathrm{H}), 8.89(\mathrm{~d}, J=4.8 \mathrm{~Hz}, 2 \mathrm{H}), 9.67(\mathrm{~d}, J=4.4 \mathrm{~Hz}, 2 \mathrm{H})$; LDMS obsd 706.9; FABMS obsd 706.1613, calcd $706.1593\left(\mathrm{C}_{41} \mathrm{H}_{31} \mathrm{IN}_{4}\right) ; \lambda_{\text {abs }} 424,520,557,598,656 \mathrm{~nm}$.

Zn(II)-5-Iodo-10,15,20-tri-p-tolylporphyrin (Zn8). A solution of 8 (353 $\mathrm{mg}, 0.500$ $\mathrm{mmol})$ in THF $(60 \mathrm{~mL})$ was treated with $\mathrm{Zn}(\mathrm{OAc})_{2} \cdot 2 \mathrm{H}_{2} \mathrm{O}(1.10 \mathrm{~g}, 5.00 \mathrm{mmol})$ at room temperature for $8 \mathrm{~h}$. After removal of the solvent, the residue was chromatographed (silica gel, hexanes $/ \mathrm{CH}_{2} \mathrm{Cl}_{2},(1: 1)$ ] affording a powder that was recrystallized [(hexanes $\left./ \mathrm{CH}_{2} \mathrm{Cl}_{2}\right), 349 \mathrm{mg}$, 91\%]: mp onset $425{ }^{\circ} \mathrm{C} ;{ }^{1} \mathrm{H}$ NMR $\delta$ 2.67-2.70 (br s, 9H), 7.53-7.59 (br m, 6H), 8.01-8.06 (br m, $6 \mathrm{H}), 8.79(\mathrm{~m}, 4 \mathrm{H}), 8.87(\mathrm{~d}, J=5.6 \mathrm{~Hz}, 2 \mathrm{H}), 9.72(\mathrm{~d}, J=4.4 \mathrm{~Hz}, 2 \mathrm{H})$; LDMS obsd 770.5; FABMS obsd 768.0760, calcd 768.0728 $\left(\mathrm{C}_{41} \mathrm{H}_{29} \mathrm{IN}_{4} \mathrm{Zn}\right) ; \lambda_{\text {abs }} 429,519,556,596 \mathrm{~nm}$.

Zn(II)-5-[2-(Trimethylsilyl)ethynyl]-10,15,20-tri-p-tolylporphyrin (Zn9). A sample of 32 (473 mg, $1.00 \mathrm{mmol}$ ) was reduced following a general procedure ${ }^{39}$ and the resulting 32diol was condensed with 26 (243 mg, $1.00 \mathrm{mmol})$ in $\mathrm{CH}_{3} \mathrm{CN}(400 \mathrm{~mL})$ under TFA $(930 \mu \mathrm{L}, 12.1$ $\mathrm{mmol}$ ) catalysis at room temperature for $4 \mathrm{~min}$. Then DDQ (681 $\mathrm{mg}, 3.00 \mathrm{mmol})$ was added. 
After $1 \mathrm{~h}$, TEA $(2 \mathrm{~mL})$ was added. The mixture was concentrated and the residue was chromatographed [silica gel, hexanes $/ \mathrm{CH}_{2} \mathrm{Cl}_{2},(3: 7)$ ] affording the free base porphyrin (116 $\mathrm{mg}$, 17\%): mp onset $380{ }^{\circ} \mathrm{C} ;{ }^{1} \mathrm{H}$ NMR $\delta-2.41(\mathrm{~s}, 2 \mathrm{H}), 0.62(\mathrm{~s}, 9 \mathrm{H}), 2.71(\mathrm{~s}, 3 \mathrm{H}), 2.72(\mathrm{~s}, 6 \mathrm{H}), 7.54$ $(\mathrm{d}, J=8.0 \mathrm{~Hz}, 2 \mathrm{H}), 7.57(\mathrm{~d}, J=8.0 \mathrm{~Hz}, 4 \mathrm{H}), 8.05(\mathrm{~d}, J=8.0 \mathrm{~Hz}, 2 \mathrm{H}), 8.08(\mathrm{~d}, J=8.0 \mathrm{~Hz}, 4 \mathrm{H})$, $8.79(\mathrm{~s}, 4 \mathrm{H}), 8.92(\mathrm{~d}, J=4.4 \mathrm{~Hz}, 2 \mathrm{H}), 9.65(\mathrm{~d}, J=4.8 \mathrm{~Hz}, 2 \mathrm{H})$; LDMS obsd 676.2; FABMS obsd 676.3047, calcd 676.3022 $\left(\mathrm{C}_{46} \mathrm{H}_{40} \mathrm{~N}_{4} \mathrm{Si}\right) ; \lambda_{\text {abs }} 431,497,529,567,606,664 \mathrm{~nm}$. A sample of the free base porphyrin $(169 \mathrm{mg}, 0.250 \mathrm{mmol})$ in THF $(25 \mathrm{~mL})$ was treated with a solution of $\mathrm{Zn}(\mathrm{OAc})_{2} \cdot 2 \mathrm{H}_{2} \mathrm{O}(275 \mathrm{mg}, 1.25 \mathrm{mmol})$ in $\mathrm{MeOH}(2 \mathrm{~mL})$ at room temperature for $12 \mathrm{~h}$. Column chromatography [hexanes/ $\left.\mathrm{CH}_{2} \mathrm{Cl}_{2},(3: 7)\right]$ afforded a purple solid (136 mg, 74\%; 13\% overall yield): ${ }^{1} \mathrm{H}$ NMR $\delta 0.62$ (s, 9H), 2.70-2.73 (br s, 9H), 7.53-7.59 (m, 6H), 8.04-8.10 (m, 6H), 8.79 (s, 4H), $8.92(\mathrm{~m}, 2 \mathrm{H}), 9.65$ (m, 2H); LDMS obsd 739.8; FABMS obsd 738.2167, calcd 738.2157 $\left(\mathrm{C}_{46} \mathrm{H}_{38} \mathrm{~N}_{4} \mathrm{SiZn}\right) ; \lambda_{\text {abs }} 434,523,563,605 \mathrm{~nm}$.

Zn(II)-5,10,15-Tri-p-tolyl-20-vinylporphyrin (Zn10). A solution of Zn8 (50 mg, 60 $\mu \mathrm{mol})$ in THF (24 mL) was treated overnight with $\mathrm{Pd}\left(\mathrm{PPh}_{3}\right)_{4}(7 \mathrm{mg})$ and tributyl(vinyl)tin (190 $\mu \mathrm{L}, 600 \mu \mathrm{mol})$ at $60{ }^{\circ} \mathrm{C}$ under argon. The reaction mixture was concentrated. Column chromatography [silica, $\mathrm{CH}_{2} \mathrm{Cl}_{2}$ /hexanes $(1: 1)$ ] followed by trituration of the product with hexanes afforded a purple solid (34 mg, 77\%): mp onset $370{ }^{\circ} \mathrm{C} ;{ }^{1} \mathrm{H}$ NMR $\delta 2.72(\mathrm{~s}, 3 \mathrm{H}), 2.74(\mathrm{~s}$, $6 \mathrm{H}), 6.05(\mathrm{~d}, J=17.6 \mathrm{~Hz}, 1 \mathrm{H}), 6.49(\mathrm{~d}, J=11.6 \mathrm{~Hz}, 1 \mathrm{H}), 7.55-7.58(\mathrm{~m}, 6 \mathrm{H}), 8.08-8.10(\mathrm{~m}, 6 \mathrm{H})$, $8.95(\mathrm{~s}, 4 \mathrm{H}), 8.99(\mathrm{~d}, J=4.4 \mathrm{~Hz}, 2 \mathrm{H}), 9.16-9.23(\mathrm{~m}, 1 \mathrm{H}), 9.51(\mathrm{~d}, J=4.4 \mathrm{~Hz}, 2 \mathrm{H})$; LDMS obsd 668.8; FABMS obsd 668.1928, calcd 668.1918 $\left(\mathrm{C}_{43} \mathrm{H}_{32} \mathrm{~N}_{4} \mathrm{Zn}\right) ; \lambda_{\text {abs }} 426,554,595 \mathrm{~nm}$.

Zn(II)-5-Allyl-10,15,20-tri-p-tolylporphyrin (Zn11). A sample of 32-SnBu2 $(500 \mathrm{mg}$, $0.711 \mathrm{mmol})$ was reduced following a general procedure ${ }^{39}$ and the resulting 32-diol was condensed with 24 (132 mg, $0.711 \mathrm{mmol})$ in $\mathrm{CH}_{2} \mathrm{Cl}_{2}(264 \mathrm{~mL})$ containing $\mathrm{Yb}(\mathrm{OTf})_{3}(564 \mathrm{mg}$, $0.910 \mathrm{mmol}$ ) at room temperature for $7 \mathrm{~min}$. Then DDQ (3 equiv per dipyrromethane; $484 \mathrm{mg}$, $2.13 \mathrm{mmol})$ was added. The reaction mixture was passed over a silica column $\left(\mathrm{CH}_{2} \mathrm{Cl}_{2}\right)$. The resulting free base porphyrin was dissolved in $\mathrm{CHCl}_{3}(50 \mathrm{~mL})$ and treated with a solution of $\mathrm{Zn}(\mathrm{OAc})_{2} \cdot 2 \mathrm{H}_{2} \mathrm{O}(780 \mathrm{mg}, 3.6 \mathrm{mmol})$ in $\mathrm{MeOH}(7 \mathrm{~mL})$ at room temperature for $2 \mathrm{~h}$. Column chromatography (silica, $\mathrm{CHCl}_{3}$ ) afforded a purple solid $(207 \mathrm{mg}, 42 \%): \mathrm{mp}$ onset $350{ }^{\circ} \mathrm{C} ;{ }^{1} \mathrm{H}$ NMR $\delta 2.71(\mathrm{~s}, 3 \mathrm{H}), 2.73(\mathrm{~s}, 6 \mathrm{H}), 5.18-5.22(\mathrm{~m}, 2 \mathrm{H}), 5.77(\mathrm{~d}, 2 \mathrm{H}), 6.88(\mathrm{~m}, 1 \mathrm{H}), 7.53-7.57(\mathrm{~m}$, 6H), 8.07-8.10 (m, 6H), $8.92(\mathrm{~s}, 4 \mathrm{H}), 9.00(\mathrm{~d}, J=4.4 \mathrm{~Hz}, 2 \mathrm{H}), 9.53(\mathrm{~d}, J=4.4 \mathrm{~Hz}, 2 \mathrm{H})$; LDMS obsd 681.3; FABMS obsd 682.2084, calcd $682.2075\left(\mathrm{C}_{44} \mathrm{H}_{34} \mathrm{~N}_{4} \mathrm{Zn}\right) ; \lambda_{\text {abs }} 424,552,592 \mathrm{~nm}$.

Zn(II)-5-(3-Butenyl)-10,15,20-tri-p-tolylporphyrin (Zn12). Following the procedure for $\mathbf{Z n 1 1}$, the condensation of 32-diol (derived from 32-SnBu $2 ; 500 \mathrm{mg}, 0.711 \mathrm{mmol}$ ) and $\mathbf{2 5}$ (142 $\mathrm{mg}, 0.711 \mathrm{mmol}$ ) for $7 \mathrm{~min}$, oxidation with DDQ, passage through a silica pad $\left(\mathrm{CH}_{2} \mathrm{Cl}_{2}\right)$, and metalation with $\mathrm{Zn}(\mathrm{OAc})_{2} \cdot 2 \mathrm{H}_{2} \mathrm{O}$ followed by chromatography (silica, $\mathrm{CHCl}_{3}$ ) afforded a purple solid (213 mg, 44\%): mp onset $300{ }^{\circ} \mathrm{C} ;{ }^{1} \mathrm{H}$ NMR $\delta 2.71(\mathrm{~s}, 3 \mathrm{H}), 2.73(\mathrm{~s}, 6 \mathrm{H}), 3.32(\mathrm{~m}$, 2H), 5.07-5.17 (m, 3H), $5.77(\mathrm{~d}, 1 \mathrm{H}), 6.29(\mathrm{~m}, 1 \mathrm{H}), 7.54-7.58(\mathrm{~m}, 6 \mathrm{H}), 8.07-8.10(\mathrm{~m}, 6 \mathrm{H}), 8.92$ (s, 4H), $9.01(\mathrm{~d}, J=4.4 \mathrm{~Hz}, 2 \mathrm{H}), 9.52(\mathrm{~d}, J=4.4 \mathrm{~Hz}, 2 \mathrm{H})$; LDMS obsd 697.1, 656.0 [(M allyl $)^{+}$; FABMS obsd 696.2203, calcd $696.2231\left(\mathrm{C}_{45} \mathrm{H}_{36} \mathrm{~N}_{4} \mathrm{Zn}\right) ; \lambda_{\text {abs }} 424,513,552,591 \mathrm{~nm}$.

Zn(II)-5,10,15-Tri-p-tolyl-20-(4-vinylphenyl)porphyrin (Zn13). Following the procedure for $\mathbf{Z n 9}$, the reaction of 32-diol (derived from 32; $600 \mathrm{mg}, 1.27 \mathrm{mmol}$ ) and 27 (315 $\mathrm{mg}, 1.27 \mathrm{mmol})$ in MeCN (508 mL) containing TFA (1.17 mL, $15.2 \mathrm{mmol})$ for $3 \mathrm{~min}$ followed by oxidation with DDQ $(865 \mathrm{mg}, 3.81 \mathrm{mmol})$, neutralization with TEA $(1 \mathrm{~mL})$, and passage through a silica pad $\left(\mathrm{CH}_{2} \mathrm{Cl}_{2}\right)$ afforded the partially purified free base porphyrin. Metalation in $\mathrm{CHCl}_{3}(150 \mathrm{~mL})$ with $\mathrm{Zn}(\mathrm{OAc})_{2} \cdot 2 \mathrm{H}_{2} \mathrm{O}(640 \mathrm{mg}, 2.92 \mathrm{mmol})$ in $\mathrm{MeOH}(15 \mathrm{~mL})$ for $1 \mathrm{~h}$ and the 
standard workup including washing with $\mathrm{MeOH}$ furnished a purple solid (242 $\mathrm{mg}, 26 \%$ ): $\mathrm{mp}$ onset $430{ }^{\circ} \mathrm{C} ;{ }^{1} \mathrm{H}$ NMR $\delta 2.67(\mathrm{~s}, 9 \mathrm{H}), 5.48(\mathrm{~d}, J=10.0 \mathrm{~Hz}, 1 \mathrm{H}), 6.11(\mathrm{~d}, J=16.8 \mathrm{~Hz}, 1 \mathrm{H}), 7.10$ $\left(\mathrm{dd}, J^{1}=16.8 \mathrm{~Hz}, J^{2}=10.0 \mathrm{~Hz}, 1 \mathrm{H}\right), 7.60(\mathrm{~d}, J=8.4 \mathrm{~Hz}, 6 \mathrm{H}), 7.82(\mathrm{~d}, J=8.4 \mathrm{~Hz}, 2 \mathrm{H}), 8.11(\mathrm{~d}, J$ $=8.4 \mathrm{~Hz}, 6 \mathrm{H}), 8.21(\mathrm{~d}, J=8.4 \mathrm{~Hz}, 2 \mathrm{H}), 8.99-9.01(\mathrm{~m}, 8 \mathrm{H})$; LDMS obsd 745.2; FABMS obsd 744.2233, calcd 744.2231 $\left(\mathrm{C}_{49} \mathrm{H}_{36} \mathrm{~N}_{4} \mathrm{Zn}\right) ; \lambda_{\text {abs }} 425,551,592 \mathrm{~nm}$.

Zn(II)-5-(4-Allylphenyl)-10,15,20-tri-p-tolylporphyrin (Zn14). Following the procedure for $\mathbf{Z n 1 1}$, the condensation of 32-diol (derived from 32-SnBu $; 2.00 \mathrm{~g}, 2.84 \mathrm{mmol}$ ) and 28 (745 mg, $2.84 \mathrm{mmol})$ for $15 \mathrm{~min}$, oxidation with DDQ (1.93 g, $8.52 \mathrm{mmol})$ and passage through a silica pad $\left(\mathrm{CH}_{2} \mathrm{Cl}_{2}\right)$ afforded the free base porphyrin, which was suspended in ethanol/hexanes (1:1), sonicated for $5 \mathrm{~min}$ and then centrifuged. The ethanol/hexanes mixture was decanted and the solid was dried affording the free base porphyrin (14) (455 $\mathrm{mg}, 23 \%): \mathrm{mp}$ onset $435{ }^{\circ} \mathrm{C} ;{ }^{1} \mathrm{H}$ NMR $\delta-2.77$ (brs, 2H), $2.71(\mathrm{~s}, 9 \mathrm{H}), 3.75(\mathrm{~d}, J=7.8 \mathrm{~Hz}, 2 \mathrm{H}), 5.27-5.37$ (m, 2H), 6.26-6.33 (m, 1H), 7.55-7.58 (m, 8H), 8.10-8.16 (m, 8H), 8.86 (s, 8H); LDMS obsd 697.4; FABMS obsd 696.3265 , calcd $696.3253\left(\mathrm{C}_{50} \mathrm{H}_{40} \mathrm{~N}_{4}\right)$. Metalation of the free base porphyrin (100 $\mathrm{mg}, 0.143 \mathrm{mmol})$ in $\mathrm{CHCl}_{3}(15 \mathrm{~mL})$ with $\mathrm{Zn}(\mathrm{OAc})_{2} \cdot 2 \mathrm{H}_{2} \mathrm{O}(157 \mathrm{mg}, 0.717 \mathrm{mmol})$ in $\mathrm{MeOH}(2$ $\mathrm{mL}$ ) for $18 \mathrm{~h}$ followed by standard workup and chromatography (silica, $\mathrm{CHCl}_{3}$ ) afforded a purple solid (106 mg, 97\%): ${ }^{1} \mathrm{H}$ NMR $\delta 2.72(\mathrm{~s}, 9 \mathrm{H}), 3.76(\mathrm{~d}, J=7.8 \mathrm{~Hz}, 2 \mathrm{H}), 5.27-5.38(\mathrm{~m}, 2 \mathrm{H}), 6.25-$ $6.35(\mathrm{~m}, 1 \mathrm{H}), 7.55-7.58(\mathrm{~m}, 8 \mathrm{H}), 8.10(\mathrm{~d}, J=7.6 \mathrm{~Hz}, 6 \mathrm{H}), 8.14(\mathrm{~d}, J=8.0 \mathrm{~Hz}, 2 \mathrm{H}), 8.97(\mathrm{~s}, 8 \mathrm{H})$; LDMS obsd 759.4; FABMS obsd 758.2429, calcd $758.2388\left(\mathrm{C}_{50} \mathrm{H}_{38} \mathrm{~N}_{4} \mathrm{Zn}\right) ; \lambda_{\text {abs }} 424,511,550$, $591 \mathrm{~nm}$.

Zn(II)-5-(4-Allylphenyl)-15-mesityl-10,20-di-p-tolylporphyrin (Zn15). Following the procedure for $\mathbf{Z n 1 1}$, the condensation of 33-diol (derived from 33-SnBu $; 699 \mathrm{mg}, 0.953 \mathrm{mmol}$ ) and 28 (250 mg, $0.953 \mathrm{mmol}$ ) for $15 \mathrm{~min}$, oxidation with DDQ (649 $\mathrm{mg}, 2.86 \mathrm{mmol}$ ), passage through a silica pad $\left(\mathrm{CH}_{2} \mathrm{Cl}_{2}\right)$, and metalation with $\mathrm{Zn}(\mathrm{OAc})_{2} \cdot 2 \mathrm{H}_{2} \mathrm{O}$ followed by chromatography ( silica, $\mathrm{CHCl}_{3}$ ) afforded a purple solid $(161 \mathrm{mg}, 21 \%)$ : mp onset $340{ }^{\circ} \mathrm{C} ;{ }^{1} \mathrm{H} \mathrm{NMR} \delta 1.85(\mathrm{~s}, 6 \mathrm{H})$, $2.64(\mathrm{~s}, 3 \mathrm{H}), 2.71(\mathrm{~s}, 6 \mathrm{H}), 3.76(\mathrm{~d}, J=6.8 \mathrm{~Hz}, 2 \mathrm{H}), 5.27-5.37(\mathrm{~m}, 2 \mathrm{H}), 6.29(\mathrm{~m}, 1 \mathrm{H}), 7.29(\mathrm{~s}$, 2H), 7.55-7.58 (m, 6H), 8.11-8.16 (m, 6H), $8.79(\mathrm{~d}, J=4.8 \mathrm{~Hz}, 2 \mathrm{H}), 8.92(\mathrm{~d}, J=4.8 \mathrm{~Hz}, 2 \mathrm{H})$, 8.96 (s, 4H); LDMS obsd 828.5, 845.5; FABMS obsd 828.3151, calcd $828.3170\left(\mathrm{C}_{55} \mathrm{H}_{48} \mathrm{~N}_{4} \mathrm{Zn}\right)$. $\lambda_{\text {abs }} 425,485,513,551,592 \mathrm{~nm}$.

Zn(II)-5-(4-Allylphenyl)-10,20-di-p-tolyl-15-(2,4,6-triethylphenyl)porphyrin (Zn16). Following the procedure for $\mathbf{Z n 1 1}$, the condensation of 34-diol (derived from 34-SnBu $\mathbf{3} ; 483$ $\mathrm{mg}, 0.625 \mathrm{mmol})$ and $28(163 \mathrm{mg}, 0.625 \mathrm{mmol})$ for $30 \mathrm{~min}$, oxidation with DDQ, passage through a silica pad $\left(\mathrm{CH}_{2} \mathrm{Cl}_{2}\right)$, metalation with $\mathrm{Zn}(\mathrm{OAc})_{2} \cdot 2 \mathrm{H}_{2} \mathrm{O}$, and chromatography (silica, $\mathrm{CHCl}_{3}$ ) afforded a purple solid $(51 \mathrm{mg}, 10 \%)$ : mp onset $250{ }^{\circ} \mathrm{C} ;{ }^{1} \mathrm{H} \mathrm{NMR} \delta 0.74(\mathrm{t}, J=7.2 \mathrm{~Hz}$, $6 \mathrm{H}), 1.54(\mathrm{t}, J=7.4 \mathrm{~Hz}, 3 \mathrm{H}), 2.09$ (q, 4H), $2.72(\mathrm{~s}, 6 \mathrm{H}), 3.00(\mathrm{q}, 2 \mathrm{H}), 3.76(\mathrm{~d}, J=6.6 \mathrm{~Hz}, 2 \mathrm{H})$, 5.27-5.38 (m, 2H), 6.26-6.35 (m, 1H), $7.35(\mathrm{~s}, 2 \mathrm{H}), 7.54-7.59(\mathrm{~m}, 6 \mathrm{H}), 8.12-8.17(\mathrm{~m}, 6 \mathrm{H}), 8.80$ $(\mathrm{d}, J=4.5 \mathrm{~Hz}, 2 \mathrm{H}), 8.92(\mathrm{~d}, J=4.5 \mathrm{~Hz}, 2 \mathrm{H}), 8.97$ (s, 4H); LDMS obsd 828.5; FABMS obsd 828.3151, calcd $828.3170\left(\mathrm{C}_{55} \mathrm{H}_{48} \mathrm{~N}_{4} \mathrm{Zn}\right)$; $\lambda_{\text {abs }} 425,513,551,592 \mathrm{~nm}$.

Zn(II)-5-(4-Allylphenyl)-10,20-dimethyl-15-p-tolylporphyrin (Zn17). Following the procedure for $\mathbf{Z n 1 1}$, the condensation of 35-diol (derived from 35-SnBu $; 427 \mathrm{mg}$, $0.740 \mathrm{mmol}$ ) and 29 (175 $\mathrm{mg}, 0.741 \mathrm{mmol})$ for $30 \mathrm{~min}$, oxidation with DDQ, passage through a silica pad $\left(\mathrm{CH}_{2} \mathrm{Cl}_{2}\right)$, and metalation with $\mathrm{Zn}(\mathrm{OAc})_{2} \cdot 2 \mathrm{H}_{2} \mathrm{O}$ followed by chromatography (silica, $\left.\mathrm{CHCl}_{3}\right)$ afforded a purple solid (69 mg, 15\%): mp onset $335{ }^{\circ} \mathrm{C} ;{ }^{1} \mathrm{H}$ NMR $\delta 2.73(\mathrm{~s}, 3 \mathrm{H}), 3.77(\mathrm{~d}, J=6.6$ $\mathrm{Hz}, 2 \mathrm{H}), 4.67$ (s, 6H), 5.29-5.40 (m, 2H), 6.25-6.39 (m, 1H), 7.56-7.60 (m, 4H), 8.07-8.14 (m, 4H), 8.98-9.00 (m, 4H), $9.56(\mathrm{~d}, J=4.5 \mathrm{~Hz}, 4 \mathrm{H})$; LDMS obsd 605.3; FABMS obsd 606.1798, calcd $606.1762\left(\mathrm{C}_{38} \mathrm{H}_{30} \mathrm{~N}_{4} \mathrm{Zn}\right) ; \lambda_{\text {abs }} 425,515,554,597 \mathrm{~nm}$. 
Zn(II)-5-(4-Allylphenyl)-15-mesityl-10,20-dimethylporphyrin (Zn18). Following the procedure for $\mathbf{Z n 1 1}$, the condensation of 35-diol (derived from 35-SnBu $; 427 \mathrm{mg}, 0.740 \mathrm{mmol}$ ) and $30(196 \mathrm{mg}, 0.740 \mathrm{mmol})$ for $30 \mathrm{~min}$, oxidation with DDQ, passage through a silica pad $\left(\mathrm{CH}_{2} \mathrm{Cl}_{2}\right)$, and metalation with $\mathrm{Zn}(\mathrm{OAc})_{2} \cdot 2 \mathrm{H}_{2} \mathrm{O}$ followed by chromatography (silica, $\mathrm{CHCl}_{3}$ ) afforded a purple solid $(90 \mathrm{mg}, 19 \%)$ : mp onset $290{ }^{\circ} \mathrm{C} ;{ }^{1} \mathrm{H}$ NMR $\delta 1.83(\mathrm{~s}, 6 \mathrm{H}), 2.66(\mathrm{~s}, 3 \mathrm{H})$, $3.77(\mathrm{~d}, J=6.6 \mathrm{~Hz}, 2 \mathrm{H}), 4.66(\mathrm{~s}, 6 \mathrm{H}), 5.29-5.40(\mathrm{~m}, 2 \mathrm{H}), 6.25-6.39(\mathrm{~m}, 1 \mathrm{H}), 7.30(\mathrm{~s}, 2 \mathrm{H}), 7.58$ $(\mathrm{d}, J=7.8 \mathrm{~Hz}, 2 \mathrm{H}), 8.11$ (d, $J=7.8 \mathrm{~Hz}, 2 \mathrm{H}), 8.82(\mathrm{~d}, J=4.5 \mathrm{~Hz}, 2 \mathrm{H}), 8.95$ (d, $J=4.5 \mathrm{~Hz}, 2 \mathrm{H})$, $9.53(\mathrm{~d}, J=4.5 \mathrm{~Hz}, 4 \mathrm{H})$; LDMS obsd 634.4; FABMS obsd 634.2120, calcd 634.2075 $\left(\mathrm{C}_{40} \mathrm{H}_{34} \mathrm{~N}_{4} \mathrm{Zn}\right) ; \lambda_{\text {abs }} 424,515,553,597 \mathrm{~nm}$.

Zn(II)-5-(4-Allylphenyl)-10,20-dimethyl-15-(2,4,6-triethylphenyl)porphyrin (Zn19). Following the procedure for $\mathbf{Z n 1 1}$, the condensation of 35-diol (derived from 35-SnBu $\mathbf{H}_{2} ; 427$ $\mathrm{mg}, 0.740 \mathrm{mmol})$ and $31(227 \mathrm{mg}, 0.740 \mathrm{mmol})$ for $25 \mathrm{~min}$, oxidation with DDQ, passage through a silica pad $\left(\mathrm{CH}_{2} \mathrm{Cl}_{2}\right)$, and metalation with $\mathrm{Zn}(\mathrm{OAc})_{2} \cdot 2 \mathrm{H}_{2} \mathrm{O}$ followed by chromatography [silica, $\mathrm{CHCl}_{3} /$ hexanes (1:1)] afforded a purple solid (45 mg, 9\%): mp onset $230{ }^{\circ} \mathrm{C} ;{ }^{1} \mathrm{H}$ NMR $\delta$ 0.71 (t, $J=7.5 \mathrm{~Hz}, 6 \mathrm{H}), 1.56$ (t, $J=7.5 \mathrm{~Hz}, 3 \mathrm{H}) 2.10$ (q. $4 \mathrm{H}), 2.99$ (q, $2 \mathrm{H}), 3.77$ (d, $J=6.6 \mathrm{~Hz}$, $2 \mathrm{H}), 4.66(\mathrm{~s}, 6 \mathrm{H}), 5.29-5.40(\mathrm{~m}, 2 \mathrm{H}), 6.25-6.39(\mathrm{~m}, 1 \mathrm{H}), 7.34(\mathrm{~s}, 2 \mathrm{H}), 7.58(\mathrm{~d}, J=7.8 \mathrm{~Hz}, 2 \mathrm{H})$, $8.11(\mathrm{~d}, J=7.8 \mathrm{~Hz}, 2 \mathrm{H}), 8.82(\mathrm{~d}, J=4.5 \mathrm{~Hz}, 2 \mathrm{H}), 8.95(\mathrm{~d}, J=4.5 \mathrm{~Hz}, 2 \mathrm{H}), 9.50(\mathrm{~d}, J=4.5 \mathrm{~Hz}$, $2 \mathrm{H}), 9.54(\mathrm{~d}, J=4.5 \mathrm{~Hz}, 2 \mathrm{H})$; LDMS obsd 676.4; FABMS obsd 676.2580, calcd 676.2544 $\left(\mathrm{C}_{43} \mathrm{H}_{40} \mathrm{~N}_{4} \mathrm{Zn}\right) ; \lambda_{\text {abs }} 425,515,554,597 \mathrm{~nm}$.

1-Iodo-4-(1,1-dimethoxymethyl)benzene (20). A solution of 4-iodobenzaldehyde (10.0 g, $43.1 \mathrm{mmol})$ in $\mathrm{MeOH}(150 \mathrm{~mL})$ was treated with $\mathrm{TiCl}_{4}(80 \mu \mathrm{L}, 430 \mu \mathrm{mol})$ under argon for 15 min. TEA $(0.2 \mathrm{~mL})$ was added. After $15 \mathrm{~min}$, water and $\mathrm{Et}_{2} \mathrm{O}$ were added. The organic layer was collected, dried $\left(\mathrm{Na}_{2} \mathrm{SO}_{4}\right)$, filtered, and concentrated to give a pale yellow oil $(11.5 \mathrm{~g}, 96 \%)$ : ${ }^{1} \mathrm{H}$ NMR $\delta 3.30(\mathrm{~s}, 6 \mathrm{H}), 5.34(\mathrm{~s}, 1 \mathrm{H}), 7.19(\mathrm{~d}, J=8.4 \mathrm{~Hz}, 2 \mathrm{H}), 7.70(\mathrm{~d}, J=8.4 \mathrm{~Hz}, 2 \mathrm{H}) ;{ }^{13} \mathrm{C}$ NMR $\delta$ 52.4, 94.3, 102.1, 128.6, 137.1, 137.6.

1-(1,1-Dimethoxymethyl)-4-vinylbenzene (21). A sample of 20 (8.50 g, $30.6 \mathrm{mmol})$ was treated with vinylmagnesium bromide $(33.6 \mathrm{~mL}, 33.6 \mathrm{mmol}, 1.0 \mathrm{M}$ solution in THF) followed by $\mathrm{Pd}\left(\mathrm{PPh}_{3}\right)_{2} \mathrm{Cl}_{2}(220 \mathrm{mg}, 1 \mathrm{~mol} \%)$. The mixture was stirred at room temperature under argon for $2 \mathrm{~h}$. Water and $\mathrm{Et}_{2} \mathrm{O}$ were added. The aqueous layer was washed with $\mathrm{Et}_{2} \mathrm{O}$. The organic layer was collected, dried $\left(\mathrm{Na}_{2} \mathrm{SO}_{4}\right)$, filtered, and concentrated. Chromatography [silica, $\mathrm{Et}_{2} \mathrm{O} /$ hexanes/TEA (25:75:1)] afforded a colorless oil $(3.51 \mathrm{~g}, 64 \%):{ }^{1} \mathrm{H}$ NMR $\delta 3.33(\mathrm{~s}$, $6 \mathrm{H}), 5.25(\mathrm{~d}, J=10.0 \mathrm{~Hz}, 1 \mathrm{H}), 5.39(\mathrm{~s}, 1 \mathrm{H}), 5.75(\mathrm{~d}, J=16.8 \mathrm{~Hz}, 1 \mathrm{H}), 6.72\left(\mathrm{dd}, J^{1}=16.8 \mathrm{~Hz}, J^{2}\right.$ $=10.0 \mathrm{~Hz}, 1 \mathrm{H}), 7.41(\mathrm{~s}, 4 \mathrm{H}) ;{ }^{13} \mathrm{C}$ NMR $\delta 52.3,102.6,113.9,125.9,126.5,126.8,128.0,136.3$, 137.4, 137.5; FABMS obsd 178.0944, calcd $178.0994\left(\mathrm{C}_{11} \mathrm{H}_{14} \mathrm{O}_{2}\right)$.

4-Allylbenzaldehyde (23). Following a standard procedure, ${ }^{38}$ a solution of $22(2.36 \mathrm{~g}$, $10.2 \mathrm{mmol})$ in $\mathrm{CH}_{2} \mathrm{Cl}_{2}(60 \mathrm{~mL})$ was treated with TFA $(12 \mathrm{~mL})$ and water $(0.3 \mathrm{~mL})$. The solution was stirred for $18 \mathrm{~h}$. Then aqueous $\mathrm{NaHCO}_{3}(5 \%, 150 \mathrm{~mL})$ was added. The organic phase was washed with aqueous $\mathrm{NaHCO}_{3}$ and brine, then dried $\left(\mathrm{Na}_{2} \mathrm{SO}_{4}\right)$ and concentrated. Chromatography (silica, $\mathrm{CH}_{2} \mathrm{Cl}_{2}$ /hexanes, 1:2) afforded a colorless oil that partially solidified after standing at $0{ }^{\circ} \mathrm{C}$ for a few weeks $(1.20 \mathrm{~g}, 81 \%):{ }^{1} \mathrm{H}$ NMR $\delta 3.47(\mathrm{~d}, J=6.4 \mathrm{~Hz} 2 \mathrm{H}), 5.09$ $5.15(\mathrm{~m}, 2 \mathrm{H}), 5.92-5.99(\mathrm{~m}, 1 \mathrm{H}), 7.36(\mathrm{~d}, J=8.4 \mathrm{~Hz}, 2 \mathrm{H}), 7.82(\mathrm{~d}, J=8.0 \mathrm{~Hz}, 2 \mathrm{H}), 9.81(\mathrm{~s}, 1 \mathrm{H})$; ${ }^{13} \mathrm{C}$ NMR 40.1, 116.7, 129.1, 129.8, 134.5, 135.9, 147.2, 191.7; FABMS obsd 146.0739, calcd $146.0732\left(\mathrm{C}_{10} \mathrm{H}_{10} \mathrm{O}\right)$.

5-Allyldipyrromethane (24). Following a standard procedure, ${ }^{40,41}$ a solution of 3butenal diethylacetal $(4.00 \mathrm{~g}, 27.7 \mathrm{mmol})$ in pyrrole $(194 \mathrm{~mL}, 2.77 \mathrm{~mol})$ at room temperature 
under argon was treated with TFA (467 $\mu \mathrm{L}, 2.77 \mathrm{mmol})$ for $10 \mathrm{~min}$. TEA (221 $\mu \mathrm{L}, 2.77 \mathrm{mmol})$ was added. The mixture was concentrated under high vacuum. The residue was chromatographed [silica, hexanes/ethyl acetate (4:1)] to afford a pale orange oil (1.85 g, 36\%): ${ }^{1} \mathrm{H}$ NMR $\delta 2.86(\mathrm{t}, J=8.0 \mathrm{~Hz}, 2 \mathrm{H}), 4.11(\mathrm{t}, J=8.0 \mathrm{~Hz}, 1 \mathrm{H}), 5.02-5.12(\mathrm{~m}, 2 \mathrm{H}), 5.83(\mathrm{~m}, 1 \mathrm{H})$, $6.08(\mathrm{~s}, 2 \mathrm{H}), 6.15(\mathrm{~m}, 2 \mathrm{H}), 6.65(\mathrm{~m}, 2 \mathrm{H}), 7.83(\mathrm{br} \mathrm{s}, 2 \mathrm{H}) ;{ }^{13} \mathrm{C}$ NMR $\delta 37.3,38.7,105.6,107.6$, 116.3, 117.1, 132.8, 136.2; FABMS obsd 187.1230, calcd 187.1235 [(M+H) $\left.{ }^{+}\right],\left(\mathrm{M}=\mathrm{C}_{12} \mathrm{H}_{14} \mathrm{~N}_{2}\right)$.

5-(3-Butenyl)dipyrromethane (25). Following a standard procedure, ${ }^{42}$ a solution of 4pentenal $(4.00 \mathrm{~g}, 47.6 \mathrm{mmol})$ in pyrrole $(332 \mathrm{~mL}, 4.76 \mathrm{~mol})$ at room temperature under argon was treated with $\mathrm{InCl}_{3}(1.05 \mathrm{~g}, 4.76 \mathrm{mmol})$ for $1.5 \mathrm{~h}$. Powdered $\mathrm{NaOH}(5.71 \mathrm{~g}, 143 \mathrm{mmol})$ was added. After stirring for $30 \mathrm{~min}$, the mixture was suction-filtered. The filtrate was concentrated under high vacuum. The resulting residue was chromatographed [silica, hexanes/ethyl acetate (4:1)] to afford a pale yellow oil $(7.48 \mathrm{~g}, 78 \%):{ }^{1} \mathrm{H}$ NMR $\delta 2.05(\mathrm{~m}, 4 \mathrm{H}), 4.00(\mathrm{~m}, 1 \mathrm{H}), 4.96-$ $5.05(\mathrm{~m}, 2 \mathrm{H}), 5.80(\mathrm{~m}, 1 \mathrm{H}), 6.08(\mathrm{~s}, 2 \mathrm{H}), 6.15(\mathrm{~m}, 2 \mathrm{H}), 6.62(\mathrm{~m}, 2 \mathrm{H}), 7.71(\mathrm{br} \mathrm{s}, 2 \mathrm{H}) ;{ }^{13} \mathrm{C}$ NMR $\delta$ $31.3,33.1,36.5,105.5,107.6,114.9,117.1,133.1$, 138.0; FABMS obsd 200.1321, calcd $200.1313\left(\mathrm{C}_{13} \mathrm{H}_{16} \mathrm{~N}_{2}\right)$.

5-(4-Vinylphenyl)dipyrromethane (27). Following the procedure for $\mathbf{2 5}$, the reaction of $21(2.78 \mathrm{~g}, 15.6 \mathrm{mmol})$ in pyrrole $(108 \mathrm{~mL}, 1.56 \mathrm{~mol})$ and the standard workup including chromatography (silica, $\mathrm{CH}_{2} \mathrm{Cl}_{2}$ followed by silica, toluene) afforded a yellow solid $(2.52 \mathrm{~g}$, 65\%): mp 75-78 ${ }^{\circ} \mathrm{C} ;{ }^{1} \mathrm{H}$ NMR $\oint\left(\mathrm{CD}_{2} \mathrm{Cl}_{2}\right) 5.24(\mathrm{~d}, J=10.0 \mathrm{~Hz}, 1 \mathrm{H}), 5.44(\mathrm{~s}, 1 \mathrm{H}), 5.75(\mathrm{~d}, J=$ $16.8 \mathrm{~Hz}, 1 \mathrm{H}), 5.87-5.88(\mathrm{~m}, 2 \mathrm{H}), 6.11-6.13(\mathrm{~m}, 2 \mathrm{H}), 6.68-6.76(\mathrm{~m}, 3 \mathrm{H}), 7.17(\mathrm{~d}, J=8.0 \mathrm{~Hz}$, $2 \mathrm{H}), 7.38(\mathrm{~d}, J=8.0 \mathrm{~Hz}, 2 \mathrm{H}), 8.01$ (brs, $2 \mathrm{H}) ;{ }^{13} \mathrm{C}$ NMR $\delta\left(\mathrm{CD}_{2} \mathrm{Cl}_{2}\right) 44.2,107.5,108.8,114.1$, 117.8, 126.9, 129.0, 133.0, 136.8, 136.9, 142.6; FABMS obsd 248.1311, calcd 248.1313; Anal. Calcd for $\mathrm{C}_{17} \mathrm{H}_{16} \mathrm{~N}_{2}$ : C, 82.22; H, 6.49; N, 11.28; Found: C, 82.44; H, 6.45; N, 11.24.

5-(4-Allylphenyl)dipyrromethane (28). Following the procedure for 25, the reaction of $23(1.75 \mathrm{~g}, 12.0 \mathrm{mmol})$ in pyrrole $(83 \mathrm{~mL}, 1.2 \mathrm{~mol})$ and the standard workup including chromatography (silica, toluene) followed by recrystallization from $\mathrm{EtOH} / \mathrm{H}_{2} \mathrm{O}(6: 1)$ afforded an off-white solid (1.92 g, 61\%): $\mathrm{mp} 60-62{ }^{\circ} \mathrm{C} ;{ }^{1} \mathrm{H}$ NMR $\delta 3.37(\mathrm{~d}, J=8.0 \mathrm{~Hz}, 2 \mathrm{H}), 5.06-5.11(\mathrm{~m}$, 2H), 5.46 (s, 1H), 5.93-5.99 (m, 3H), 6.15-6.17 (m, 2H), 6.69-6.70 (m, 2H), $7.15(\mathrm{~s}, 4 \mathrm{H}), 7.91$ (brs, 2H); ${ }^{13} \mathrm{C}$ NMR $\delta 39.8,43.6,107.1,108.4,115.9,117.1,128.4,128.8,132.6,137.3,138.8$, 139.8; FABMS obsd 262.1476, calcd 262.1470; Anal. Calcd for $\mathrm{C}_{18} \mathrm{H}_{18} \mathrm{~N}_{2}$ : C, 82.41; H, 6.92; N, 10.68; Found: C, 82.44; H, 6.85; N, 10.44 .

5-(2,4,6-Triethylphenyl)dipyrromethane (31). Following a standard procedure for mesitaldehyde, ${ }^{42}$ a solution of $2,4,6$-triethylbenzaldehyde $(5.70 \mathrm{~g}, 30.0 \mathrm{mmol})$ in pyrrole $(210$ $\mathrm{mL}, 3.00 \mathrm{~mol})$ was treated with $\mathrm{MgBr}_{2}(2.76 \mathrm{~g}, 15.0 \mathrm{mmol})$ at room temperature for $1 \mathrm{~h}$. Powdered $\mathrm{NaOH}(6.0 \mathrm{~g}, 15 \mathrm{mmol})$ was added. After stirring for $30 \mathrm{~min}$, the mixture was suction-filtered. The filtrate was concentrated under high vacuum. Chromatography (silica, $\mathrm{CHCl}_{3}$ followed by silica, toluene) of the residue afforded a brown oil $(5.22 \mathrm{~g}, 57 \%):{ }^{1} \mathrm{H}$ NMR $\delta$ 0.93 (brs, 6H), 1.28 (t, $J=8.0 \mathrm{~Hz}, 3 \mathrm{H}), 2.53$ (brs, 4H), 2.64 (q, $J=7.6 \mathrm{~Hz}, 2 \mathrm{H}), 5.96$ (s, 1H), $6.07(\mathrm{~s}, 2 \mathrm{H}), 6.19(\mathrm{~m}, 2 \mathrm{H}), 6.66(\mathrm{~s}, 2 \mathrm{H}), 6.97(\mathrm{~s}, 2 \mathrm{H}), 7.95(\mathrm{brs}, 2 \mathrm{H}) ;{ }^{13} \mathrm{C}$ NMR $\delta$ 15.2, 27.0, 28.3, 37.3, 106.4, 108.3, 115.9, 127.3, 131.8, 133.1, 143.1; FABMS obsd 306.2103, calcd 306.2096 $\left(\mathrm{C}_{21} \mathrm{H}_{26} \mathrm{~N}_{2}\right)$.

Dibutyl[5,10-dihydro-1,9-di-p-toluoyl-5-p-tolyldipyrrinato]tin(IV)

$\left(32-\mathrm{SnBu}_{2}\right)$. Following a general diacylation procedure, ${ }^{39}$ a solution of $\mathbf{2 9}(5.00 \mathrm{~g}, 21.2 \mathrm{mmol})$ in toluene (125 $\mathrm{mL})$ was treated with $\mathrm{EtMgBr}(100 \mathrm{~mL}, 100 \mathrm{mmol}, 1.0 \mathrm{M}$ in THF) followed by $p$-toluoyl chloride $(7.0 \mathrm{~mL}, 53 \mathrm{mmol})$. After quenching and extractive workup, the crude material was 
dissolved in $\mathrm{CH}_{2} \mathrm{Cl}_{2}(200 \mathrm{~mL})$. Samples of TEA $(8.9 \mathrm{~mL}, 64 \mathrm{mmol})$ and $\mathrm{Bu}_{2} \mathrm{SnCl}_{2}(6.44 \mathrm{~g}, 21.2$ mmol) were added. After $30 \mathrm{~min}$, the solution was concentrated. Column chromatography (silica, $\mathrm{CH}_{2} \mathrm{Cl}_{2}$ ) followed by crystallization $(\mathrm{MeOH})$ afforded a pale green solid $(8.54 \mathrm{~g}, 57 \%)$ : mp 124-126 ${ }^{\circ} \mathrm{C} ;{ }^{1} \mathrm{H}$ NMR $\delta 0.69(\mathrm{t}, J=7.2 \mathrm{~Hz}, 3 \mathrm{H}), 0.74(\mathrm{t}, J=7.2 \mathrm{~Hz}, 3 \mathrm{H}), 1.09-1.14(\mathrm{~m}, 2 \mathrm{H})$, 1.19-1.25 (m, 2H), 1.30-1.34 (m, 2H), 1.41-1.45 (m, 2H), 1.47-1.52 (m, 2H), 1.66-1.70 (m, $2 \mathrm{H}), 2.31(\mathrm{~s}, 3 \mathrm{H}), 2.44(\mathrm{~s}, 6 \mathrm{H}), 5.56(\mathrm{~s}, 1 \mathrm{H}), 6.19(\mathrm{~d}, J=4.0 \mathrm{~Hz}, 2 \mathrm{H}), 7.08-7.11(\mathrm{~m}, 6 \mathrm{H}), 7.29(\mathrm{~d}$, $J=8.0 \mathrm{~Hz}, 4 \mathrm{H}), 7.82(\mathrm{~d}, J=8.0 \mathrm{~Hz}, 4 \mathrm{H}) ;{ }^{13} \mathrm{C} \mathrm{NMR} \delta 13.55,13.57,21.0,21.5,23.9,24.7,25.9$, 26.3, 27.16, 27.22, 45,2, 115.0, 123.7, 127.9, 129.0, 129.1, 129.3, 135.0, 135.7, 136.2, 141.3, 142.0, 151.7, 184.3; FABMS obsd 705.2503, calcd 705.2525 [(M+H) ${ }^{+}$; Anal. Calcd for $\mathrm{C}_{40} \mathrm{H}_{44} \mathrm{~N}_{2} \mathrm{O}_{2} \mathrm{Sn} ; \mathrm{C}, 68.29 ; \mathrm{H}, 6.30$; N, 3.98; Found: C, 68.33; H, 6.35; N, 3.92.

Dibutyl[5,10-dihydro-5-mesityl-1,9-di-p-toluoyldipyrrinato]tin(IV)

$\left(33-\mathrm{SnBu}_{2}\right)$.

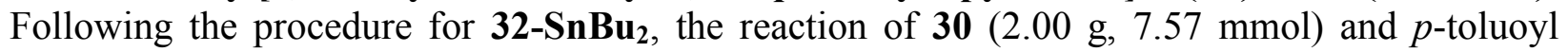
chloride $(2.00 \mathrm{~mL}, 15.1 \mathrm{mmol})$ followed by tin complexation (TEA, $3.2 \mathrm{~mL}, 23 \mathrm{mmol}$; $\mathrm{Bu}_{2} \mathrm{SnCl}_{2}, 2.30 \mathrm{~g}, 7.57 \mathrm{mmol}$ ), chromatography (silica, $\mathrm{CH}_{2} \mathrm{Cl}_{2}$ ), and precipitation (diethyl ether/MeOH) afforded a pale yellow solid $(2.87 \mathrm{~g}, 52 \%)$ : $\mathrm{mp} 151-153{ }^{\circ} \mathrm{C} ;{ }^{1} \mathrm{H}$ NMR $\delta 0.71(\mathrm{t}, J=$ $7.2 \mathrm{~Hz}, 3 \mathrm{H}), 0.78(\mathrm{t}, J=7.2 \mathrm{~Hz}, 3 \mathrm{H}), 1.11-1.21(\mathrm{~m}, 2 \mathrm{H}), 1.21-1.36(\mathrm{~m}, 4 \mathrm{H}), 1.43-1.55(\mathrm{~m}, 4 \mathrm{H})$, $1.70-1.78(\mathrm{~m}, 5 \mathrm{H}), 2.32(\mathrm{~s}, 3 \mathrm{H}), 2.43(\mathrm{~s}, 6 \mathrm{H}), 2.51(\mathrm{~s}, 3 \mathrm{H}), 5.81(\mathrm{~d}, J=4.0 \mathrm{~Hz}, 2 \mathrm{H}), 5.93(\mathrm{~s}, 1 \mathrm{H})$, $6.81(\mathrm{~s}, 1 \mathrm{H}), 6.98(\mathrm{~s}, 1 \mathrm{H}), 7.04(\mathrm{~d}, J=4.0 \mathrm{~Hz}, 2 \mathrm{H}), 7.29(\mathrm{~d}, J=8.0 \mathrm{~Hz}, 4 \mathrm{H}), 7.81(\mathrm{~d}, J=8.0 \mathrm{~Hz}$, $4 \mathrm{H}) ;{ }^{13} \mathrm{C}$ NMR $\delta 13.6,20.3,20.7,20.9,21.6,23.5,24.9,26.2,26.4,27.5,40.0,113.9,123.8$, 128.7, 129.0, 130.7, 135.1, 135.3, 136.0, 136.3, 136.4, 138.1, 141.9, 151.9, 183.9; FABMS obsd 733.2826, calcd 733.2816 [(M+H) $\left.{ }^{+}\right]$; Anal. calcd for $\mathrm{C}_{42} \mathrm{H}_{48} \mathrm{~N}_{2} \mathrm{O}_{2} \mathrm{Sn} ; \mathrm{C}, 68.96 ; \mathrm{H}, 6.61 ; \mathrm{N}, 3.83$; Found: C, 68.84; H, 6.61; N, 3.76.

\section{Dibutyl[5,10-dihydro-5-(2,4,6-triethylphenyl)-1,9-di-p-toluoyldipyrrinato]tin(IV)}

$\left(\mathbf{3 4 - S n B u _ { 2 } ) \text { . Following the procedure for 32-SnBu}}\right.$, the reaction of $\mathbf{3 1}$ (2.00 g, $\left.6.52 \mathrm{mmol}\right)$ and p-toluoyl chloride $(2.16 \mathrm{~mL}, 16.3 \mathrm{mmol})$ followed by tin complexation (TEA, $2.7 \mathrm{~mL}, 20 \mathrm{mmol}$; $\mathrm{Bu}_{2} \mathrm{SnCl}_{2}, 1.98 \mathrm{~g}, 6.52 \mathrm{mmol}$ ), chromatography (silica, $\mathrm{CH}_{2} \mathrm{Cl}_{2}$ ), and precipitation (diethyl ether/MeOH) afforded a pink solid (1.96 g, 39\%): mp 136-138 ${ }^{\circ} \mathrm{C} ;{ }^{1} \mathrm{H}$ NMR $\delta 0.71-0.79$ (m, 9H), 1.18-1.30 (m, 12H), 1.47-1.56 (m, 4H), $1.74(\mathrm{~m}, 2 \mathrm{H}), 2.08(\mathrm{q}, J=8.0 \mathrm{~Hz}, 2 \mathrm{H}), 2.43(\mathrm{~s}$, $6 \mathrm{H}), 2.66(\mathrm{q}, J=8.0 \mathrm{~Hz}, 2 \mathrm{H}), 2.81(\mathrm{q}, J=2 \mathrm{H}), 5.81(\mathrm{~d}, J=4.0 \mathrm{~Hz}, 2 \mathrm{H}), 5.88(\mathrm{~s}, 1 \mathrm{H}), 6.93(\mathrm{~s}$, $1 \mathrm{H}), 6.98(\mathrm{~s}, 1 \mathrm{H}), 7.01(\mathrm{~d}, J=4.0 \mathrm{~Hz}, 2 \mathrm{H}), 7.29(\mathrm{~d}, J=8.0 \mathrm{~Hz}, 4 \mathrm{H}), 7.81(\mathrm{~d}, J=8.0 \mathrm{~Hz}, 4 \mathrm{H}) ;{ }^{13} \mathrm{C}$ NMR $\delta 13.7,13.8,15.3,16.7,21.6,23.5,24.8,25.3,26.2,26.5,27.4,27.5,28.1,28.5,39.4$, $114.7,123.6,125.9,127.1,129.01,129.04,135.2,135.3,135.4,141.9,142.1,142.9,143.9$, 152.8, 183.9; FABMS obsd 775.3292, calcd 775.3286 [(M+H) $\left.{ }^{+}\right]$; Anal. calcd for $\mathrm{C}_{45} \mathrm{H}_{54} \mathrm{~N}_{2} \mathrm{O}_{2} \mathrm{Sn}$; C, 69.86; H, 7.04; N, 3.62; Found: C, 69.96; H, 7.00; N, 3.58.

Dibutyl[1,9-diacetyl-5-(4-allylphenyl)-5,10-dihydrodipyrrinato]tin(IV) (35-SnBu $)$. Following the procedure for $\mathbf{3 2 - S n B u}$, the reaction of 28 (1.40 g, $5.34 \mathrm{mmol})$ and acetyl bromide $(0.992 \mathrm{~mL}, 13.4 \mathrm{mmol})$ followed by tin complexation (TEA, $2.2 \mathrm{~mL}, 16 \mathrm{mmol}$; $\mathrm{Bu}_{2} \mathrm{SnCl}_{2}, 1.62 \mathrm{~g}, 5.34 \mathrm{mmol}$ ), chromatography (silica, $\mathrm{CH}_{2} \mathrm{Cl}_{2}$ ), and precipitation (diethyl ether $/ \mathrm{MeOH})$ afforded a pale yellow solid $(1.50 \mathrm{~g}, 57 \%)$ : $\mathrm{mp} 54-56{ }^{\circ} \mathrm{C} ;{ }^{1} \mathrm{H}$ NMR $\delta 0.70(\mathrm{t}, J=$ $7.6 \mathrm{~Hz}, 3 \mathrm{H}), 0.75(\mathrm{t}, J=7.6 \mathrm{~Hz}, 3 \mathrm{H}), 1.05-1.57(\mathrm{~m}, 12 \mathrm{H}), 2.41(\mathrm{~s}, 6 \mathrm{H}), 3.31(\mathrm{~d}, J=6.8 \mathrm{~Hz}, 2 \mathrm{H})$, 5.02-5.07 (m, 2H), $5.48(\mathrm{~s}, 1 \mathrm{H}), 5.88-5.95(\mathrm{~m}, 1 \mathrm{H}), 6.10(\mathrm{~d}, J=4.0 \mathrm{~Hz}, 2 \mathrm{H}), 7.02-7.07(\mathrm{~m}, 6 \mathrm{H})$; ${ }^{13} \mathrm{C}$ NMR $\delta 13.49,13.52,23.2,23.4,24.2,25.9,26.3,27.1,39.7,44.8,114.2,115.7,120.8$, 127.8, 128.0, 128.7, 136.6, 137.3, 138.2, 142.2, 150.7, 188.3; FABMS obsd 579.2036, calcd 579.2034 [(M $\left.+\mathrm{H})^{+}\right]$; Anal. calcd for $\mathrm{C}_{30} \mathrm{H}_{38} \mathrm{~N}_{2} \mathrm{O}_{2} \mathrm{Sn}$; C, 62.41; H, 6.63; N, 4.85; Found: C, $62.46 ; \mathrm{H}, 6.67 ; \mathrm{N}, 4.86$. 
5,10,15-Tri-p-tolylporphyrin (37). Following the procedure for $\mathbf{Z n 1 1}$, the condensation of 32-diol (derived from 32, $1.42 \mathrm{~g}, 3.00 \mathrm{mmol}$ ) and $36(440 \mathrm{mg}, 3.01 \mathrm{mmol}$ ) for $20 \mathrm{~min}$, oxidation with DDQ, addition of TEA $(3 \mathrm{~mL})$, and chromatography [silica, hexanes $/ \mathrm{CH}_{2} \mathrm{Cl}_{2}$, (1:2)] afforded a purple solid (477 mg, 27\%): ${ }^{1} \mathrm{H}$ NMR $\delta-2.97$ (s, 2H), 2.70-2.75 (br s, 9H), $7.56(\mathrm{~d}, J=8.0 \mathrm{~Hz}, 2 \mathrm{H}), 7.60(\mathrm{~d}, J=8.0 \mathrm{~Hz}, 4 \mathrm{H}), 8.11(\mathrm{~d}, J=8.0 \mathrm{~Hz}, 2 \mathrm{H}), 8.14(\mathrm{~d}, J=8.0 \mathrm{~Hz}$, 4H), 8.89-8.95 (m, 4H), 9.05 (d, $J=4.4 \mathrm{~Hz}, 2 \mathrm{H}), 9.33$ (d, $J=4.4 \mathrm{~Hz}, 2 \mathrm{H}), 10.20$ (s, 1H); LDMS obsd 580.4; FABMS obsd 580.2635, calcd $580.2627\left(\mathrm{C}_{41} \mathrm{H}_{32} \mathrm{~N}_{4}\right) ; \lambda_{\text {abs }} 414,509,544,585,641$ nm.

3,7-Dibromo-1,9-bis(3,5-di-tert-butylbenzoyl)dipyrromethane (39). A solution of 38 (1.00 g, $1.73 \mathrm{mmol})$ in $\mathrm{CHCl}_{3}(25 \mathrm{~mL})$ was treated dropwise with $\mathrm{Br}_{2}(177 \mu \mathrm{L}, 3.46 \mathrm{mmol})$ at 0 ${ }^{\circ} \mathrm{C}$ and stirred for $10 \mathrm{~min}$. Water $(25 \mathrm{~mL})$ was added. The organic layer was washed with saturated aqueous $\mathrm{NaHCO}_{3}$, dried $\left(\mathrm{Na}_{2} \mathrm{SO}_{4}\right)$, and filtered. The filtrated was concentrated and the resulting residue was chromatographed [silica, $\mathrm{CH}_{2} \mathrm{Cl}_{2}$ /ethyl acetate (95:5)] affording a beige solid (686 mg, 54\%): mp $250{ }^{\circ} \mathrm{C}$ (dec.); ${ }^{1} \mathrm{H}$ NMR $\delta 1.31$ (s, 36H), $4.25(\mathrm{~s}, 2 \mathrm{H}), 6.67(\mathrm{~m}, 2 \mathrm{H})$, 7.59-7.62 (m, 6H), 11.37 (brs, 2H); ${ }^{13} \mathrm{C}$ NMR $\oint 24.1,31.4,34.9,98.6,121.8,123.9,126.4,130.4$, 134.1, 136.9, 150.7, 185.3; FABMS obsd 735.2161, calcd 735.2147 [(M+ H) ${ }^{+}$; Anal. Calcd. for $\mathrm{C}_{39} \mathrm{H}_{48} \mathrm{BrN}_{2} \mathrm{O}_{2}$ : C, 63.59; H, 6.57; Br, 21.69; Found: C, 63.25; H, 6.46; Br, 21.33.

Zn(II)-3,7-Dibromo-10,20-bis(3,5-di-tert-butylphenyl)-15-mesitylporphyrin (Zn40). Following the procedure for Zn11, the condensation of 39-diol (derived from 39; $470 \mathrm{mg}$, 638 $\mu \mathrm{mol})$ and $30(169 \mathrm{mg}, 638 \mu \mathrm{mol})$ for $15 \mathrm{~min}$, oxidation with DDQ, silica pad filtration $\left(\mathrm{CH}_{2} \mathrm{Cl}_{2}\right)$, and metalation with $\mathrm{Zn}(\mathrm{OAc})_{2} \cdot 2 \mathrm{H}_{2} \mathrm{O}$ followed by chromatography ( silica, $\left.\mathrm{CHCl}_{3}\right)$ afforded a red solid (249 mg, 38\%): $\mathrm{mp}>300{ }^{\circ} \mathrm{C} ;{ }^{1} \mathrm{H}$ NMR $\delta 1.55(\mathrm{~s}, 36 \mathrm{H}), 1.83(\mathrm{~s}, 6 \mathrm{H}), 2.62(\mathrm{~s}$, $3 \mathrm{H}), 7.82(\mathrm{~s}, 2 \mathrm{H}), 8.01(\mathrm{~s}, 4 \mathrm{H}), 8.78(\mathrm{~d}, J=4.5 \mathrm{~Hz}, 2 \mathrm{H}), 8.91(\mathrm{~d}, J=4.5 \mathrm{~Hz}, 2 \mathrm{H}), 9.09$ (s, 2H), $10.42(\mathrm{~s}, 1 \mathrm{H})$, (the Ar-H from the mesityl group is buried under the solvent peak); LDMS obsd 1023.2; FABMS obsd 1022.2463, calcd $1022.2476\left(\mathrm{C}_{57} \mathrm{H}_{60} \mathrm{Br}_{2} \mathrm{~N}_{4} \mathrm{Zn}\right) ; \lambda_{\text {abs }} 426,511,550,585$ nm. (Reaction at a $330 \mu \mathrm{mol}$ scale using $\mathrm{Yb}(\mathrm{OTf})_{3}$ yielded $157 \mathrm{mg}(46 \%)$ of $\mathbf{Z n 3 0}$.)

Zn(II)-10,20-Bis(3,5-di-tert-butylphenyl)-15-mesityl-3,7-divinylporphyrin (Zn41). A solution of $\mathbf{Z n 4 0}(100 \mathrm{mg}, 97 \mu \mathrm{mol})$ in THF $(40 \mathrm{~mL})$ was treated with $\mathrm{Pd}\left(\mathrm{PPh}_{3}\right)_{4}(22 \mathrm{mg}, 19$ $\mu \mathrm{mol})$ and tributyl(vinyl)tin $(618 \mu \mathrm{L}, 1.95 \mathrm{~mol})$ at $60{ }^{\circ} \mathrm{C}$ under argon for $12 \mathrm{~h}$. Water and $\mathrm{CHCl}_{3}$ were added to the mixture. The organic layer was dried and concentrated. Chromatography [silica, $\mathrm{CH}_{2} \mathrm{Cl}_{2} /$ hexanes (3:7)] afforded a purple solid: mp onset $310{ }^{\circ} \mathrm{C} ;{ }^{1} \mathrm{H} \mathrm{NMR} \delta 1.56(\mathrm{~s}$, $36 \mathrm{H}), 1.86(\mathrm{~s}, 6 \mathrm{H}), 2.63(\mathrm{~s}, 3 \mathrm{H}), 5.93(\mathrm{~d}, J=11.4 \mathrm{~Hz}, 2 \mathrm{H}), 6.46(\mathrm{~d}, J=17.6 \mathrm{~Hz}, 2 \mathrm{H}), 7.28$ (s, 2H), $7.81(\mathrm{~s}, 2 \mathrm{H}), 8.12(\mathrm{~s}, 4 \mathrm{H}), 8.31-8.38(\mathrm{~m}, 2 \mathrm{H}), 8.78(\mathrm{~d}, J=4.4 \mathrm{~Hz}, 2 \mathrm{H}), 8.94(\mathrm{~d}, J=4.4 \mathrm{~Hz}$, 2H), $9.14(\mathrm{~s}, 2 \mathrm{H}), 10.30(\mathrm{~s}, 1 \mathrm{H})$; LDMS obsd 920.0; FABMS obsd 918.4523, calcd 918.4579 $\left(\mathrm{C}_{61} \mathrm{H}_{66} \mathrm{~N}_{4} \mathrm{Zn}\right) ; \lambda_{\text {abs }} 436,557 \mathrm{~nm}$.

3,4-Dibromo-2,5-bis(bromomethyl)thiophene (42). A solution of 2,5dimethylthiophene $(5.91 \mathrm{~g}, 52.7 \mathrm{mmol})$ in $\mathrm{CH}_{2} \mathrm{Cl}_{2}(200 \mathrm{~mL})$ was treated dropwise with $\mathrm{Br}_{2}(18.9$ $\mathrm{mL}, 370 \mathrm{mmol}$ ) over $30 \mathrm{~min}$. The resulting dark mixture was stirred at room temperature under argon for $18 \mathrm{~h}$. The mixture was washed with aqueous $\mathrm{NaHSO}_{3}$. The organic layer was washed with water, dried $\left(\mathrm{Na}_{2} \mathrm{SO}_{4}\right)$, and filtered. The filtrate was concentrated to a pale yellow solid. Recrystallization $\left(\mathrm{EtOH} / \mathrm{CHCl}_{3}, 9: 1\right)$ afforded a fluffy white solid $(19.8 \mathrm{~g}, 88 \%)$ : $\mathrm{mp} 98-101{ }^{\circ} \mathrm{C}$; ${ }^{1} \mathrm{H}$ NMR $\delta 4.65$ (s, 4H); ${ }^{13} \mathrm{C}$ NMR $\delta 25.3,116.0$, 135.7; FABMS obsd 423.6785, calcd 423.6767; Anal. Calcd for $\mathrm{C}_{6} \mathrm{H}_{4} \mathrm{Br}_{4} \mathrm{~S}$ : C, 16.85; $\mathrm{H}$ 0.94; Br, 74.72; Found: C, 16.55; H, 0.88; Br, 75.06.

3,4-Dibromo-2,5-bis(pyrrol-2-ylmethyl)thiophene (43). Following a standard procedure $^{40}$ but without added acid, a solution of $42(2.00 \mathrm{~g}, 4.68 \mathrm{mmol})$ in pyrrole $(33 \mathrm{~mL})$ was 
stirred at room temperature under argon for $15 \mathrm{~min}$. A solution of $1 \mathrm{M} \mathrm{NaOH}$ was added followed by $\mathrm{CH}_{2} \mathrm{Cl}_{2}$. The organic layer was concentrated. Chromatography (silica, $\mathrm{CH}_{2} \mathrm{Cl}_{2}$ ) followed by twice recrystallization $\left(\mathrm{EtOH} / \mathrm{H}_{2} \mathrm{O}, 9: 1\right)$ afforded a white solid: $\mathrm{mp} 99-102{ }^{\circ} \mathrm{C} ;{ }^{1} \mathrm{H}$ NMR $\delta 4.09$ (s, 4H), 6.04 (s, 2H), 6.12-6.15 (m, 2H), 6.67-6.69 (m, 2H), 8.01 (brs, 2H); ${ }^{13} \mathrm{C}$ NMR $\delta$ 28.8, 107.1, 108.4, 111.2, 117.6, 127.7, 136.6; FABMS obsd 397.9076, calcd 397.9088; Anal. calcd for $\mathrm{C}_{14} \mathrm{H}_{12} \mathrm{Br}_{2} \mathrm{~N}_{2} \mathrm{~S}$ : C, 42.02; H, 3.02; $\mathrm{Br}, 39.94$; Found: C, 42.12; H, 2.95; $\mathrm{Br}, 40.00$.

2,3-Dibromo-5,10-di-p-tolyl-23H-21-thiaporphyrin (45). A solution of $44(500 \mathrm{mg}$, $1.65 \mathrm{mmol})$ was reduced following a general procedure ${ }^{39}(1.25 \mathrm{~g}, 33.0 \mathrm{mmol})$ and the resulting 44-diol was condensed with $43(660 \mathrm{mg}, 1.65 \mathrm{mmol})$ in $\mathrm{CH}_{2} \mathrm{Cl}_{2}(660 \mathrm{~mL})$ containing $\mathrm{InCl}_{3}$ (47 $\mathrm{mg}, 210 \mu \mathrm{mol})$ at room temperature for $15 \mathrm{~min}$. DDQ $(1.12 \mathrm{~g}, 4.95 \mathrm{mmol})$ was added and the reaction mixture was passed over a silica column $\left(\mathrm{CH}_{2} \mathrm{Cl}_{2}\right)$ to afford a gray solid (265 $\mathrm{mg}, 24 \%$ ): $\mathrm{mp}>450{ }^{\circ} \mathrm{C} ;{ }^{1} \mathrm{H}$ NMR $\delta-2.91$ (brs, $\left.1 \mathrm{H}\right), 2.71$ (s, 6H), 7.56 (s, $\left.J=7.5 \mathrm{~Hz}, 4 \mathrm{H}\right), 8.05$ (d, $J=7.5$ $\mathrm{Hz}, 4 \mathrm{H}), 8.70(\mathrm{~d}, J=4.5 \mathrm{~Hz}, 2 \mathrm{H}), 8.96(\mathrm{~m}, 2 \mathrm{H}), 9.02$ (d, $J=4.5 \mathrm{~Hz}, 2 \mathrm{H}), 10.67$ (s, 2H); LDMS obsd, 665.0; FABMS obsd 664.0058, calcd 662.9979 [(M+H) $\left.{ }^{+}\right],\left(\mathrm{M}=\mathrm{C}_{34} \mathrm{H}_{23} \mathrm{Br}_{2} \mathrm{~N}_{3} \mathrm{~S}\right) ; \lambda_{\text {abs }} 426$, $509,607,670 \mathrm{~nm}$. 


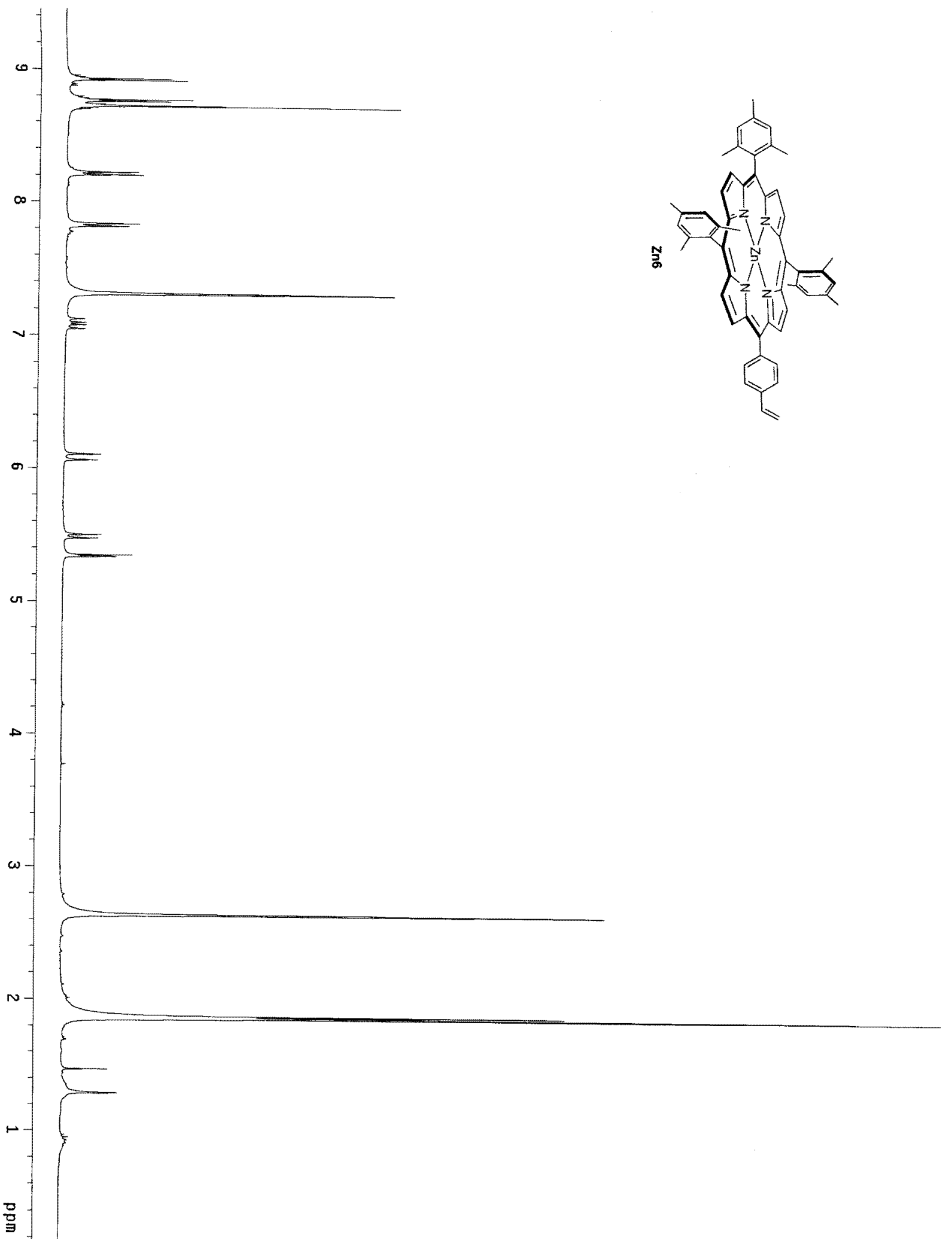




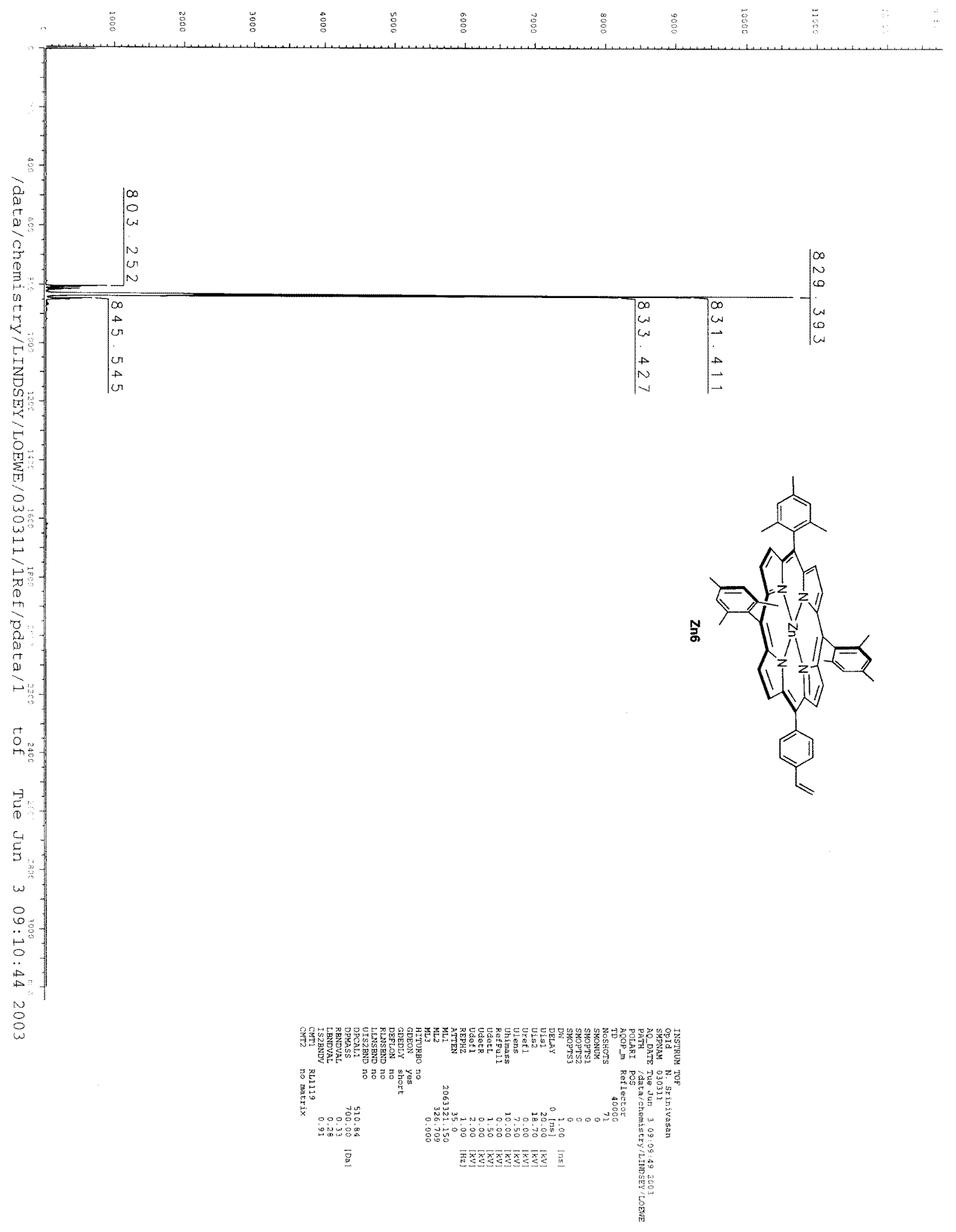

S-11 


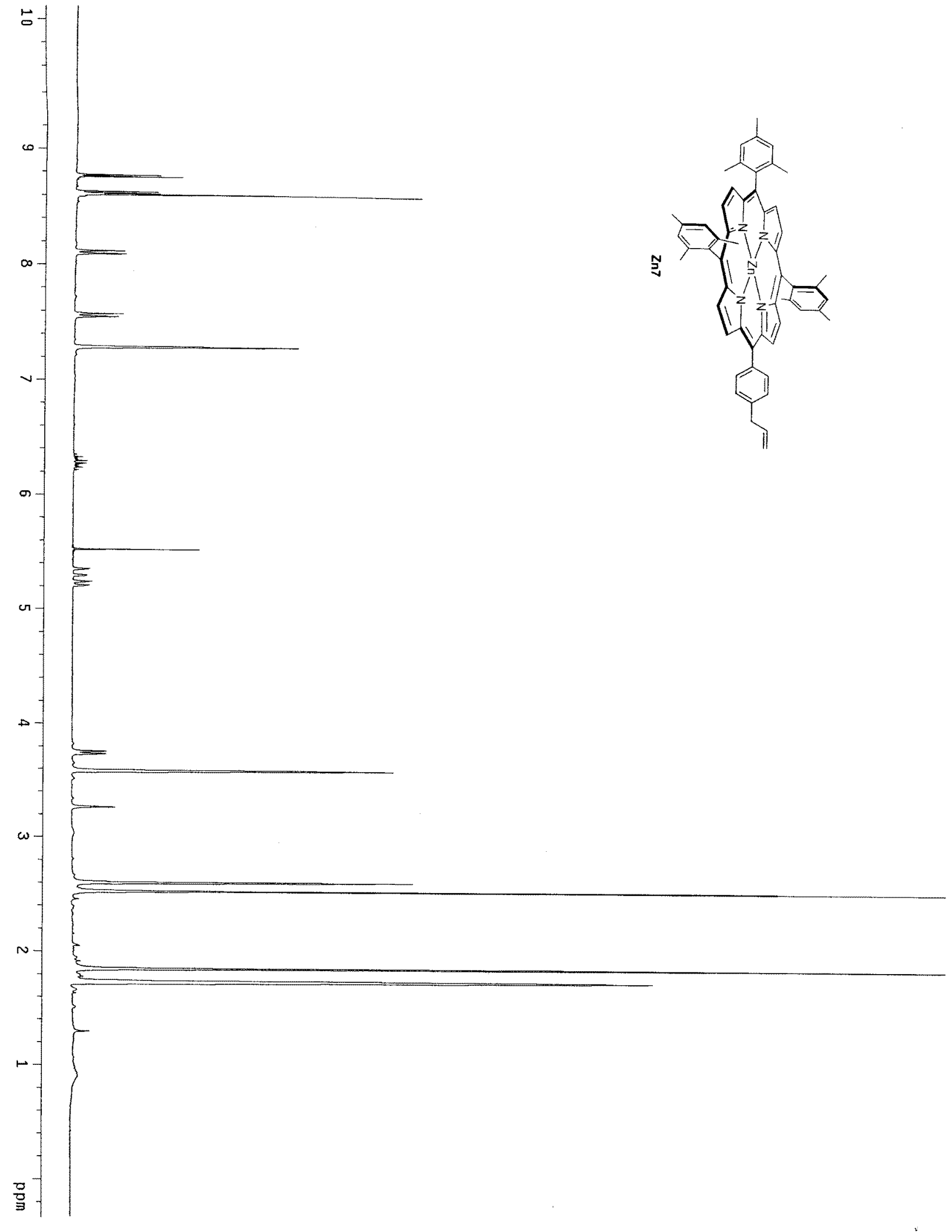

S-12 


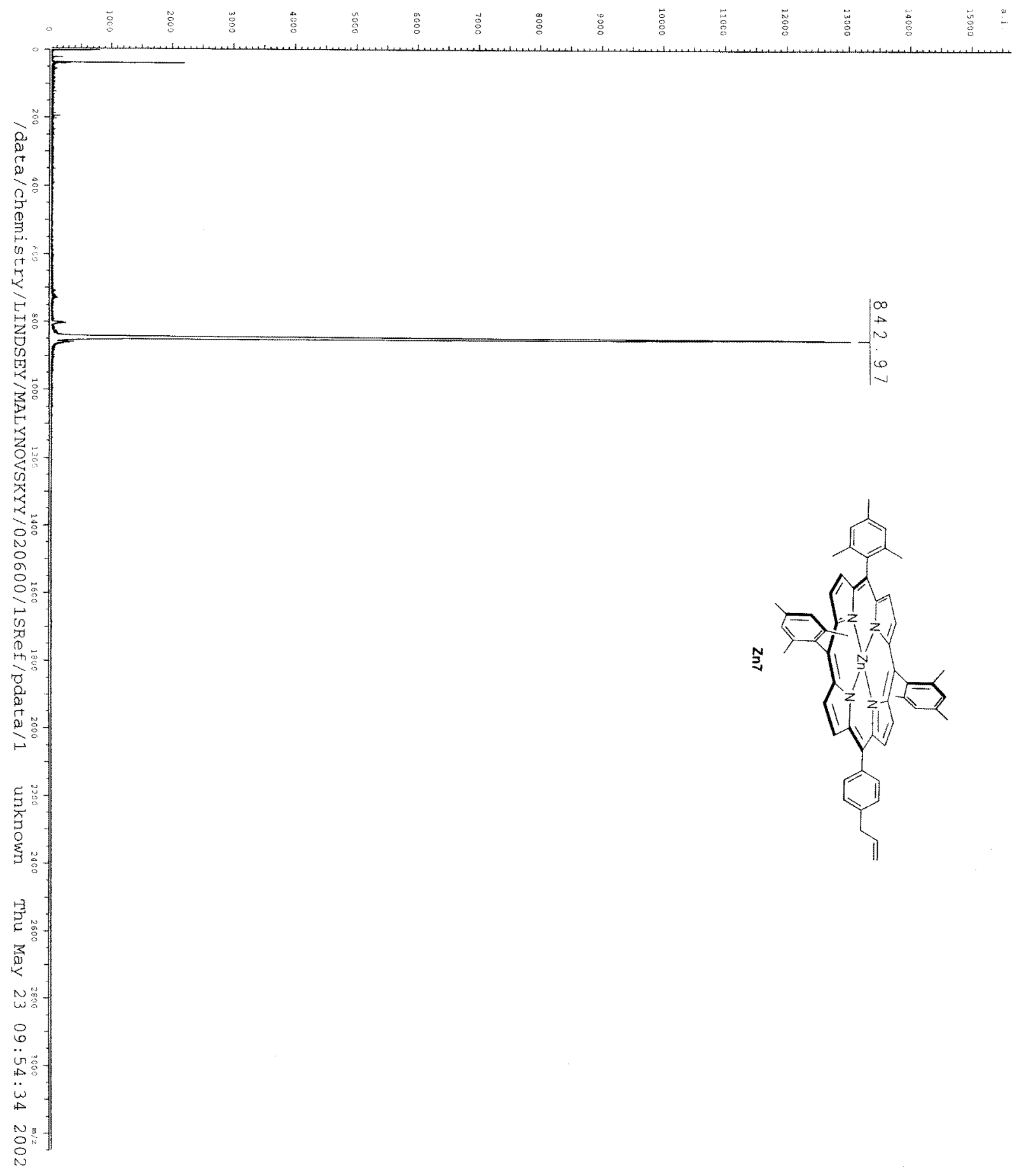

S-13 


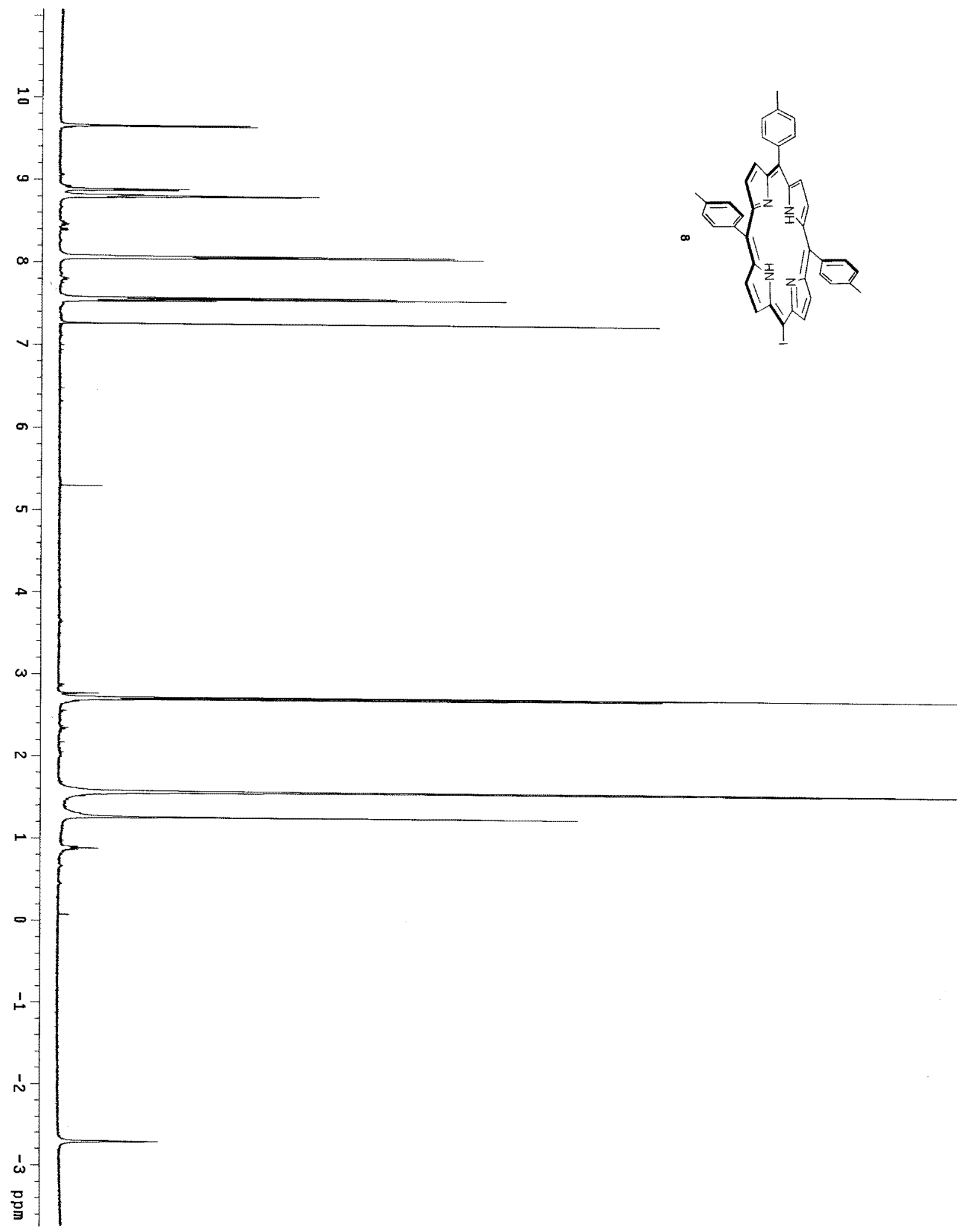




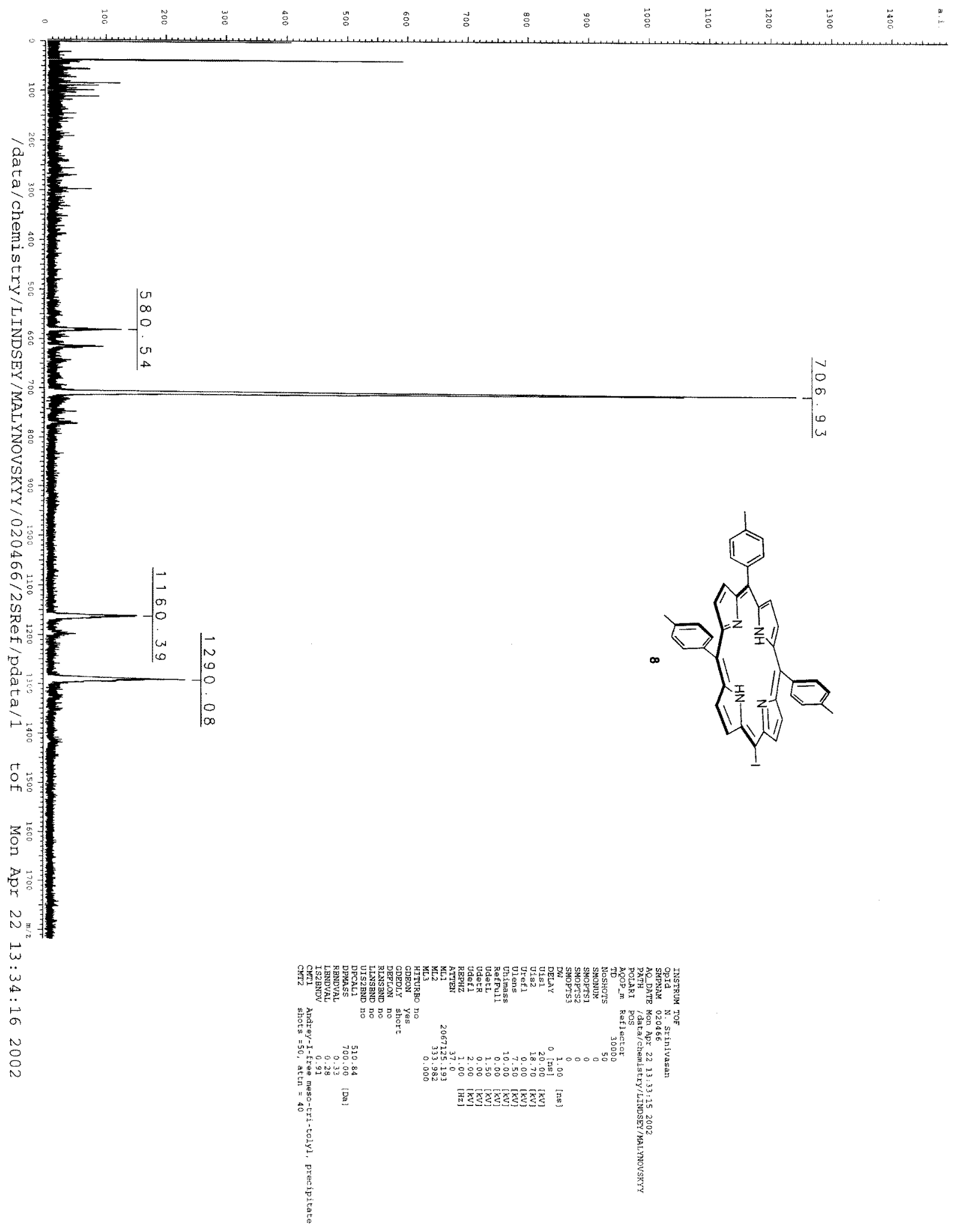




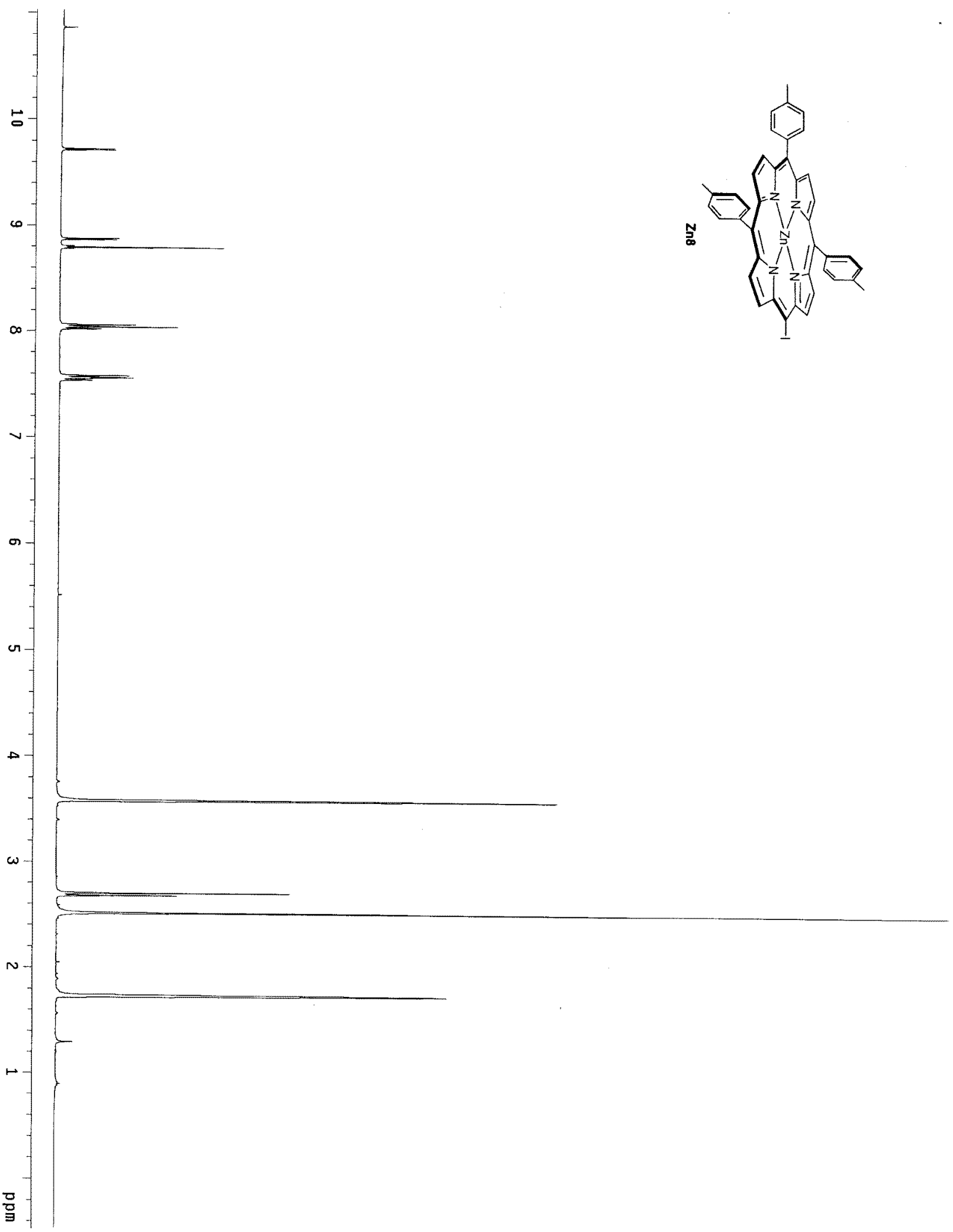

S-16 


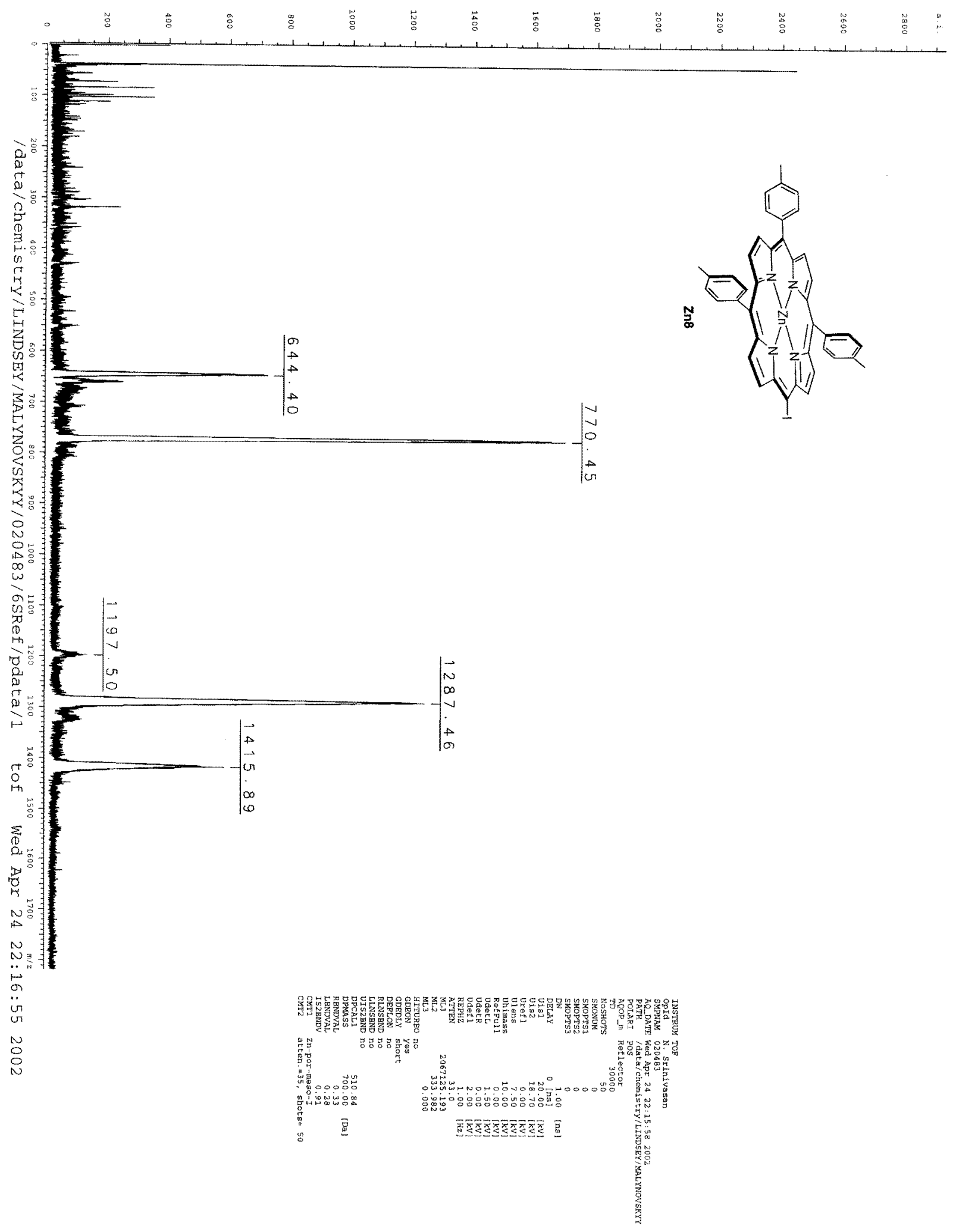




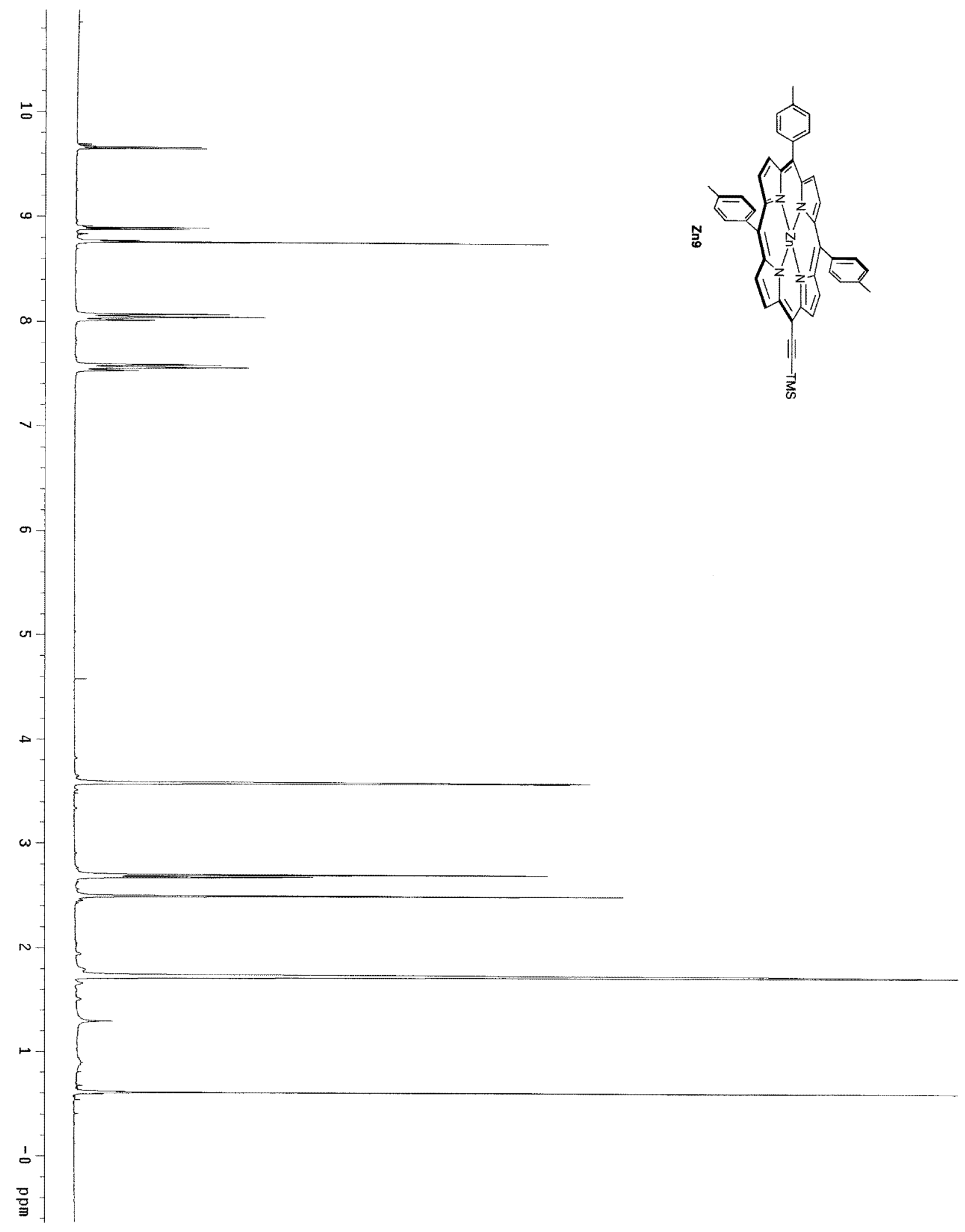




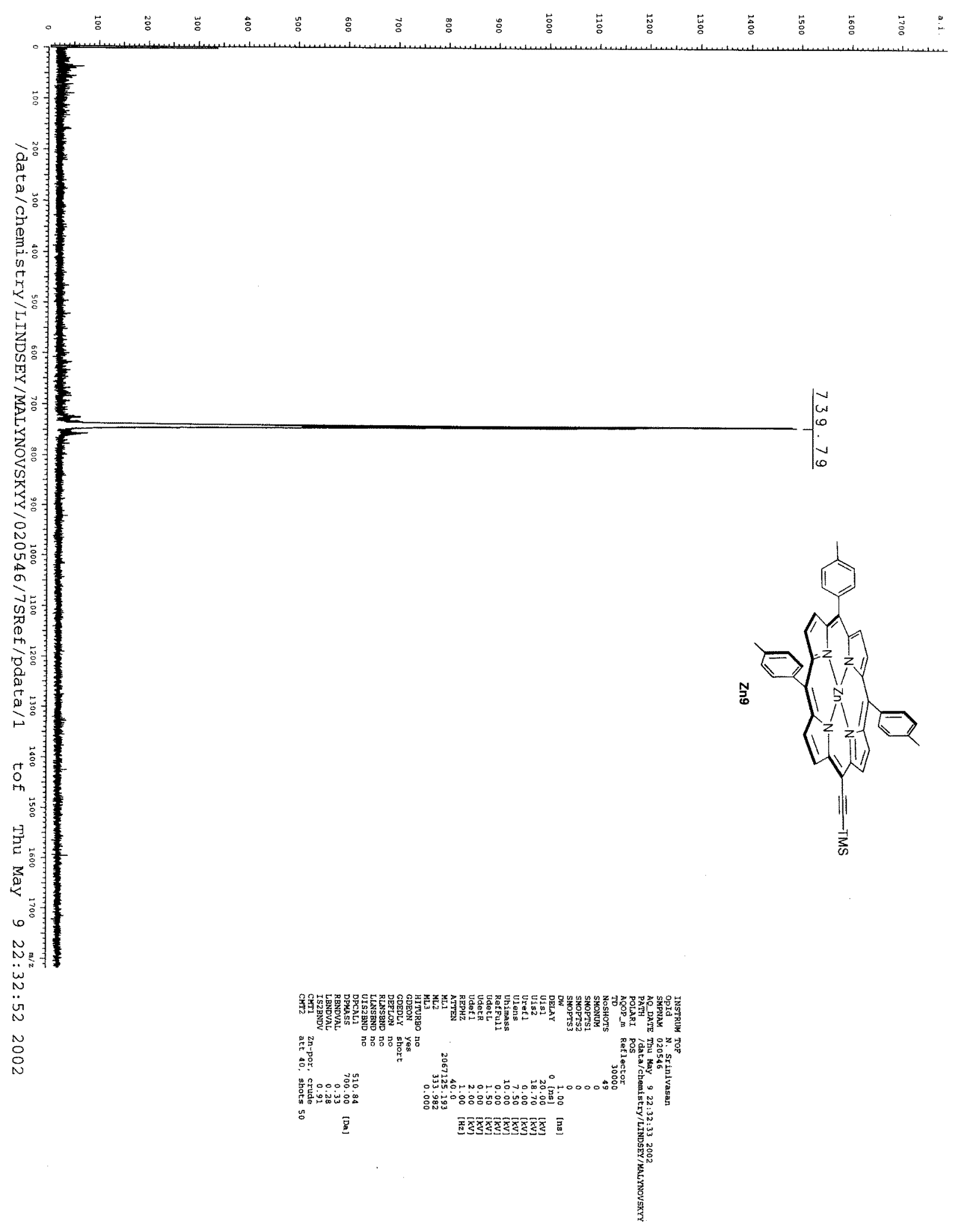

S-19 


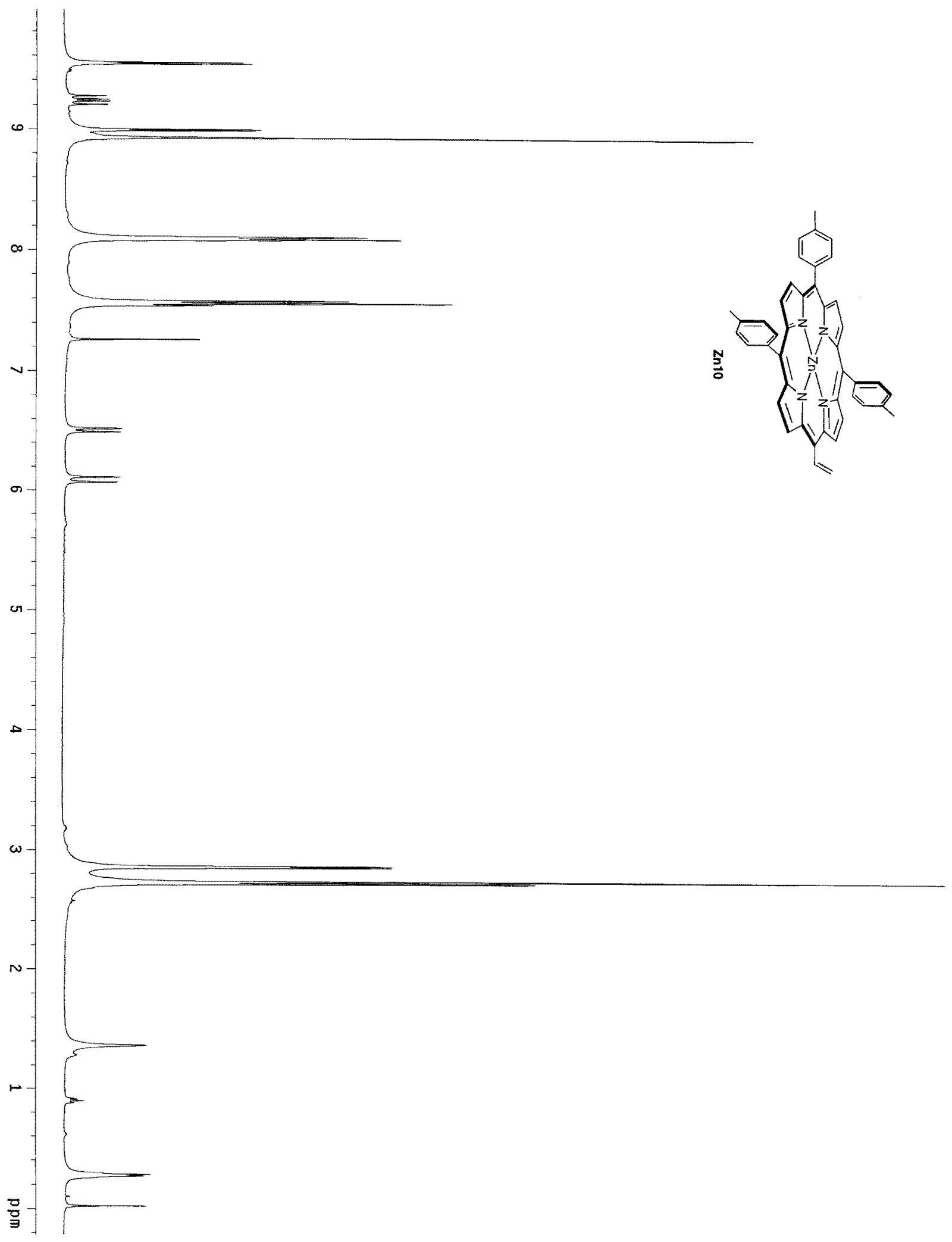




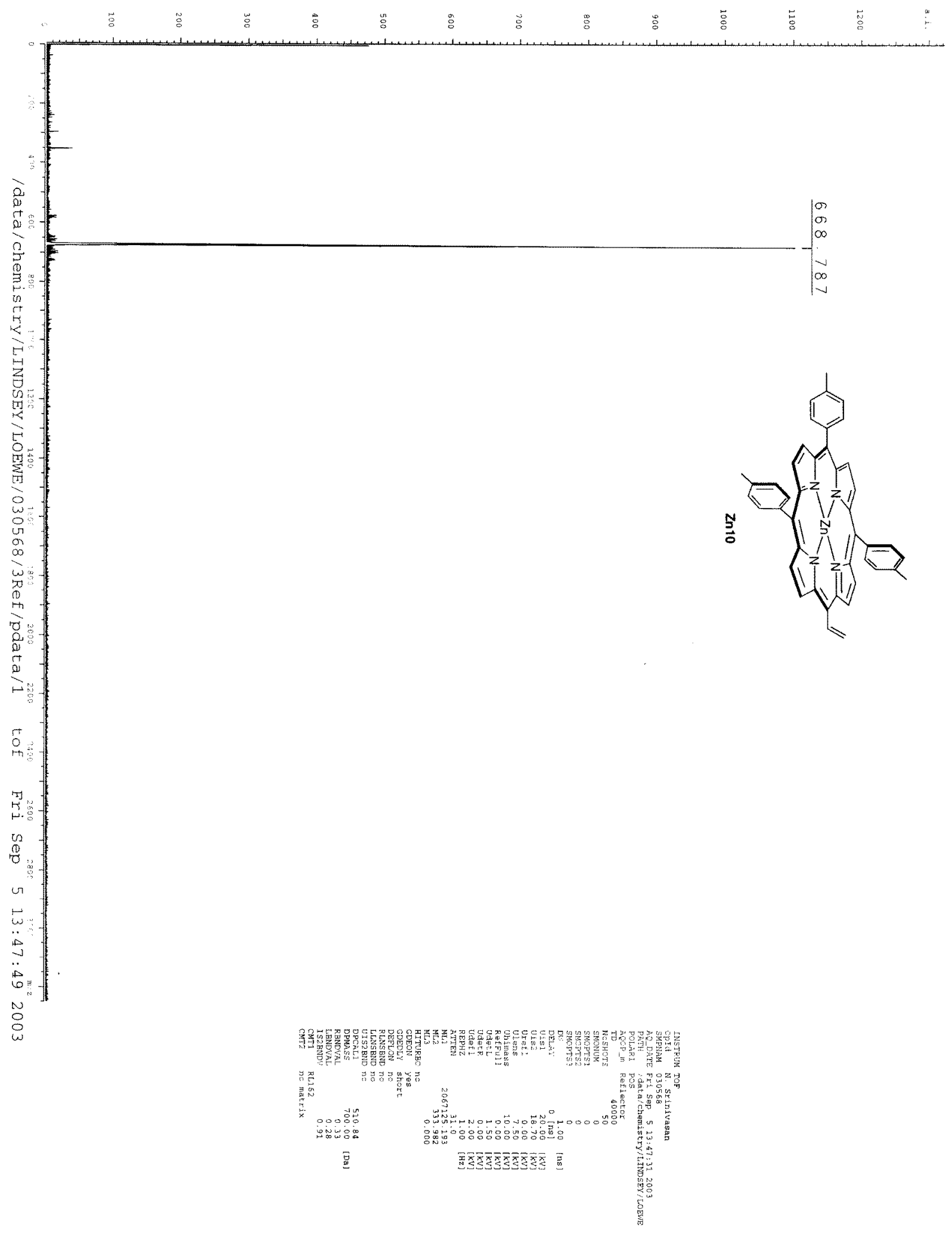




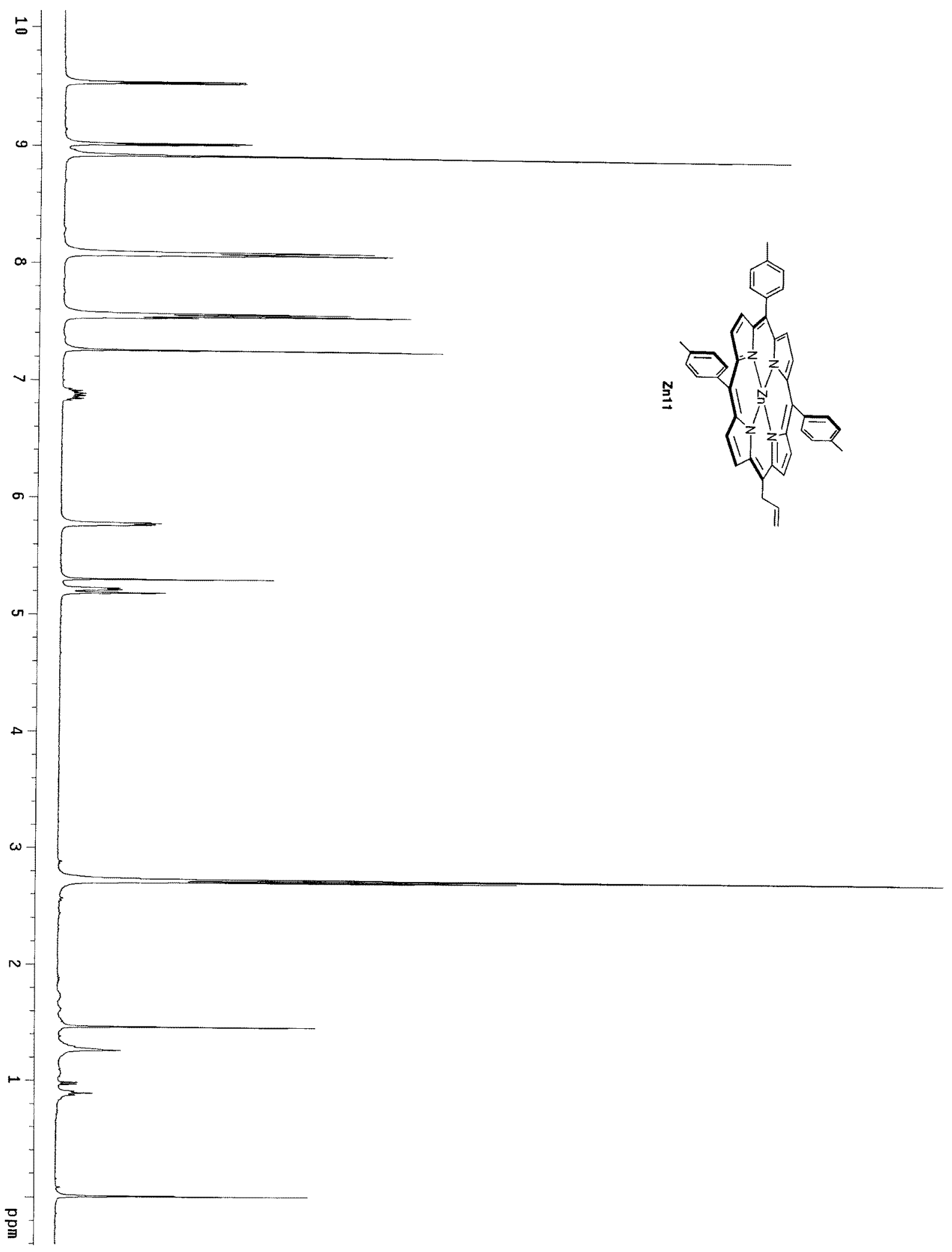




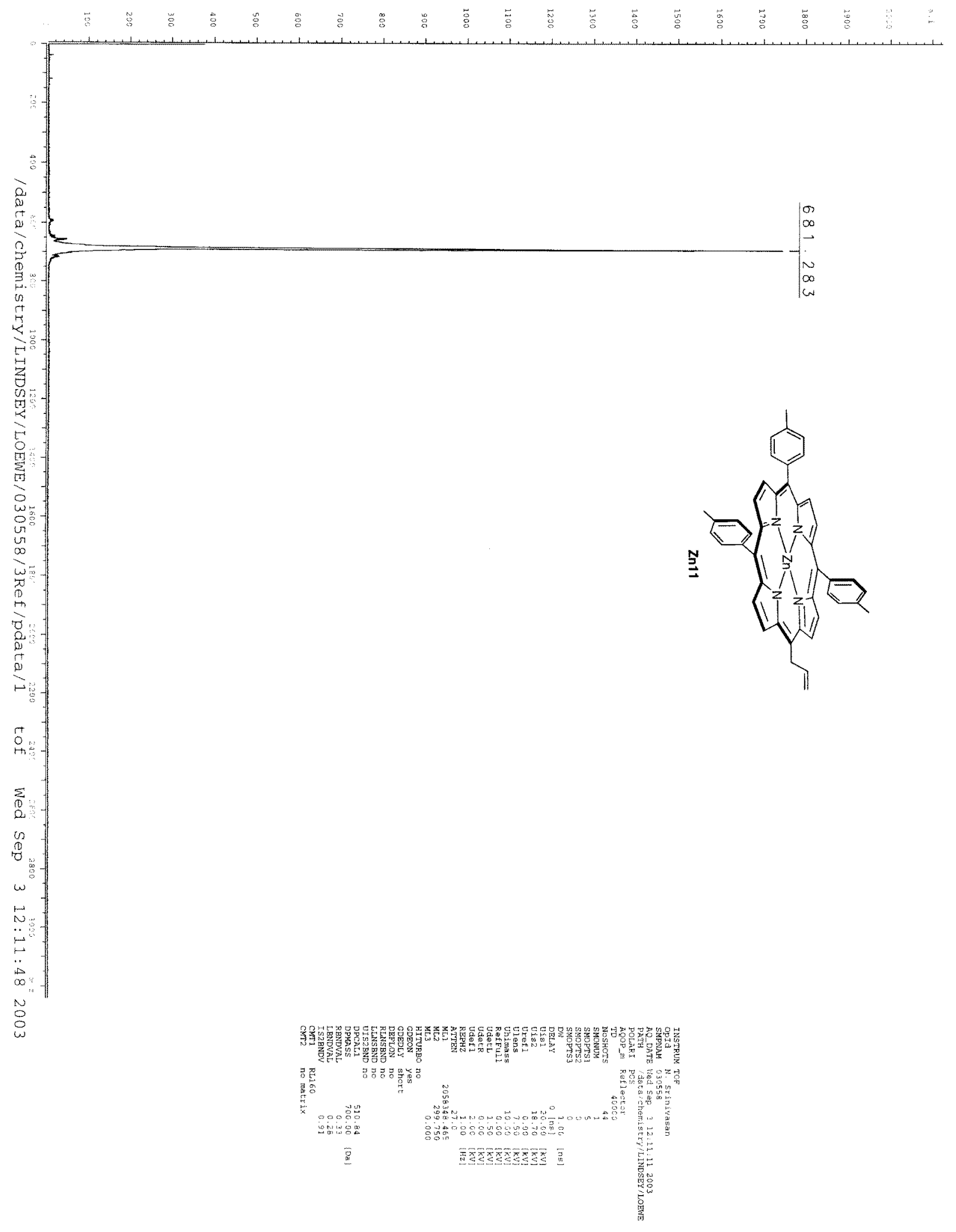

S-23 


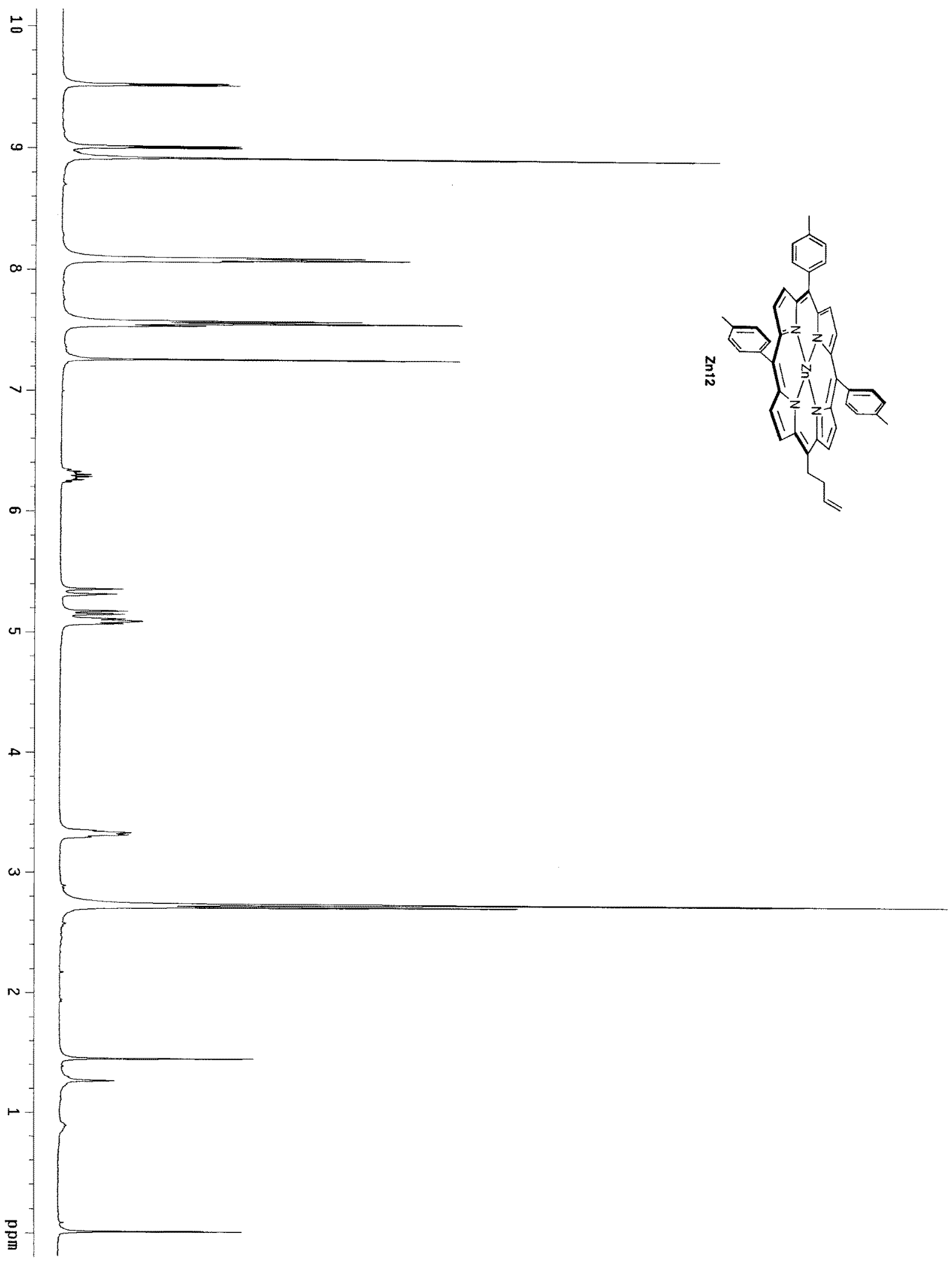




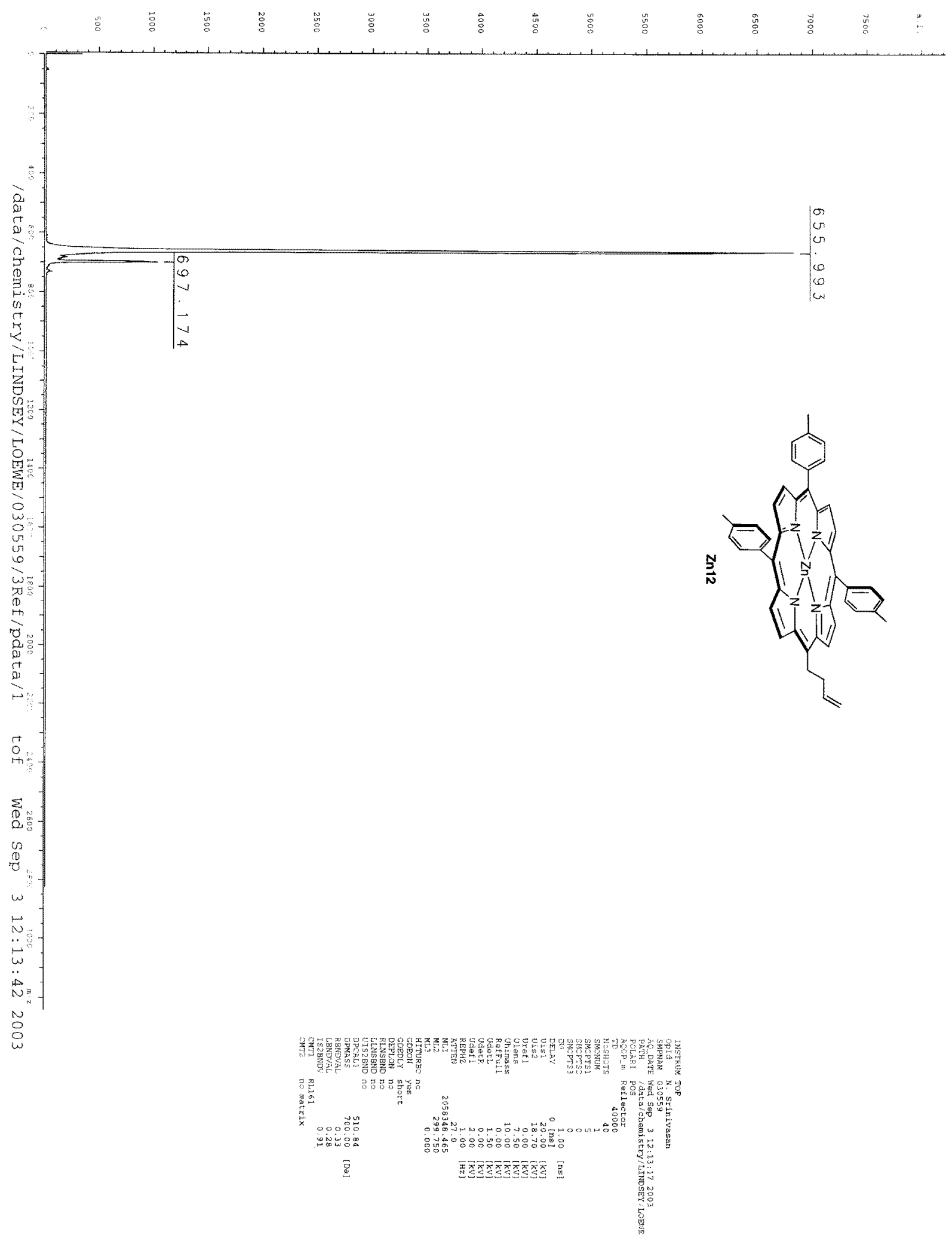

S-25 


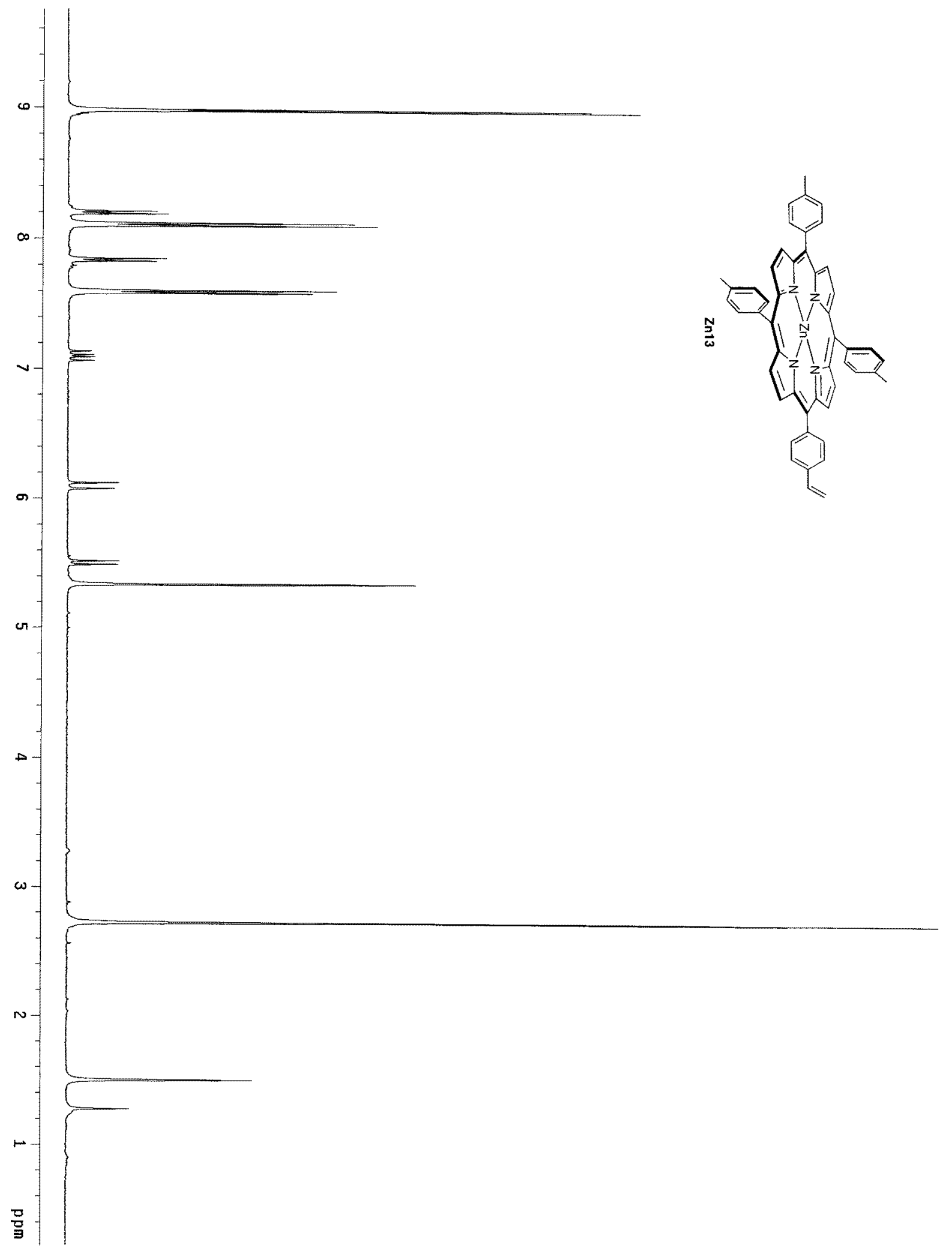




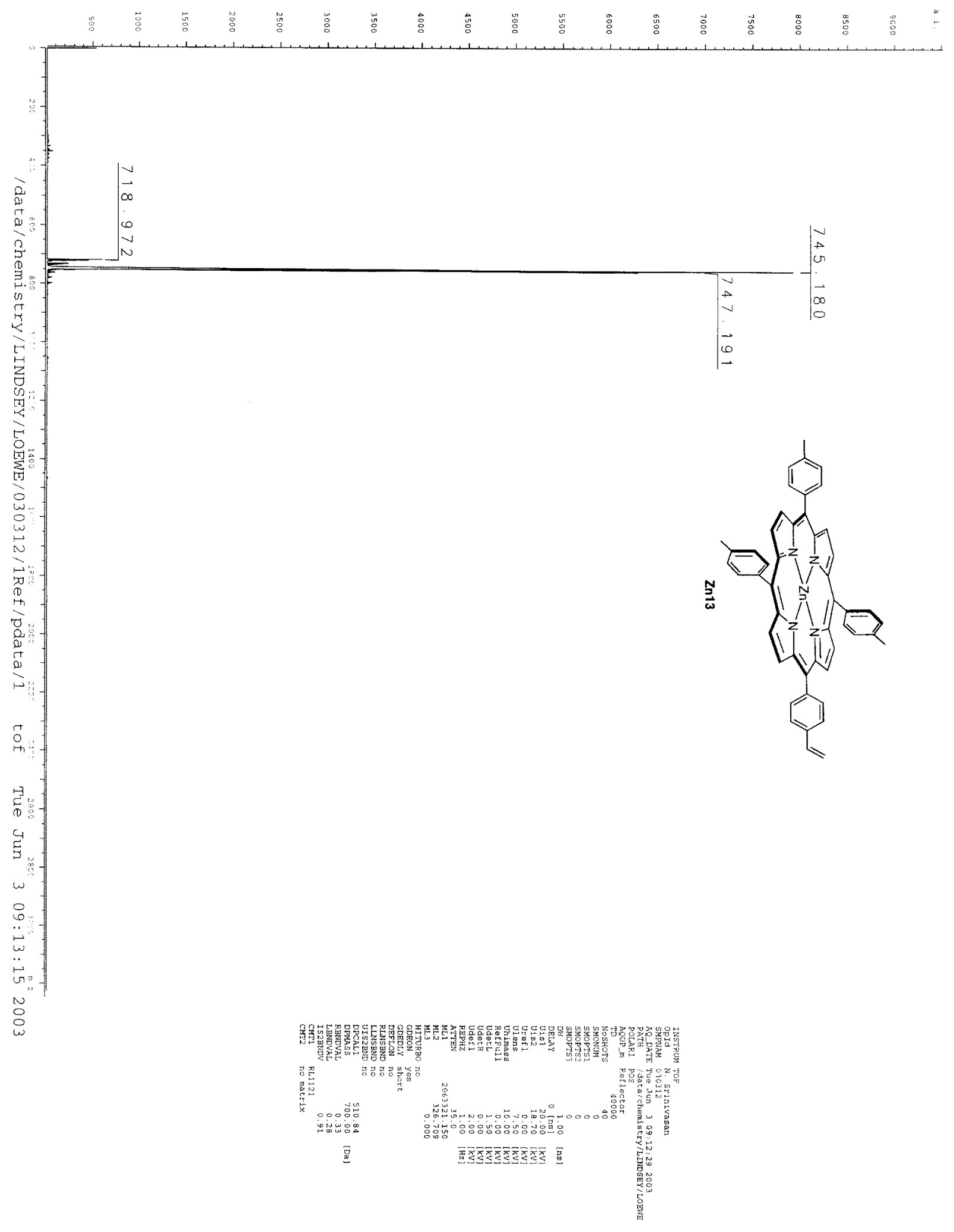




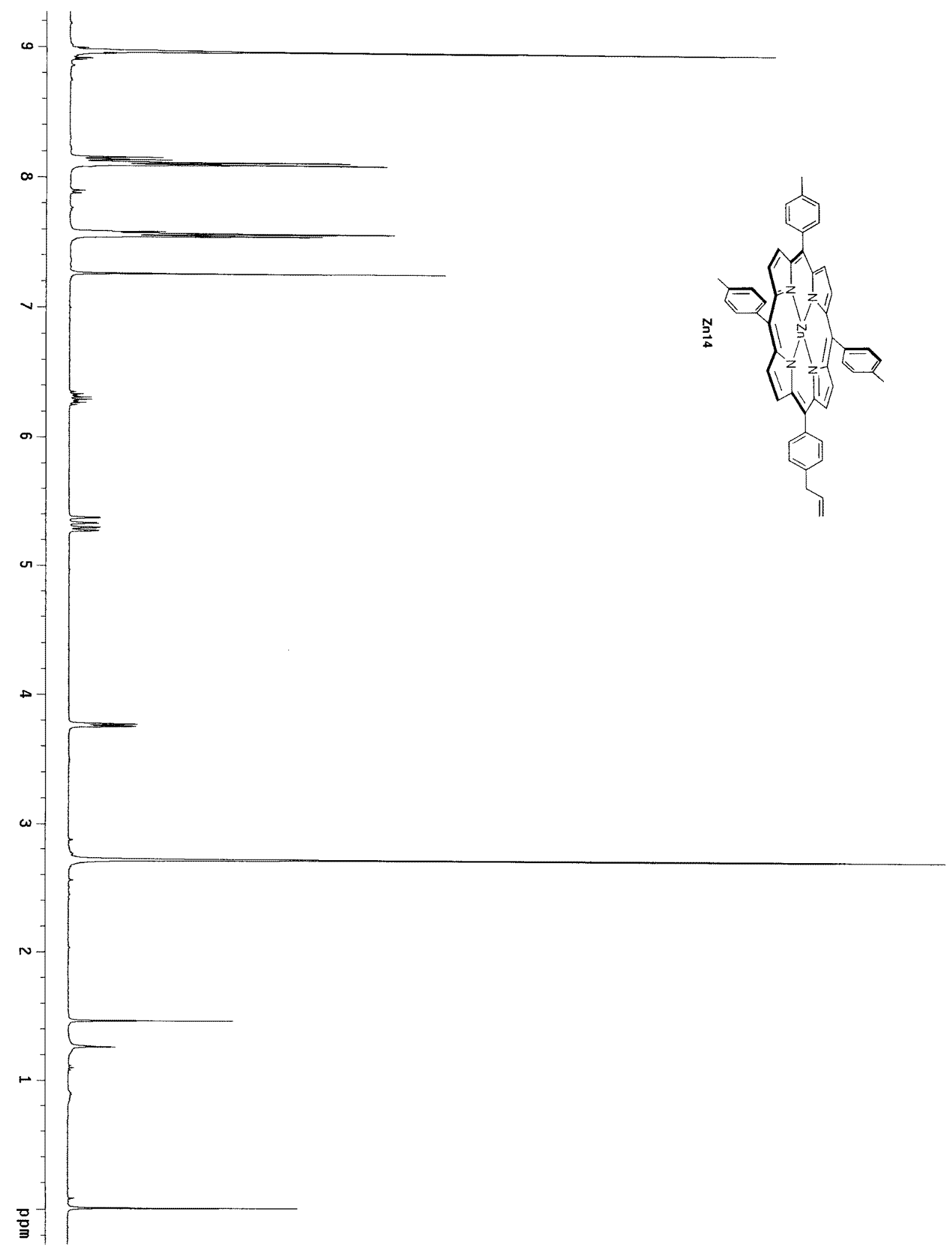




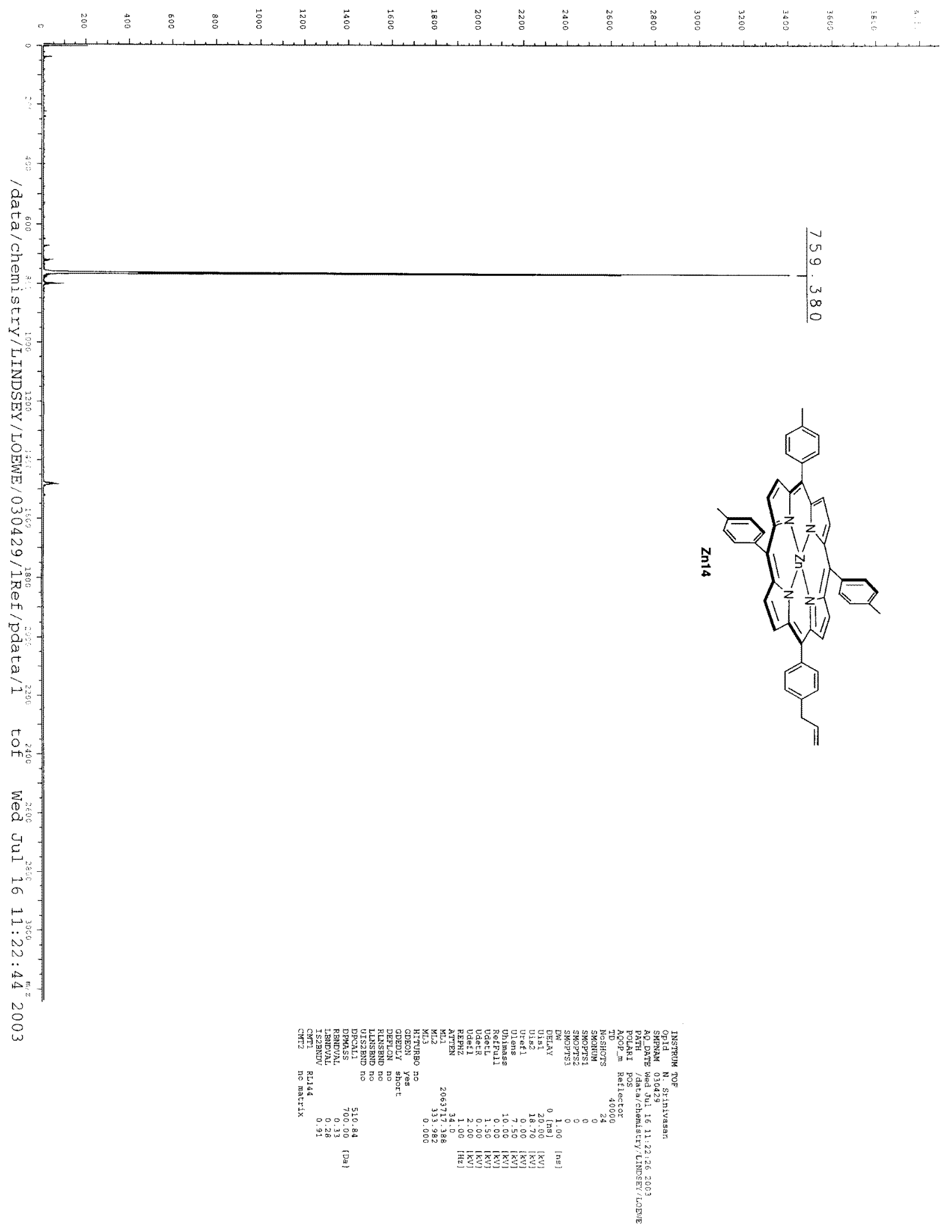




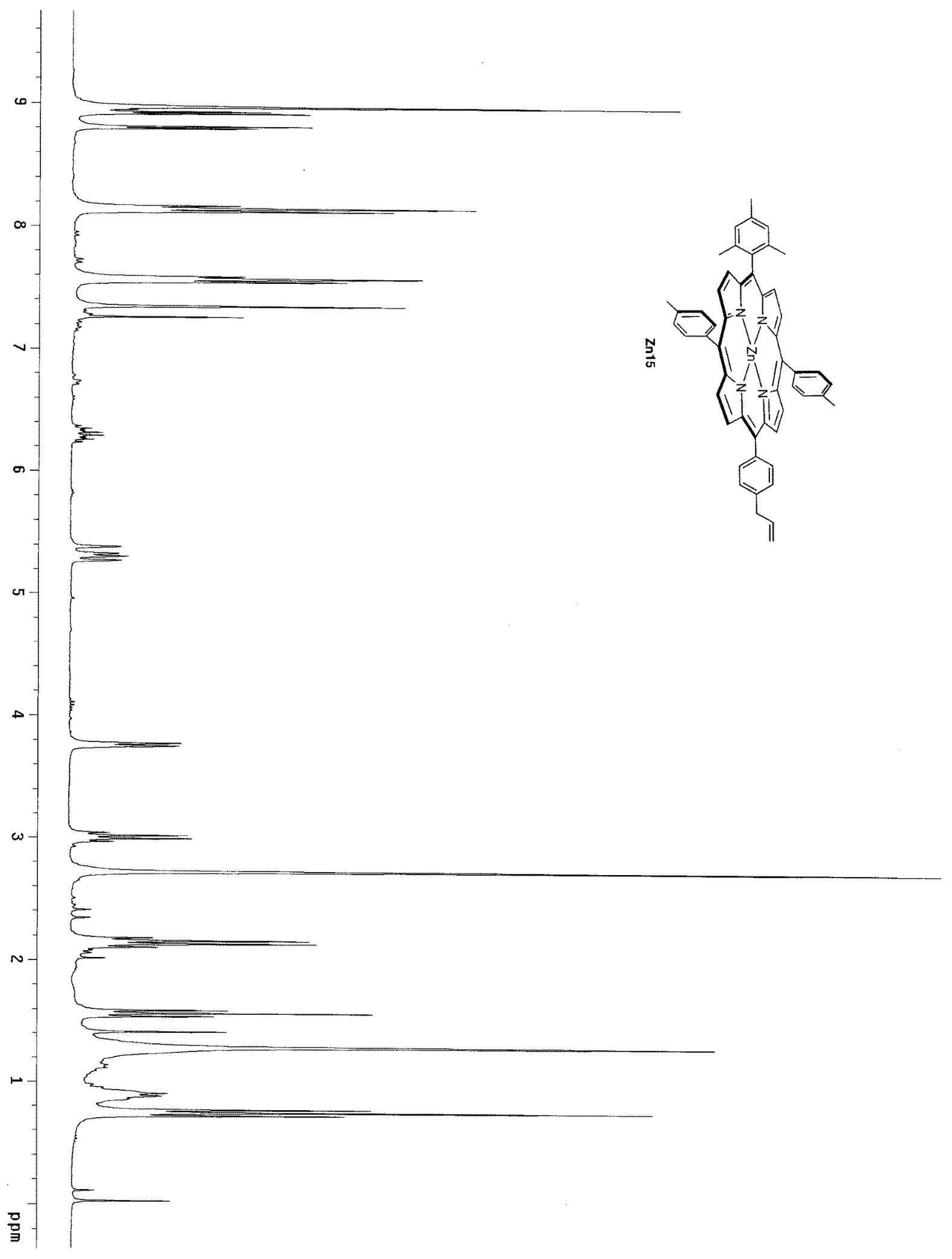




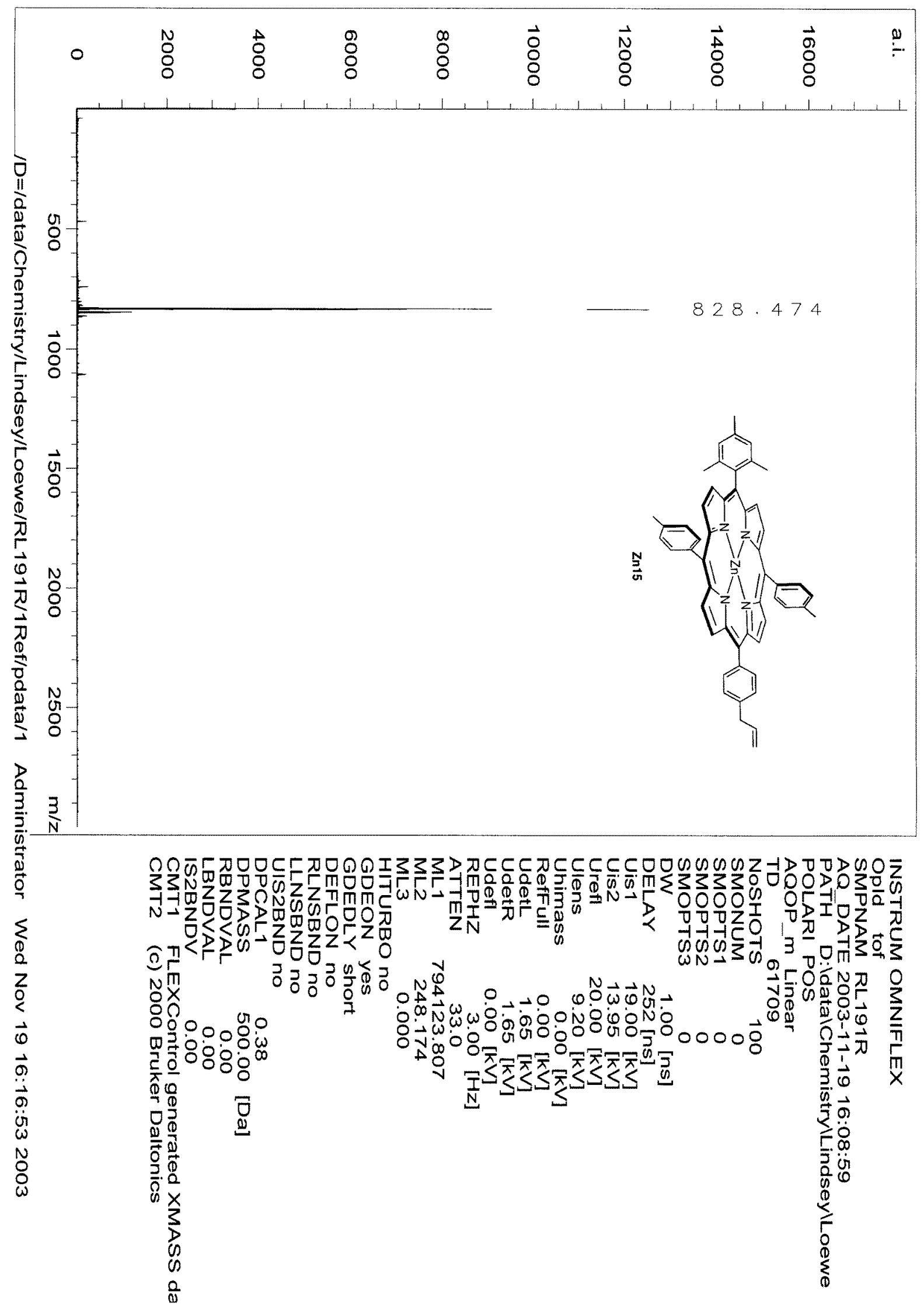




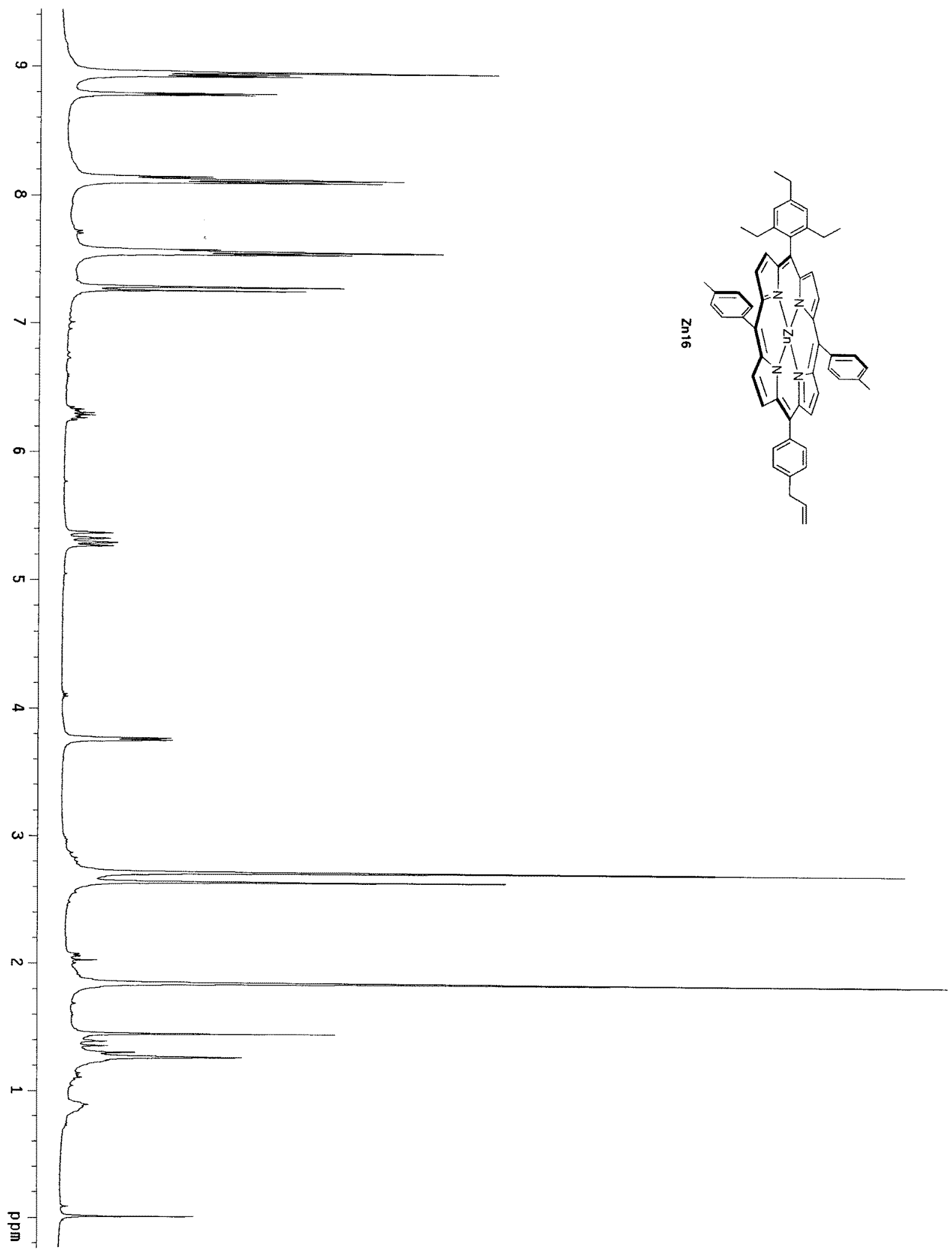




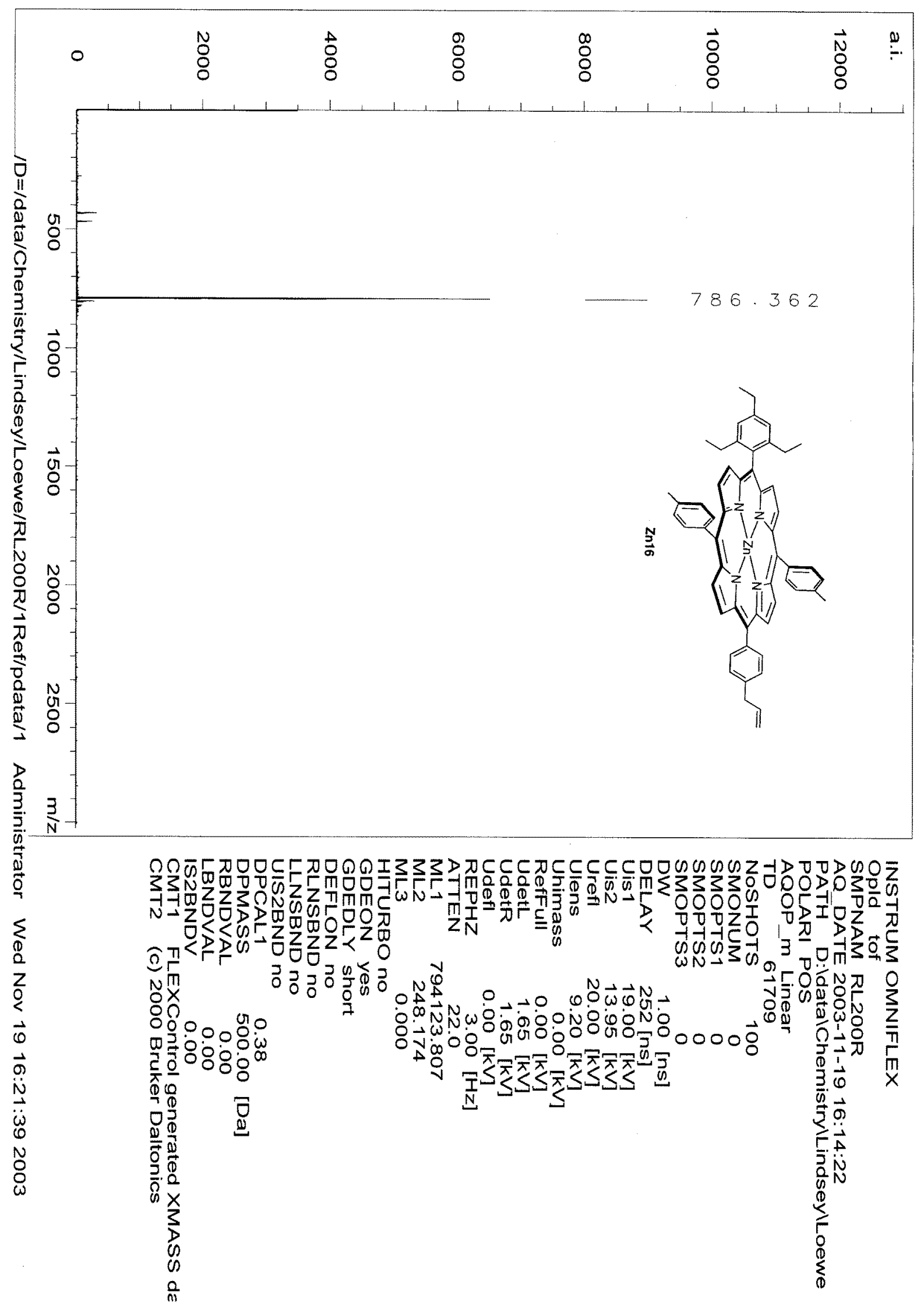




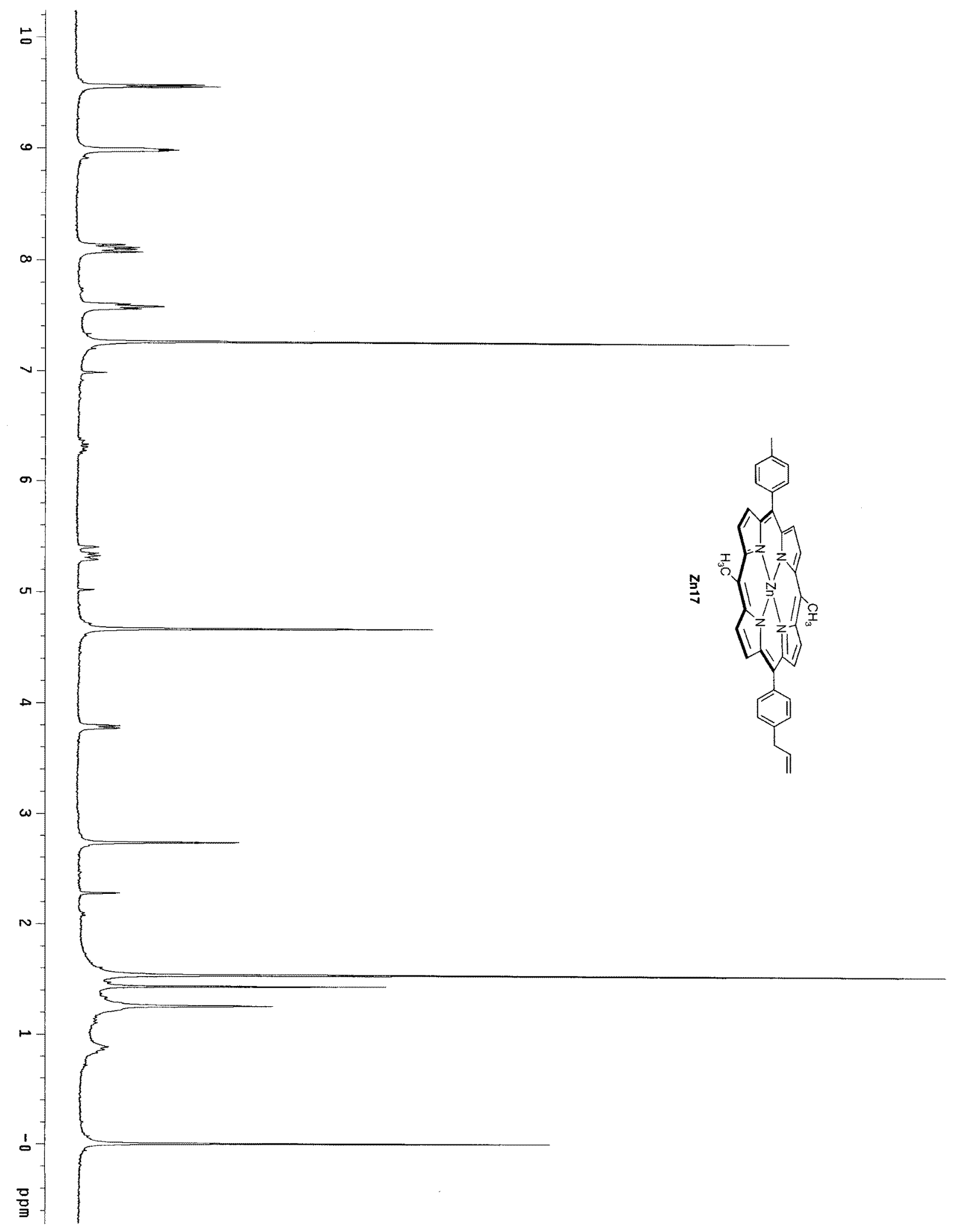




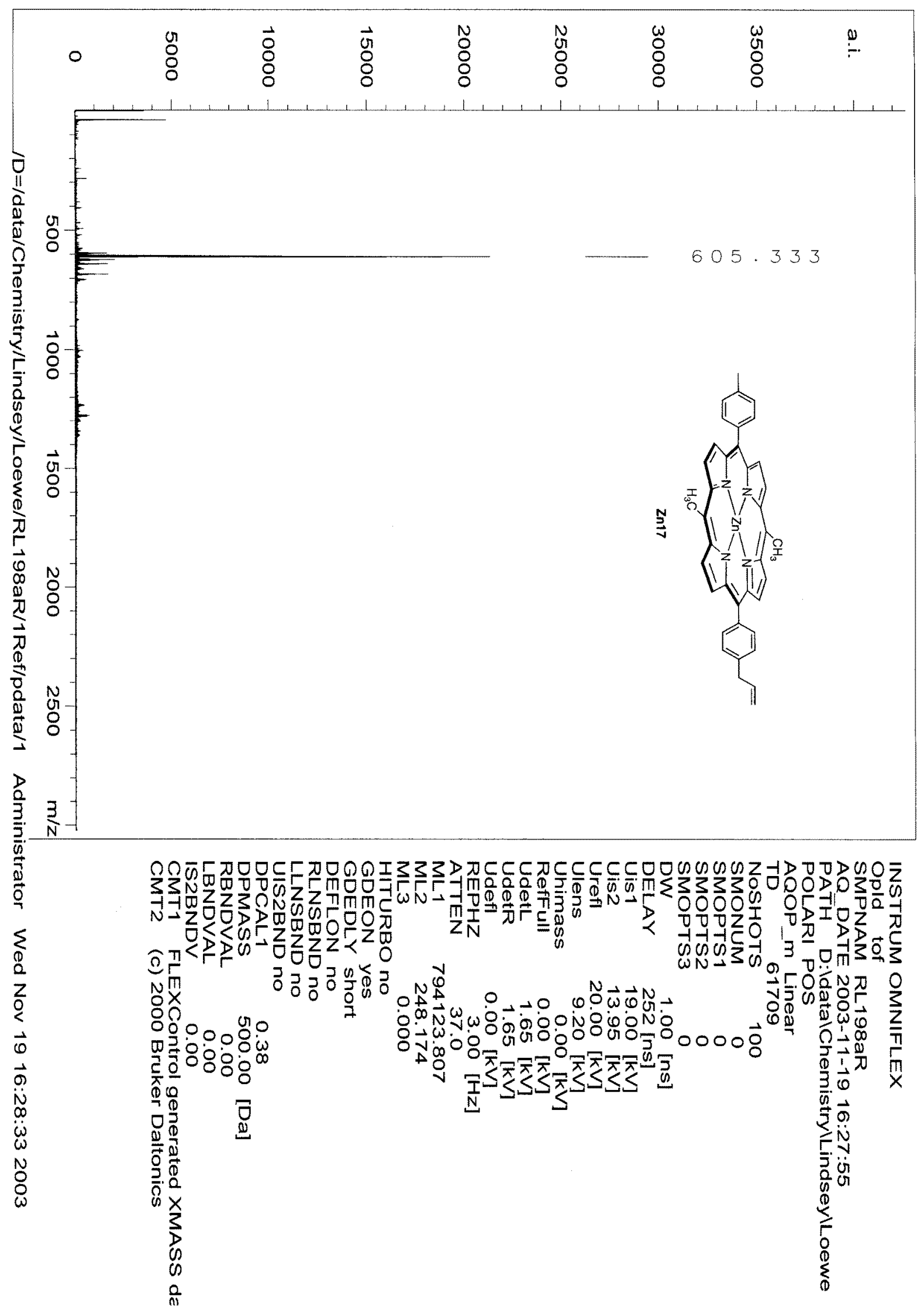




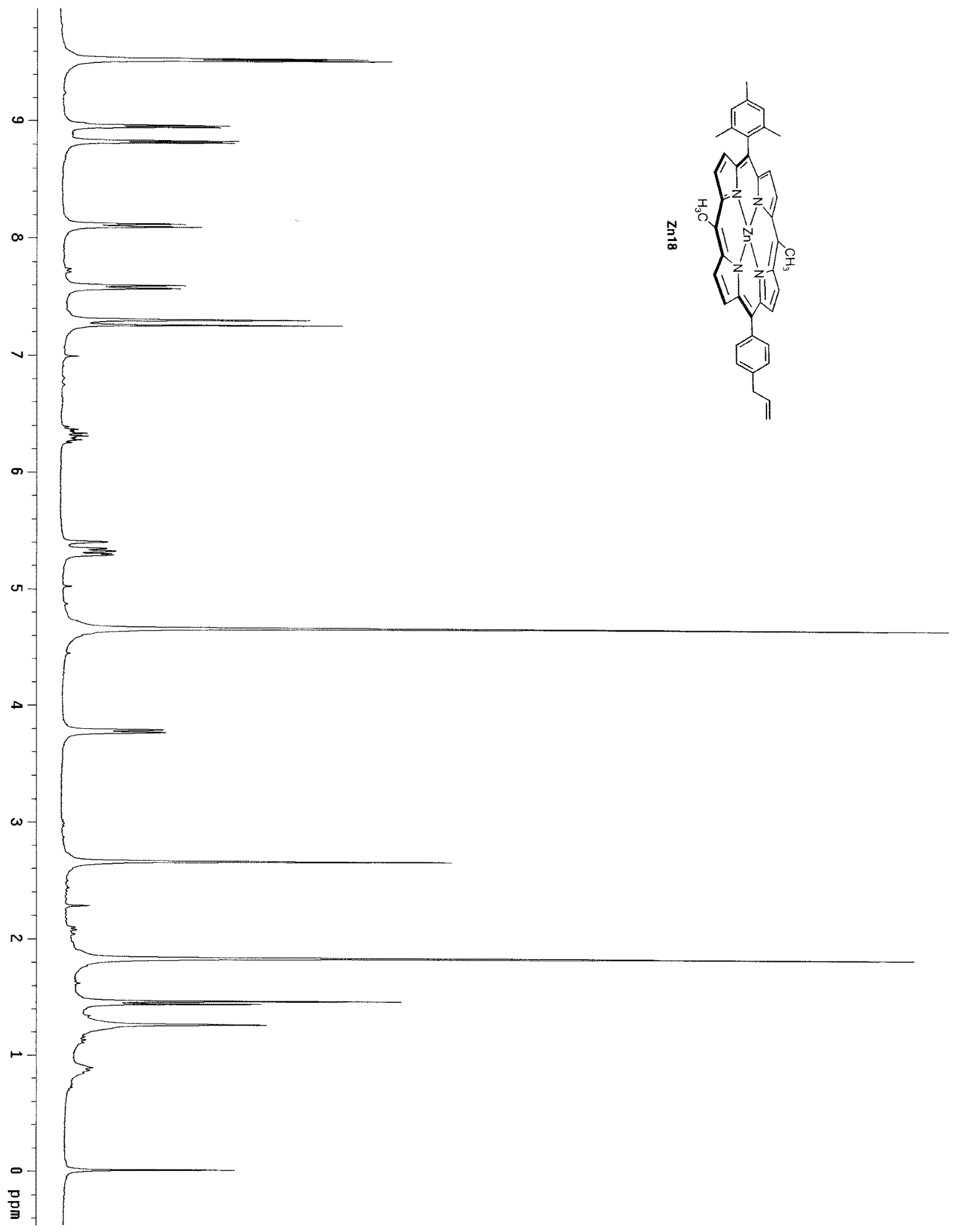




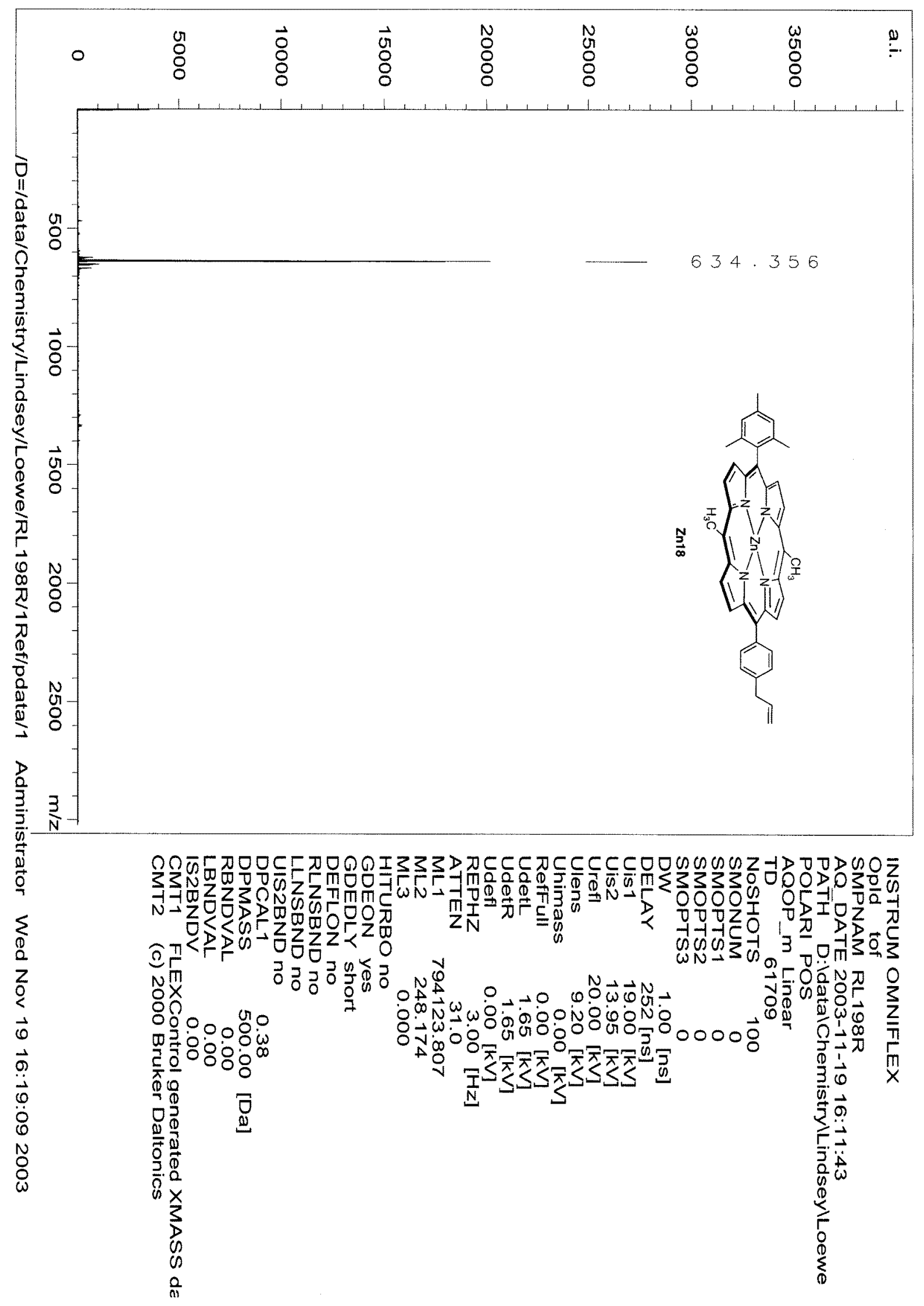




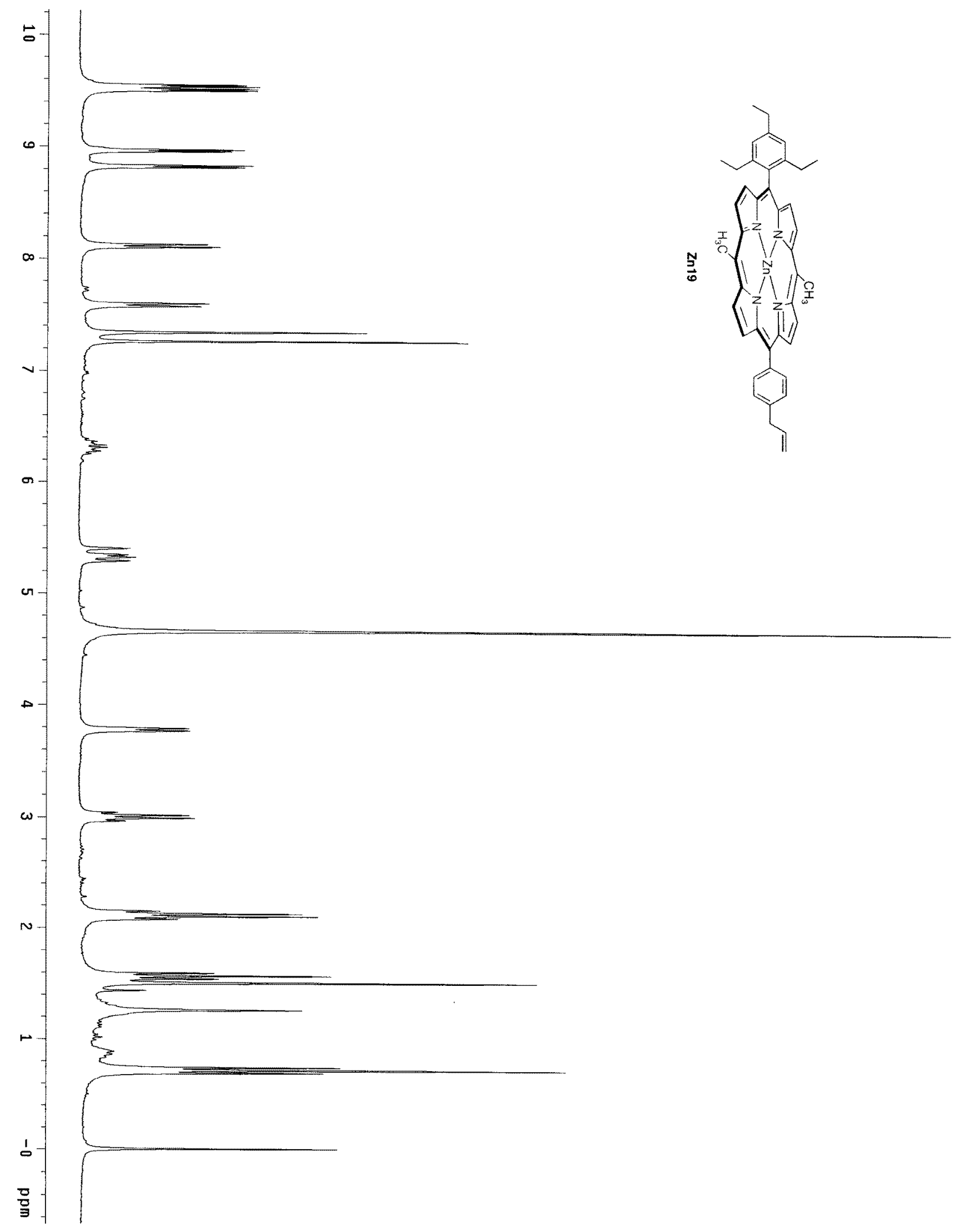




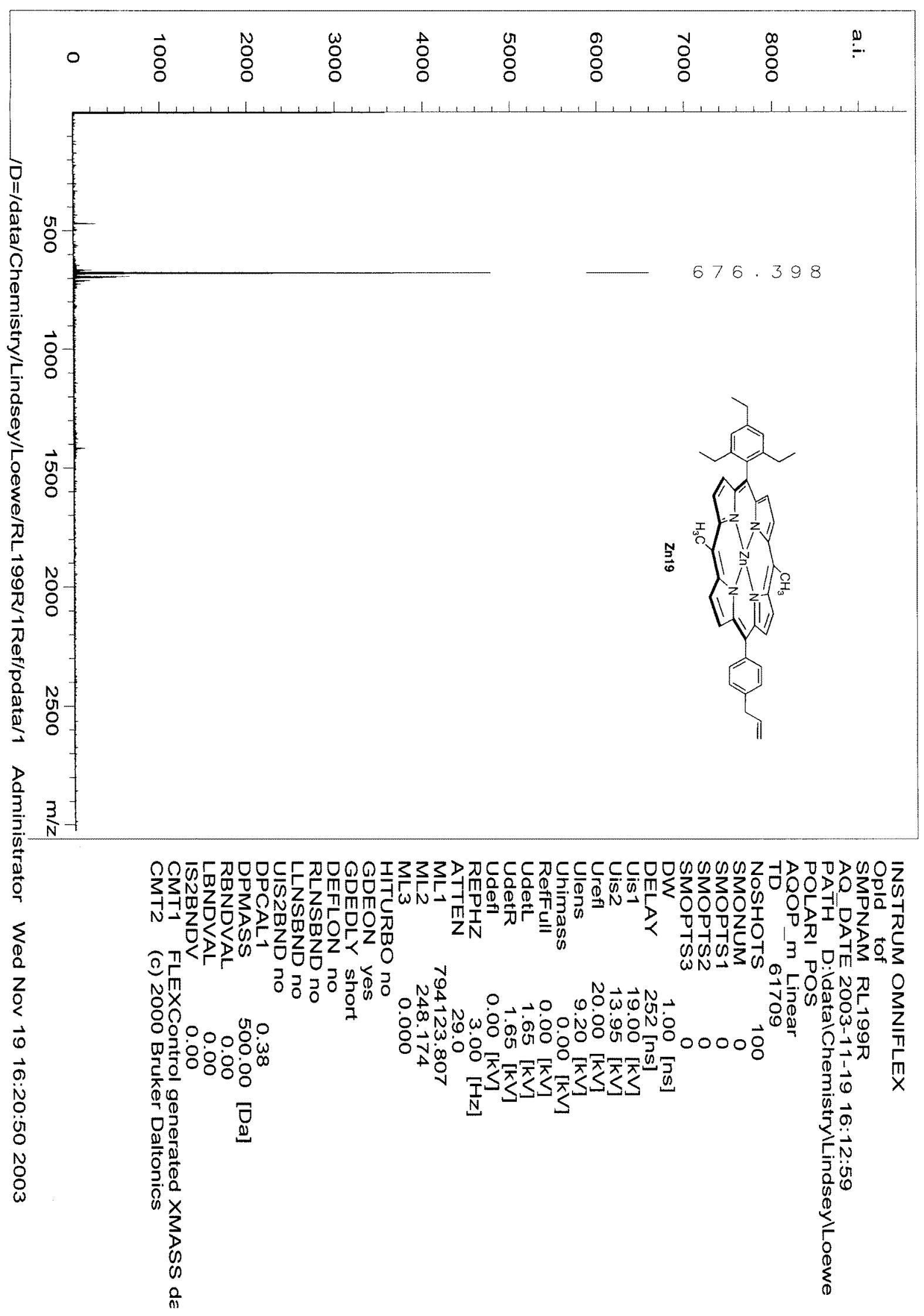




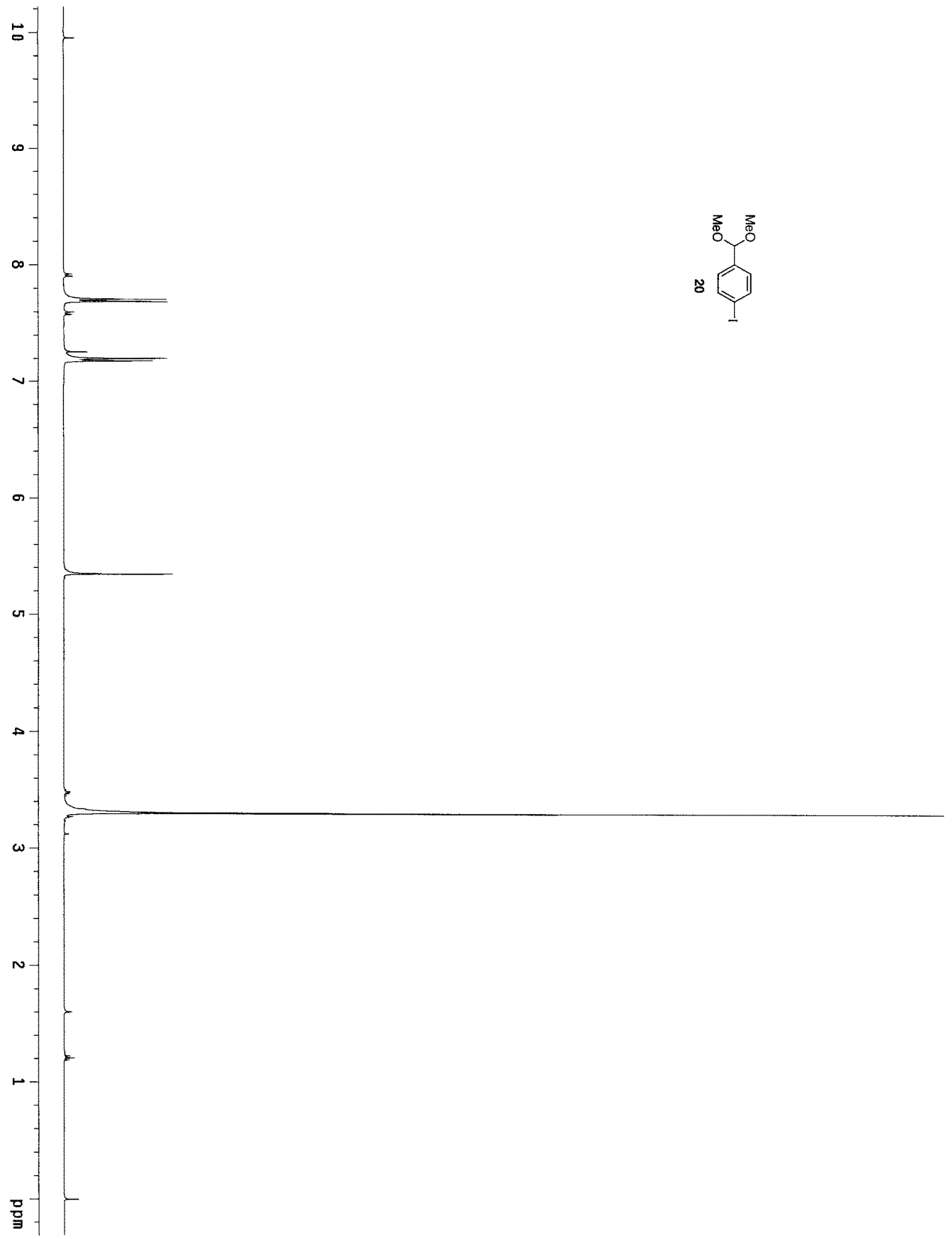




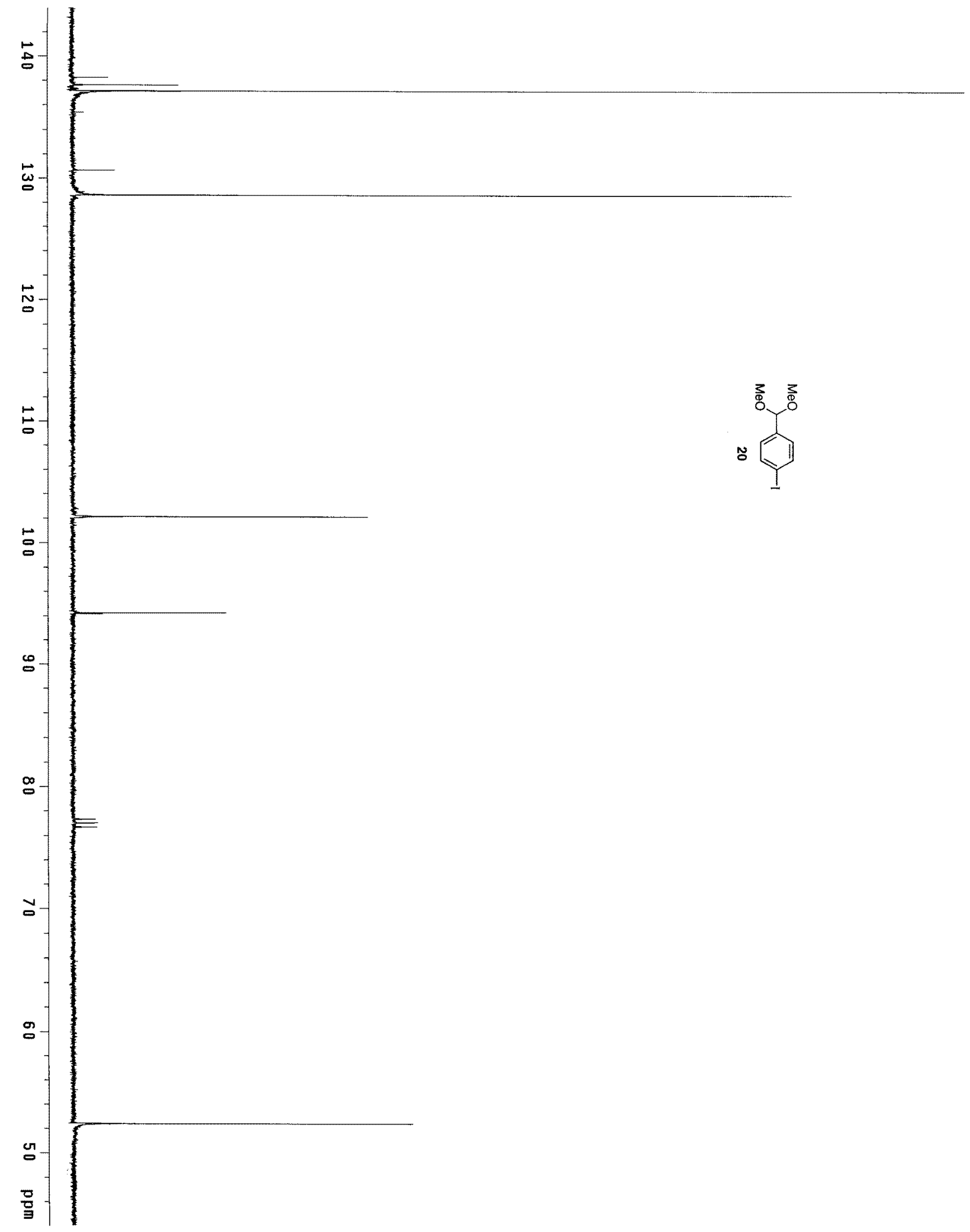




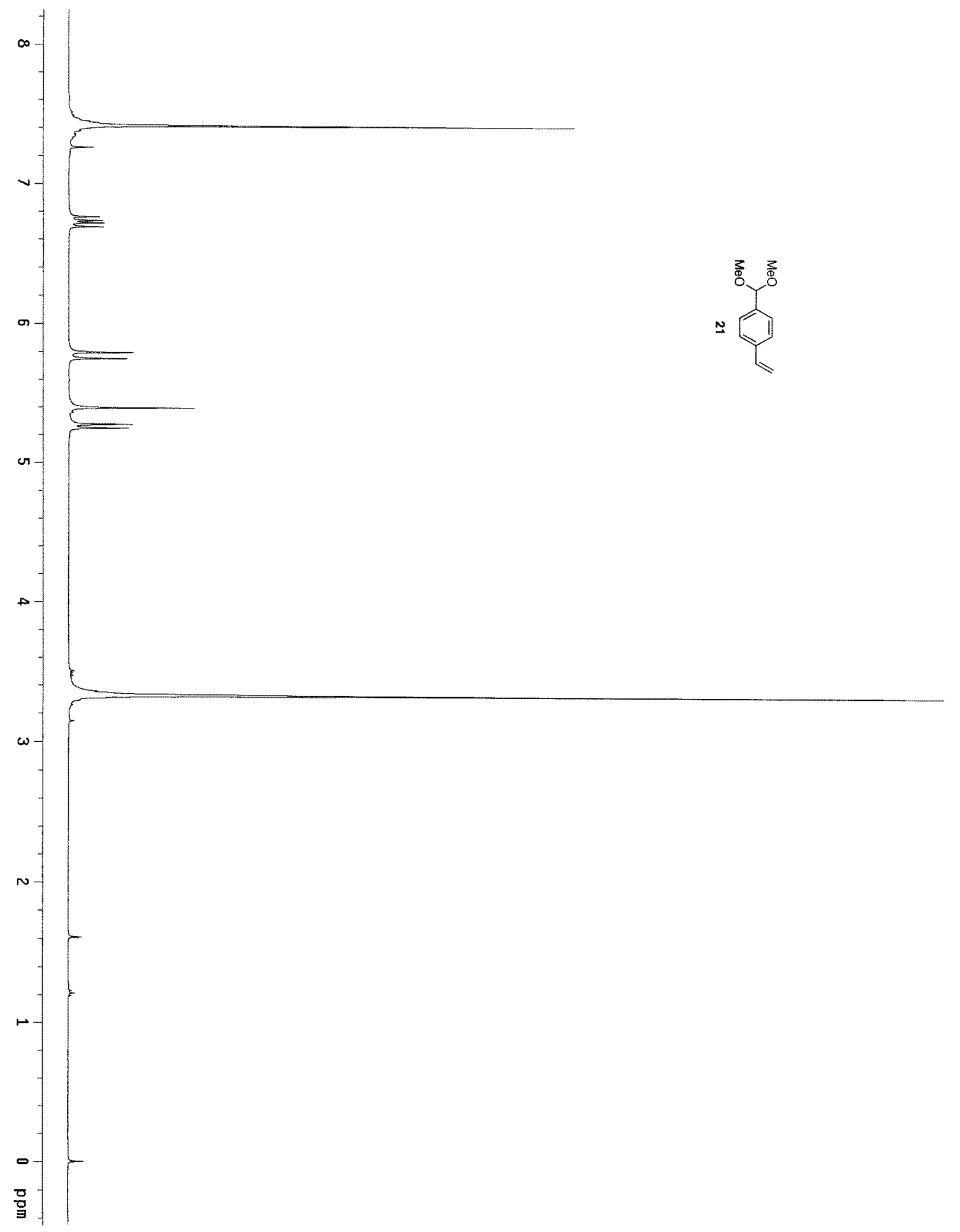




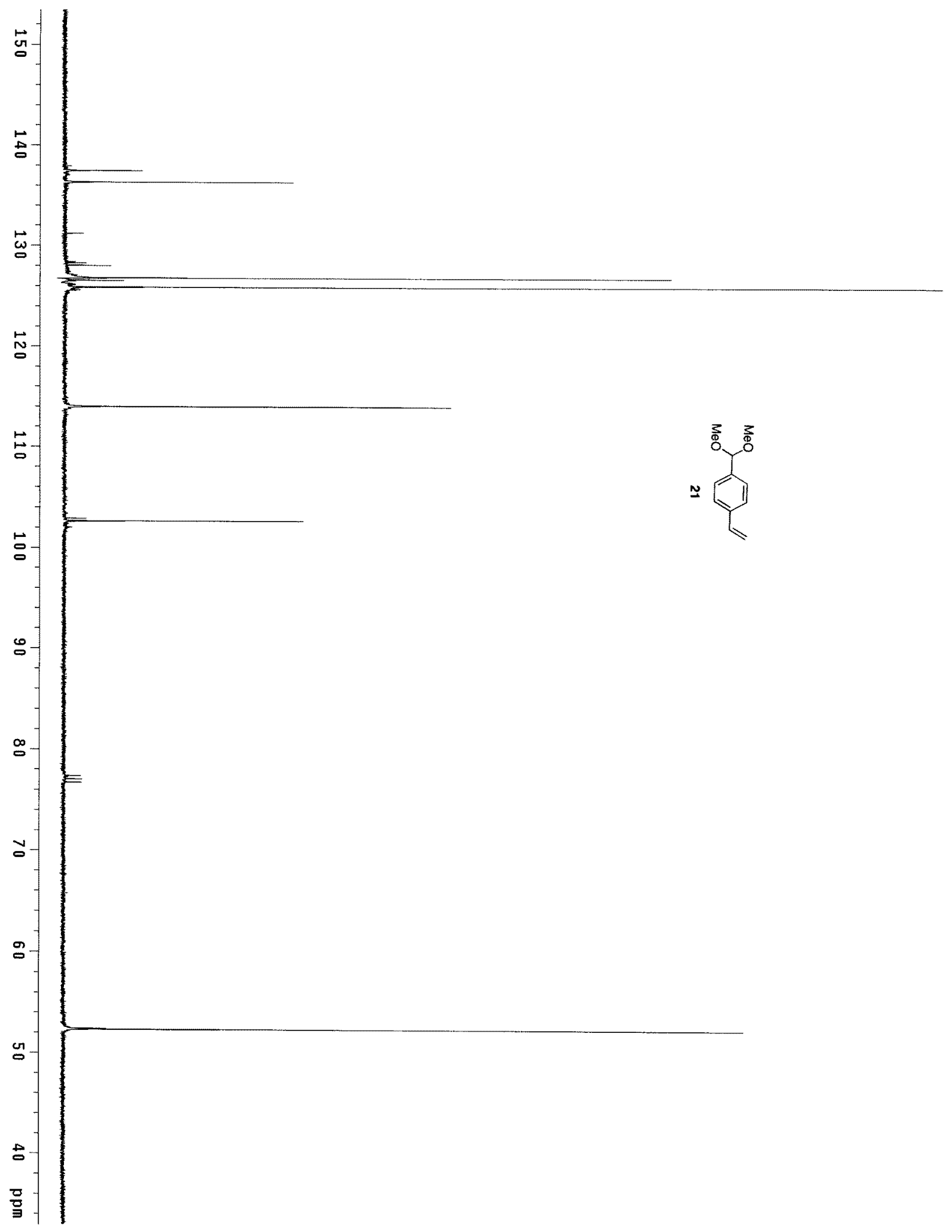




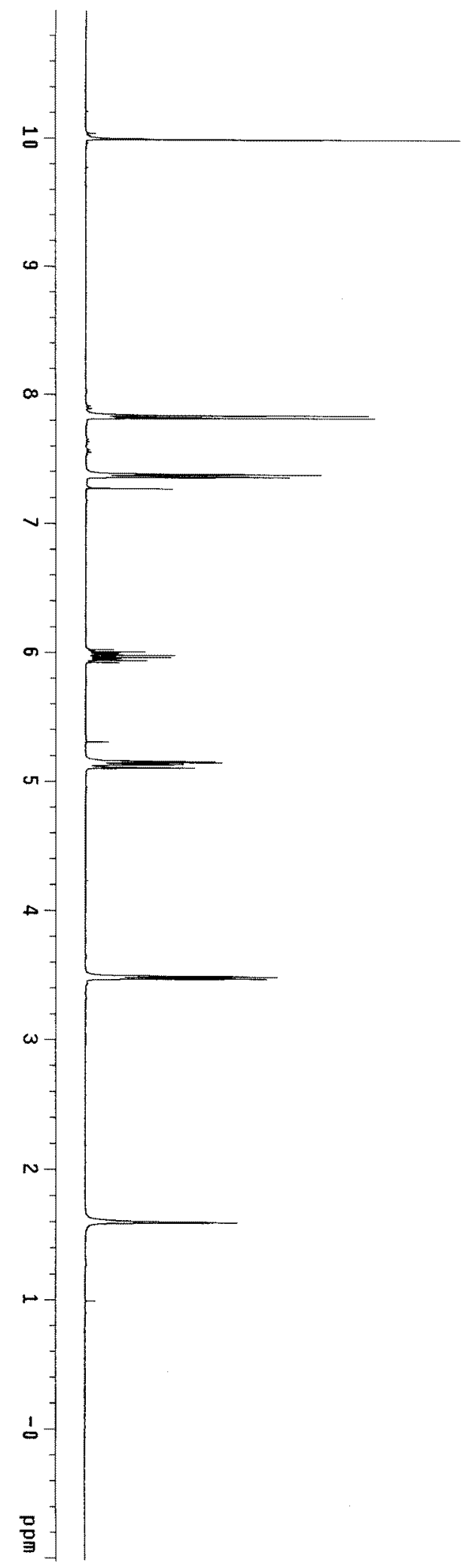




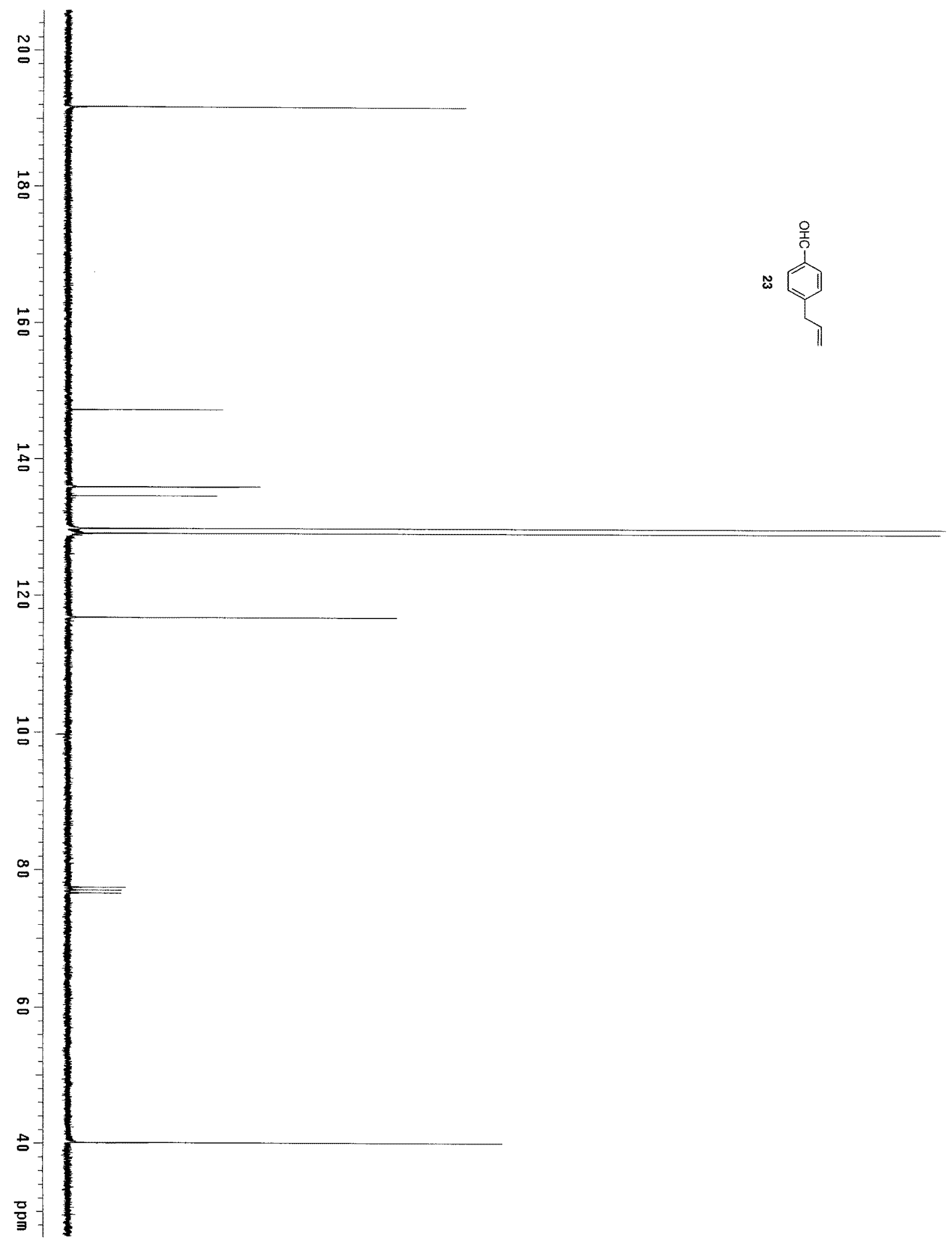




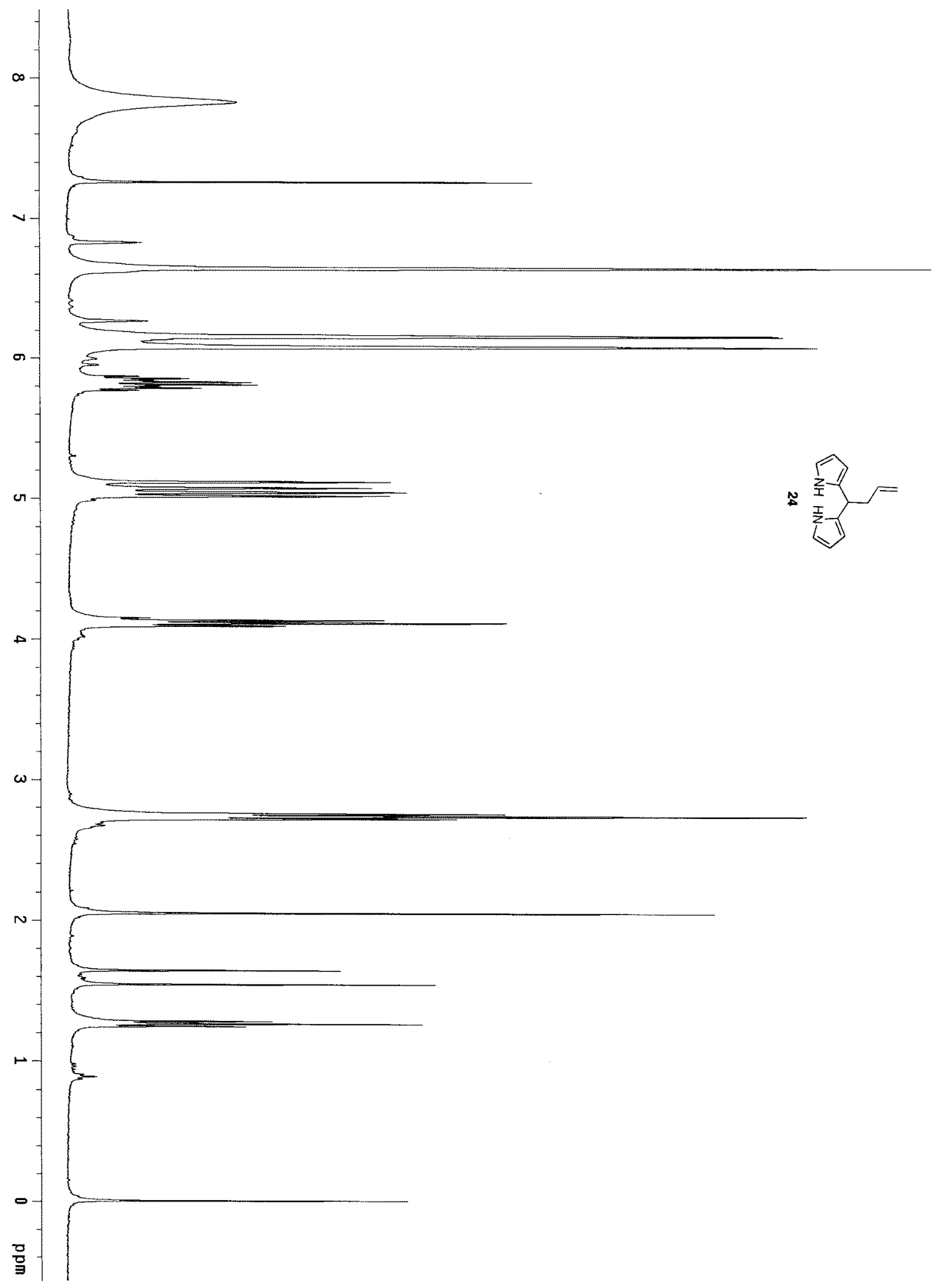




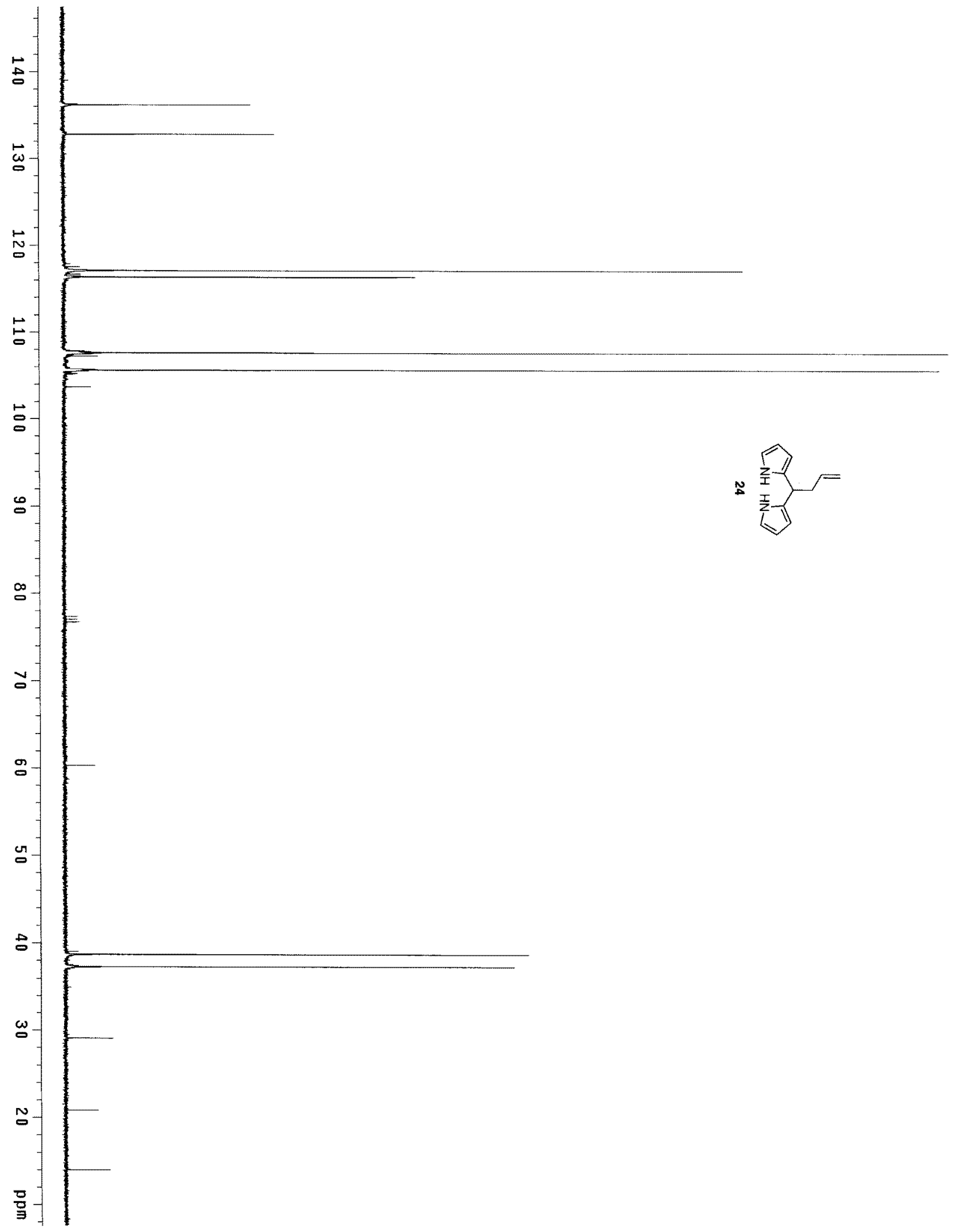




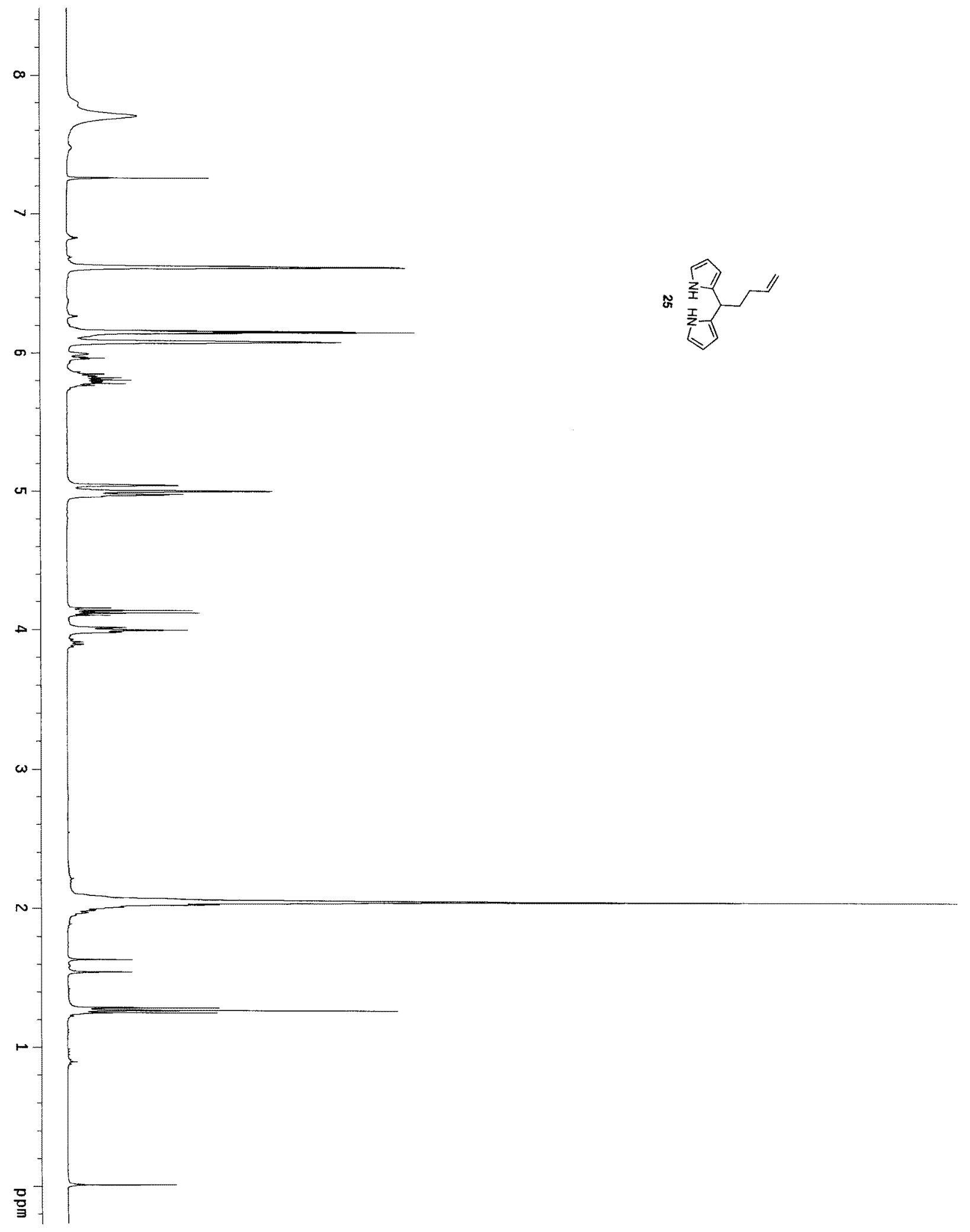




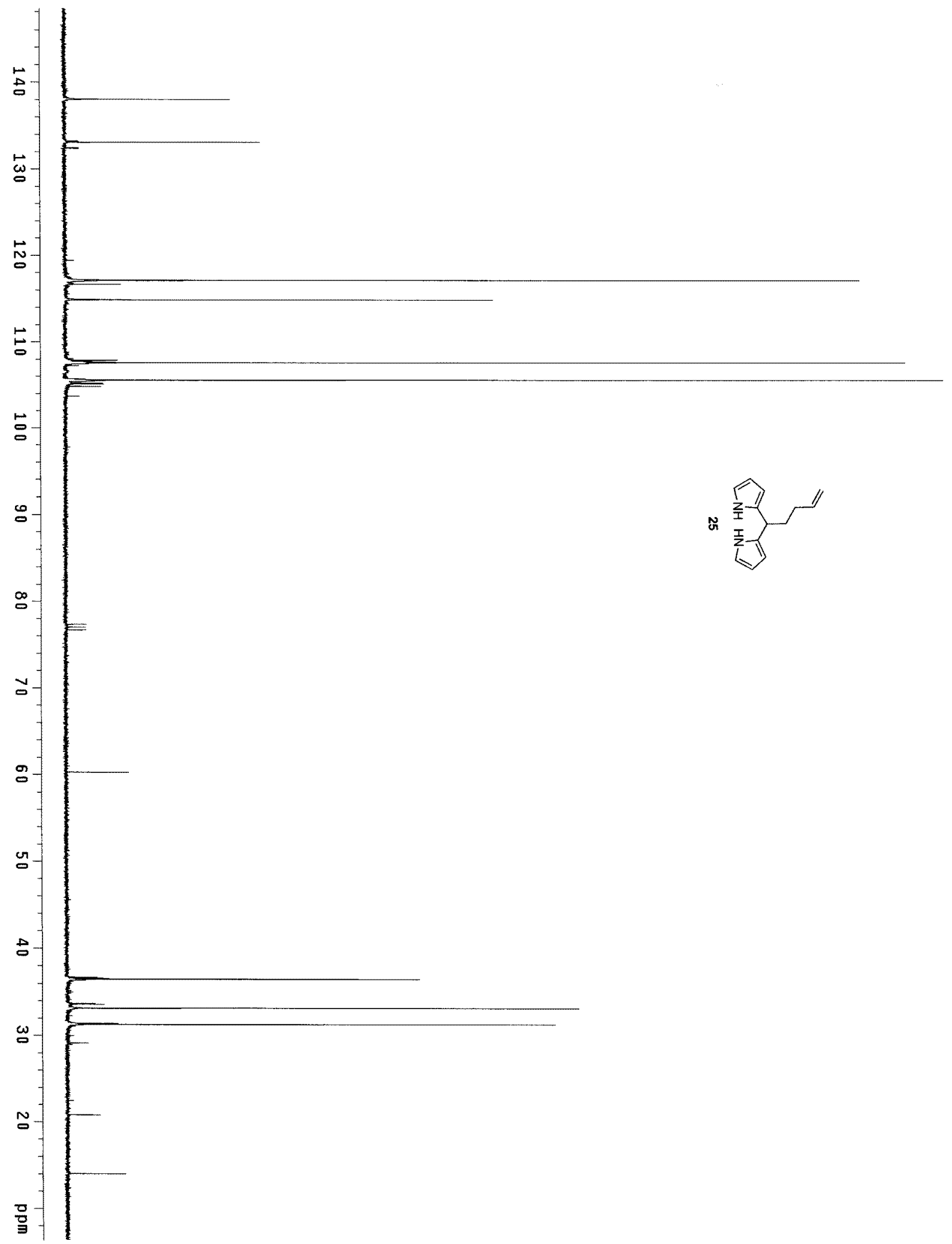




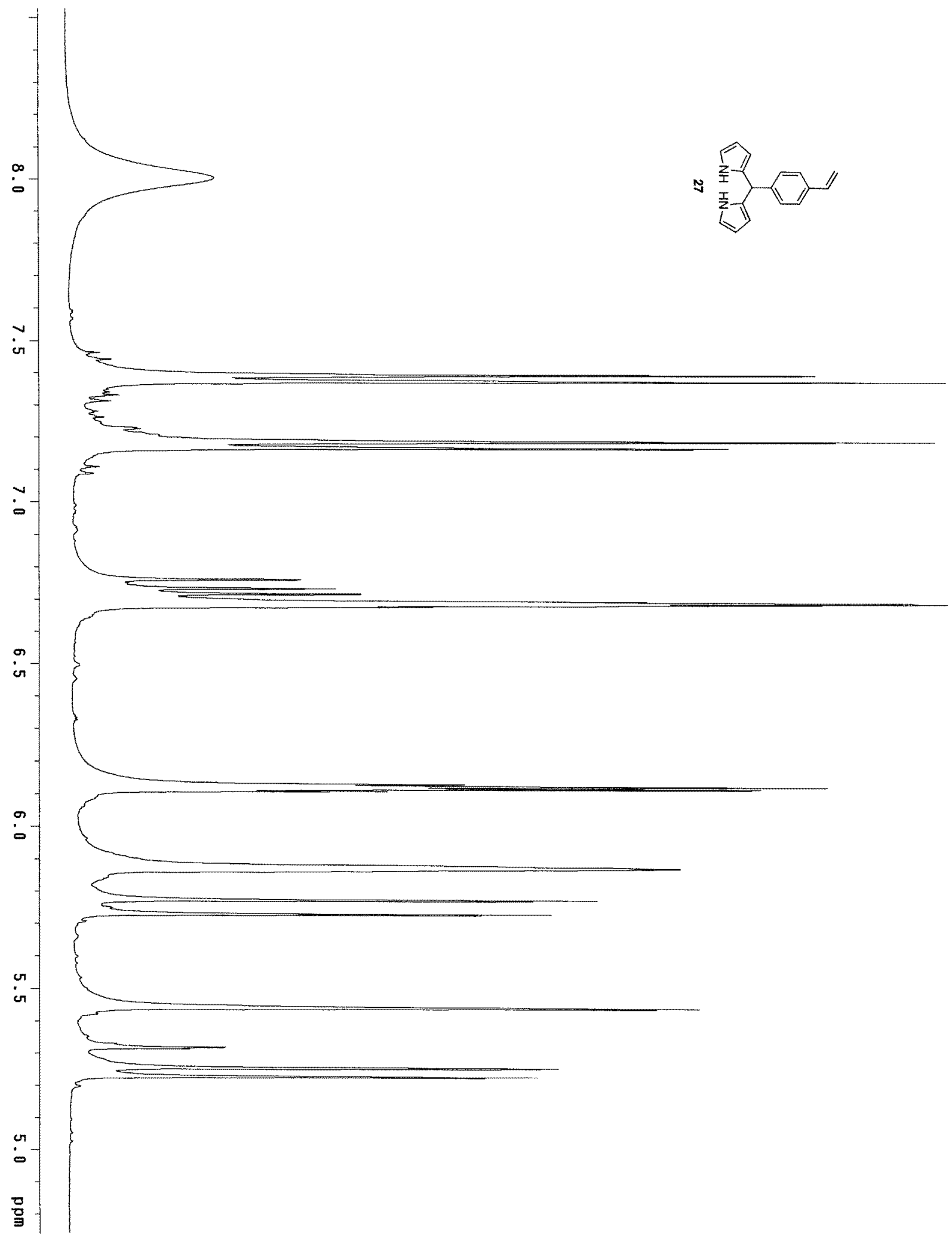




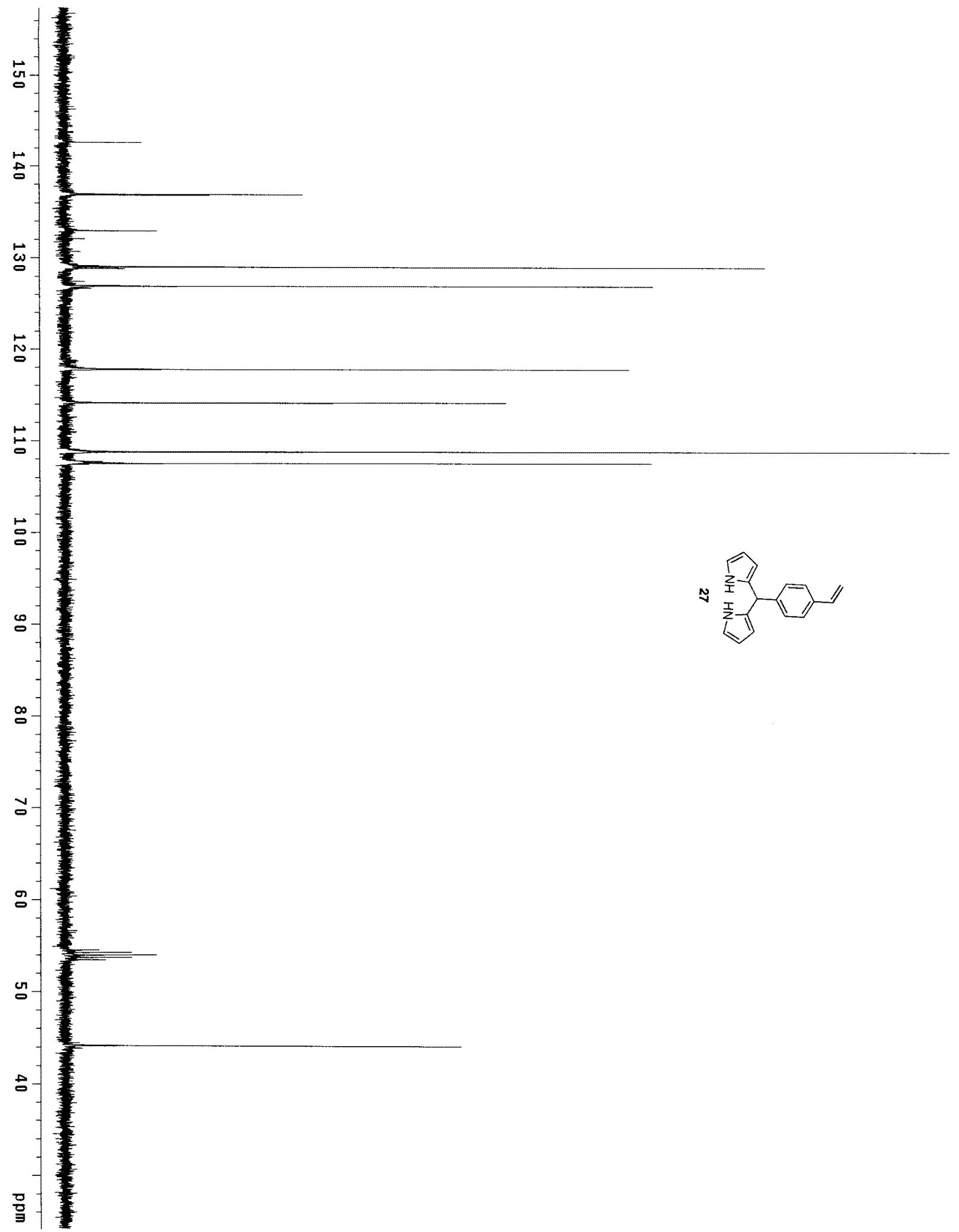




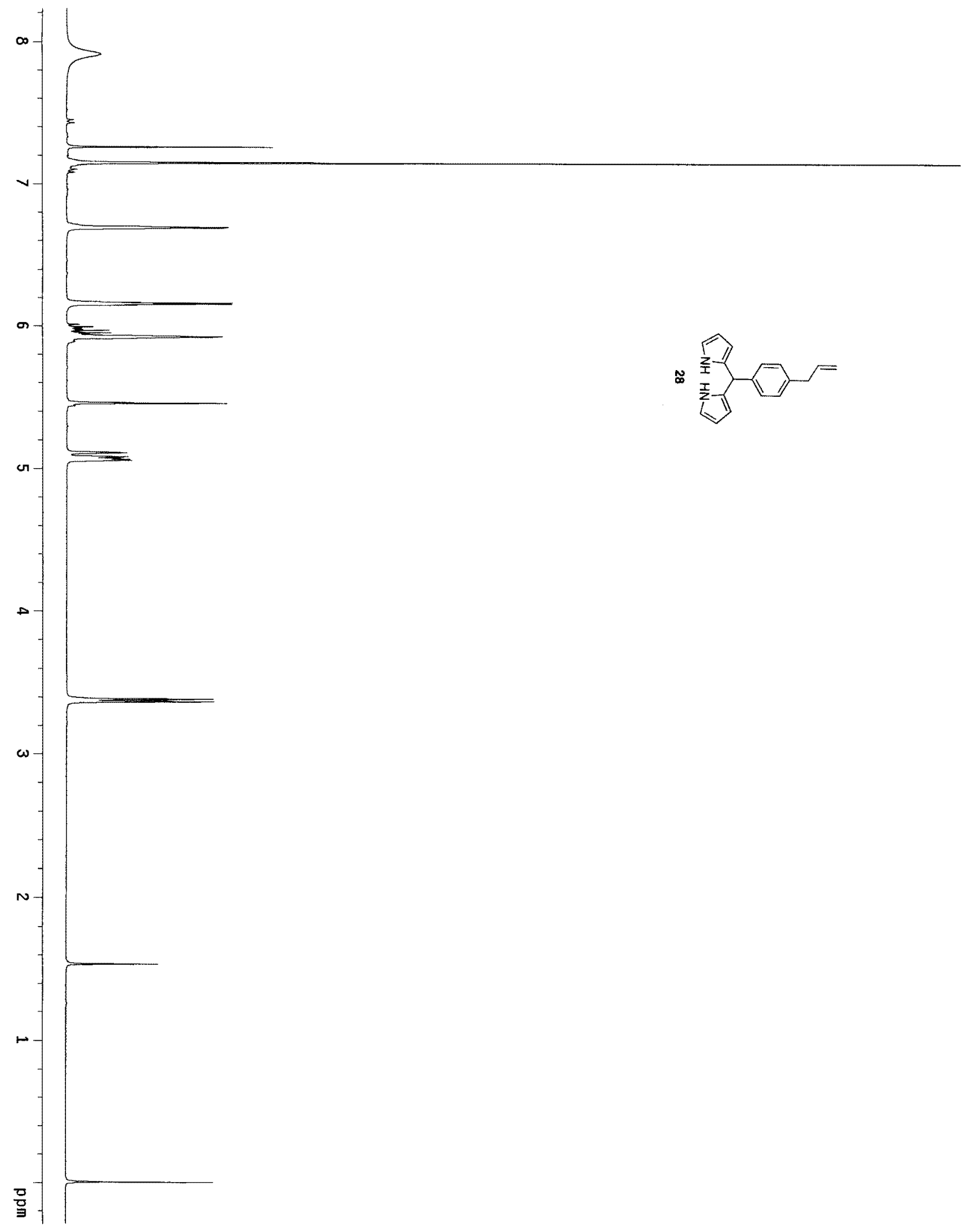




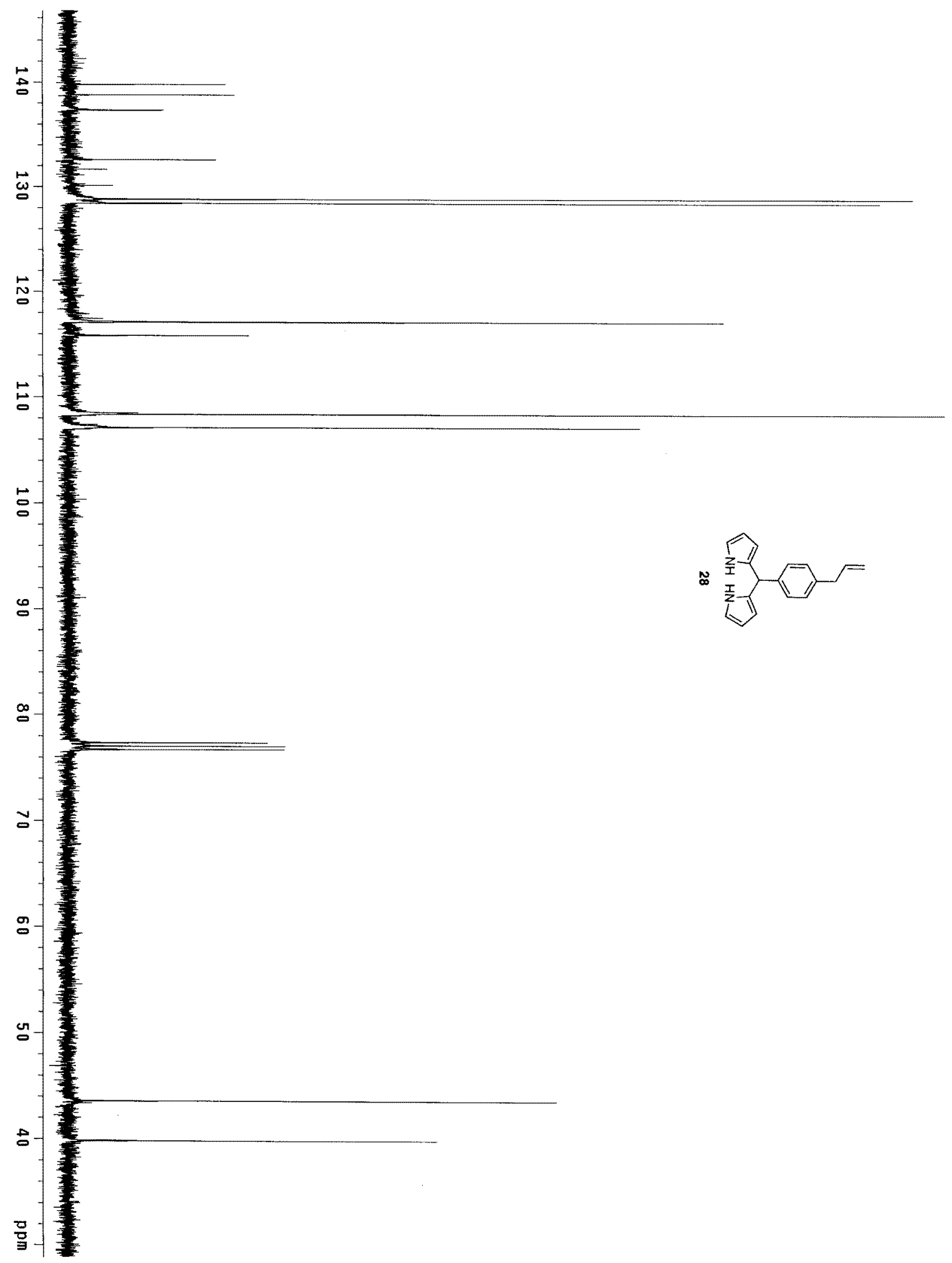




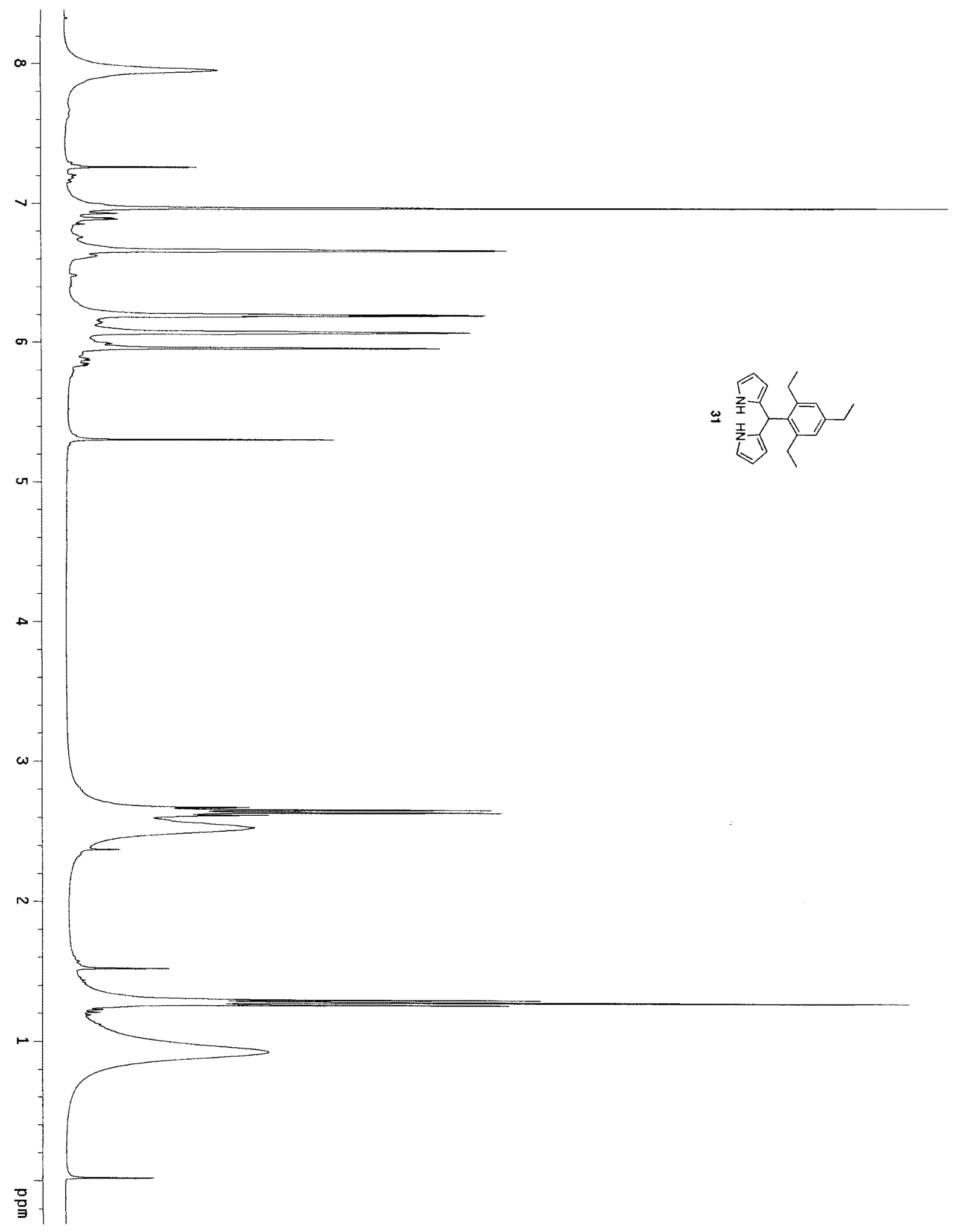




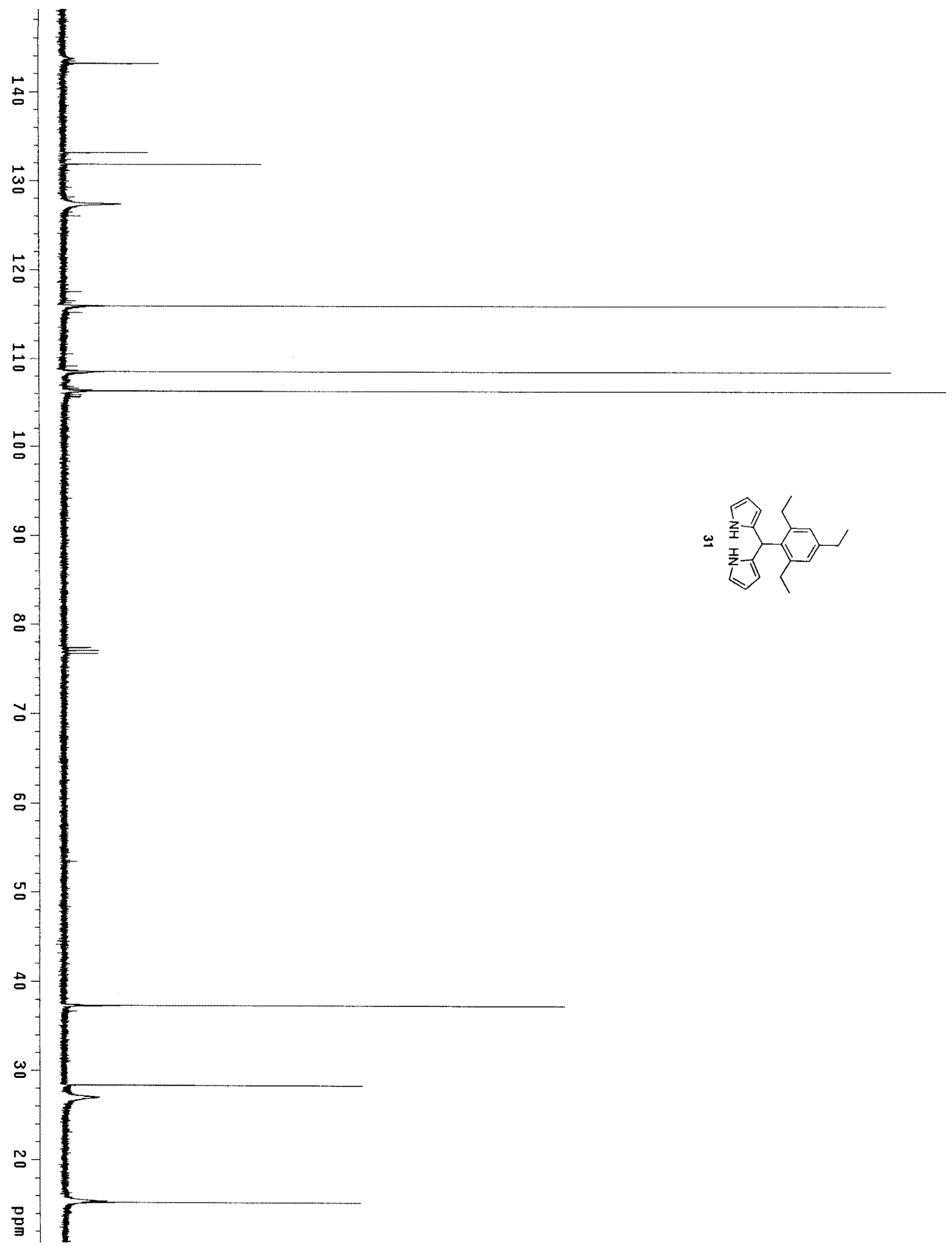




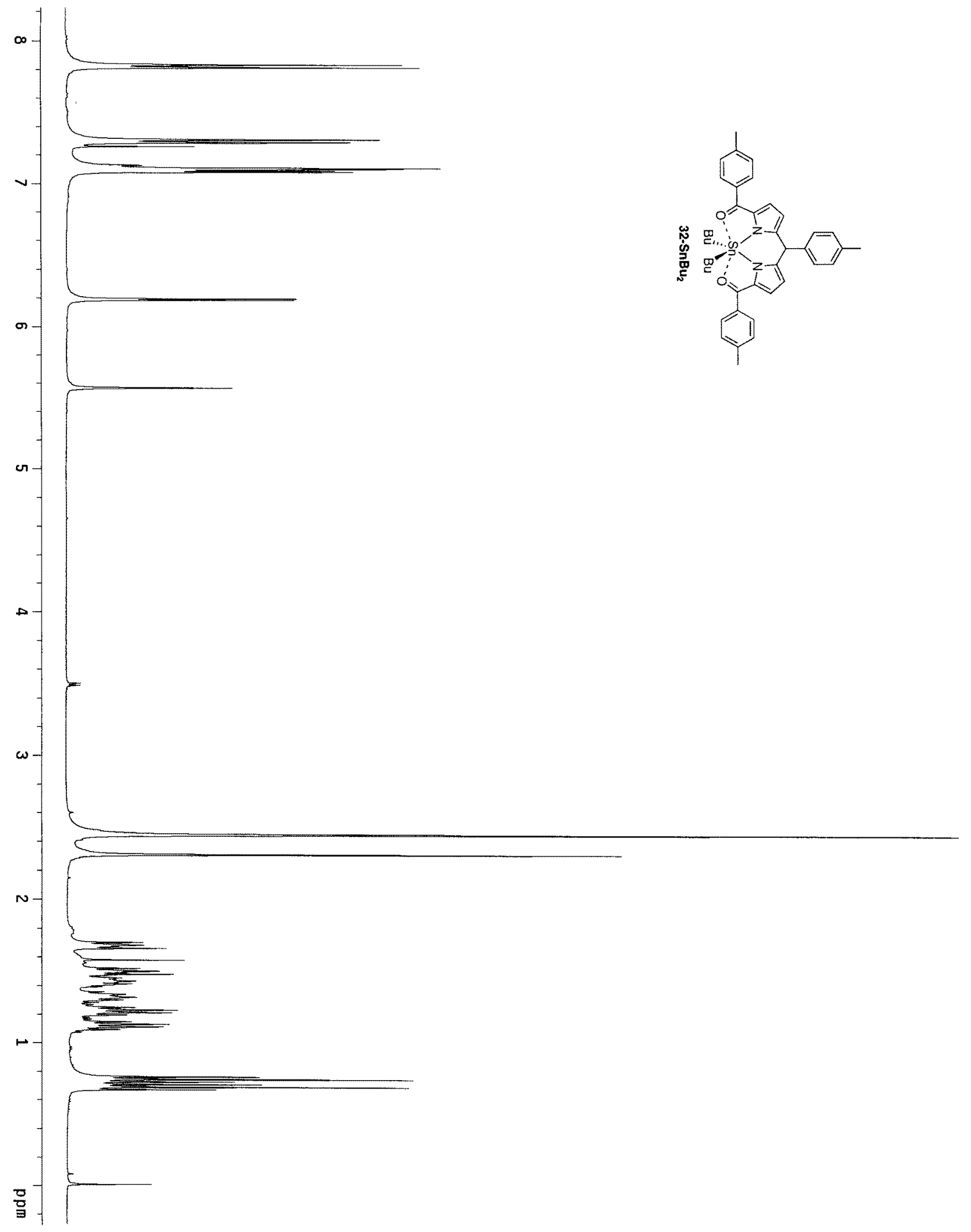




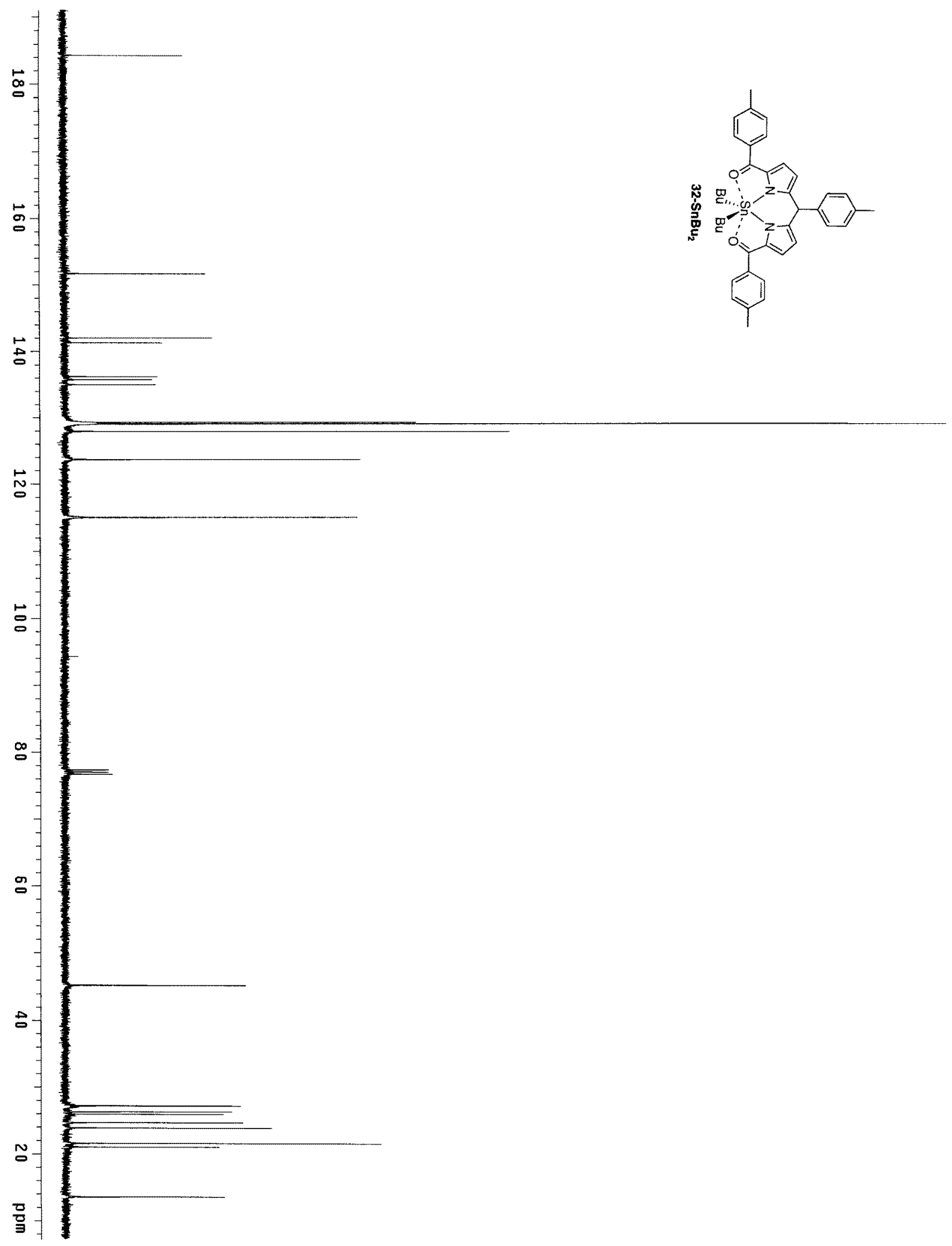




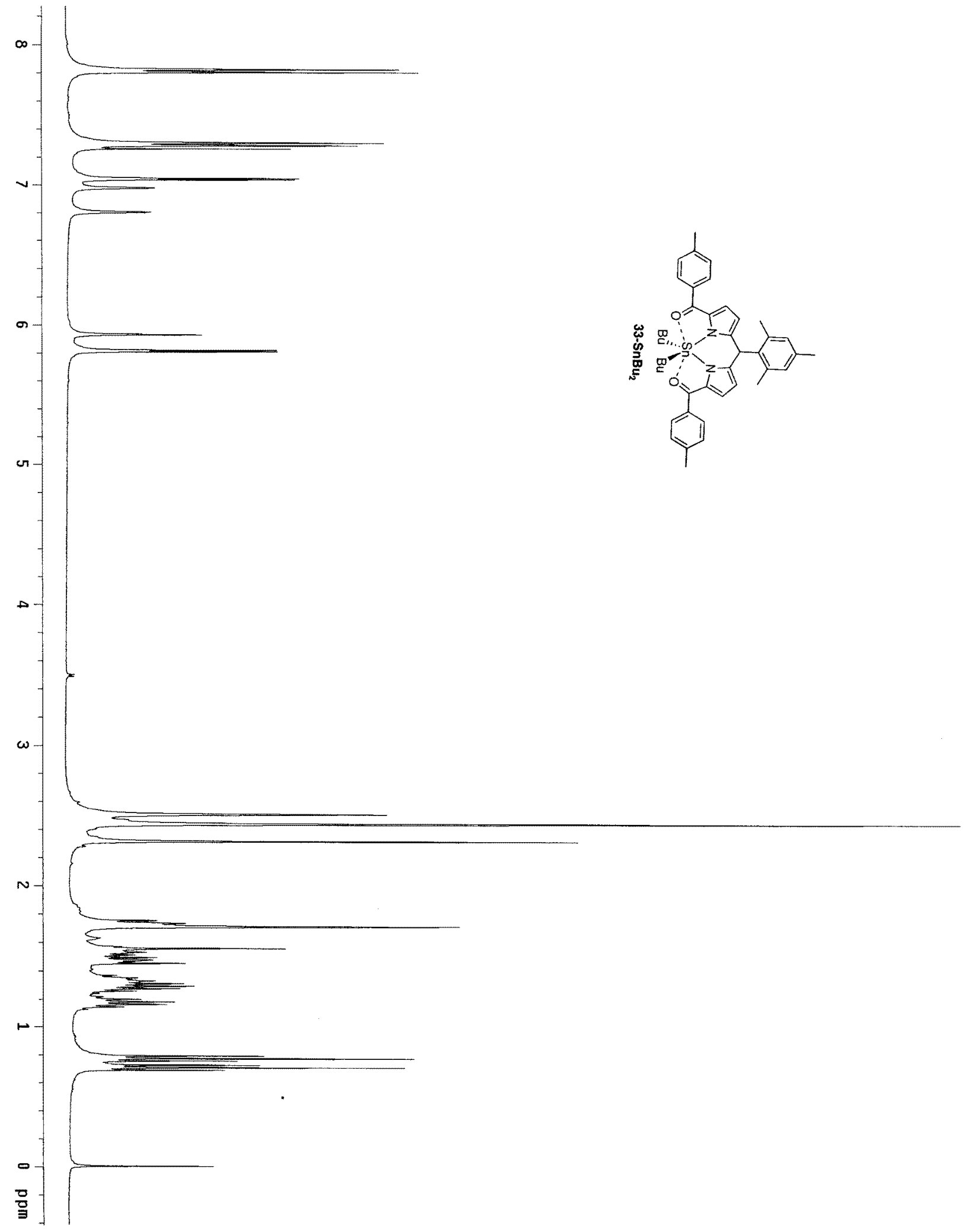




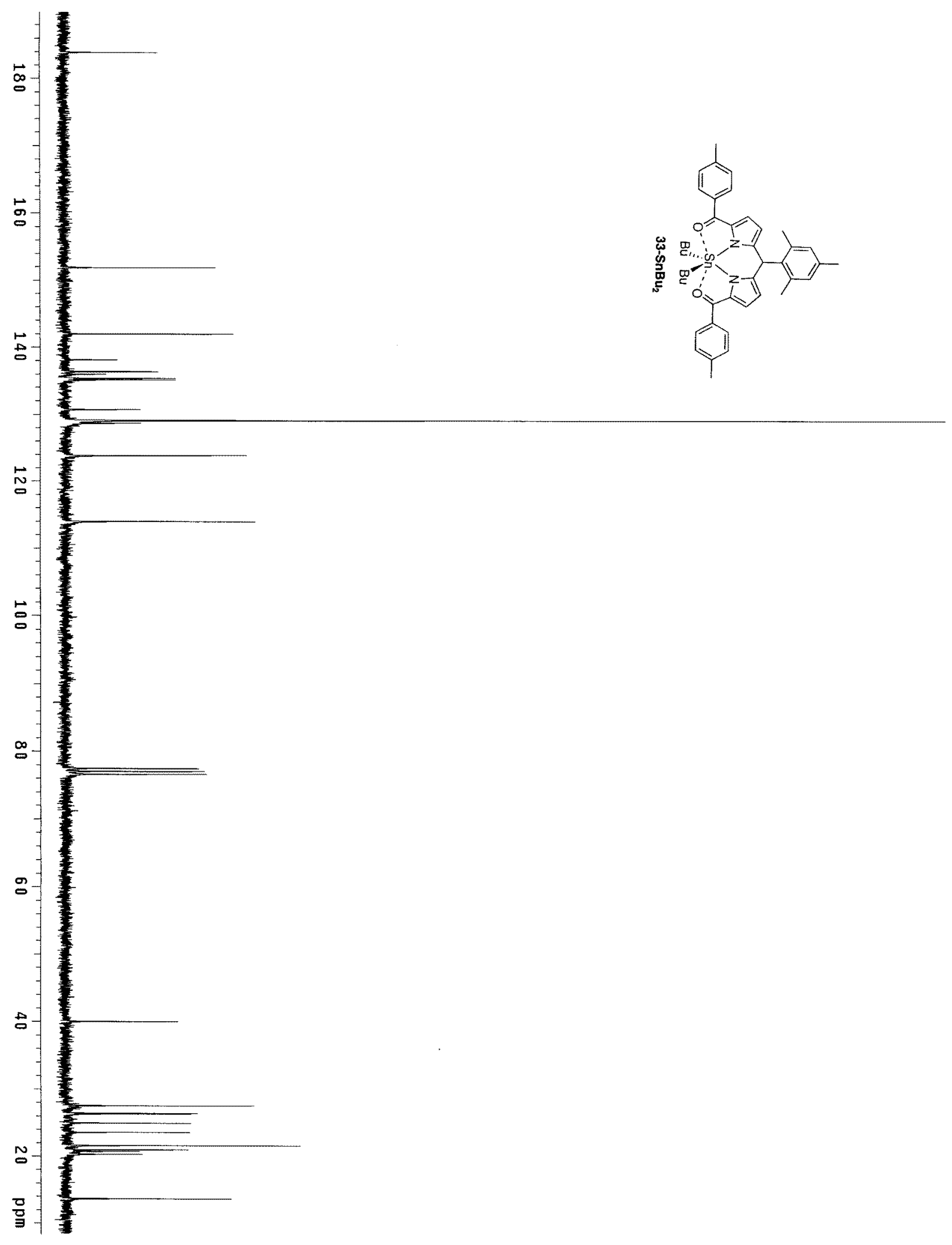




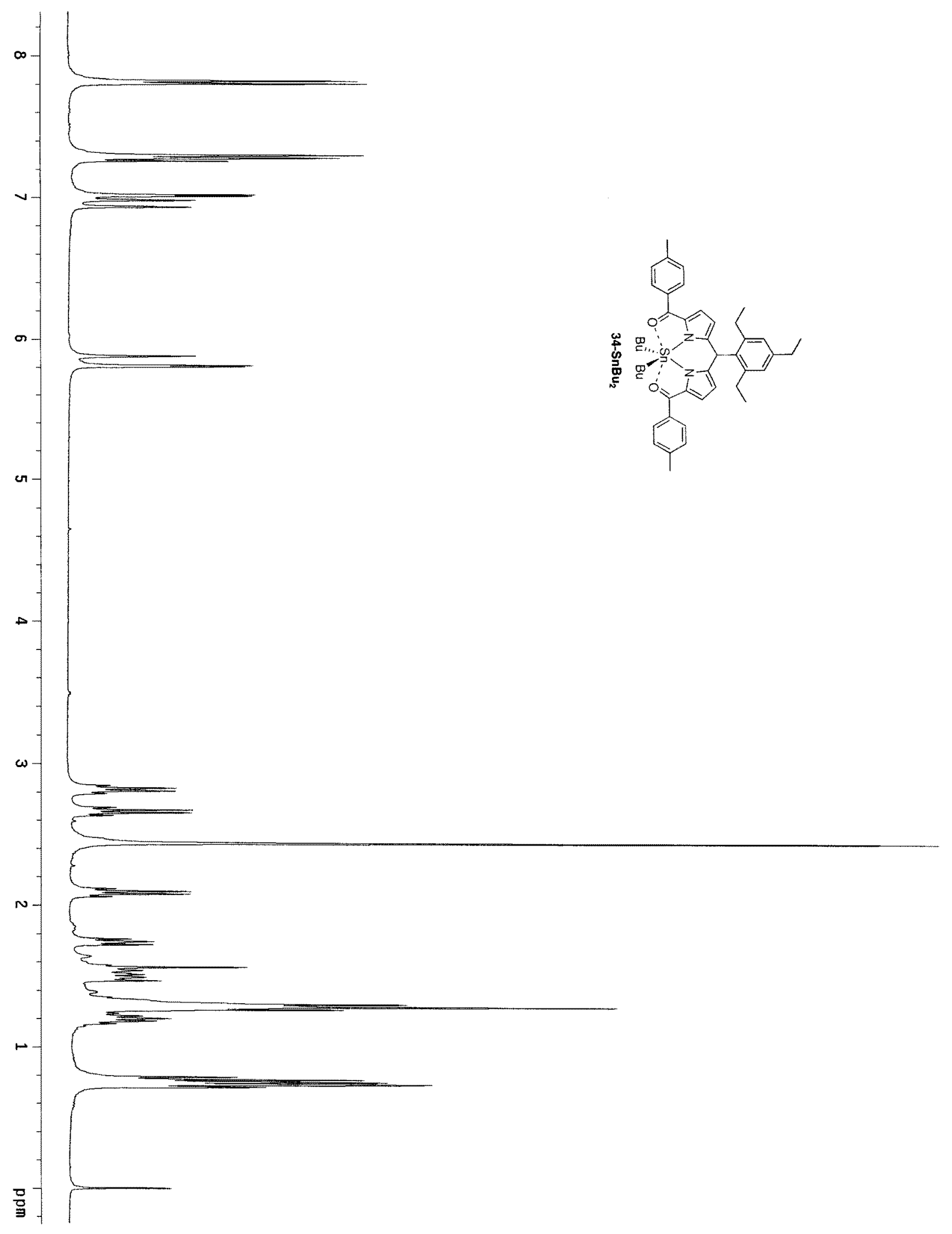




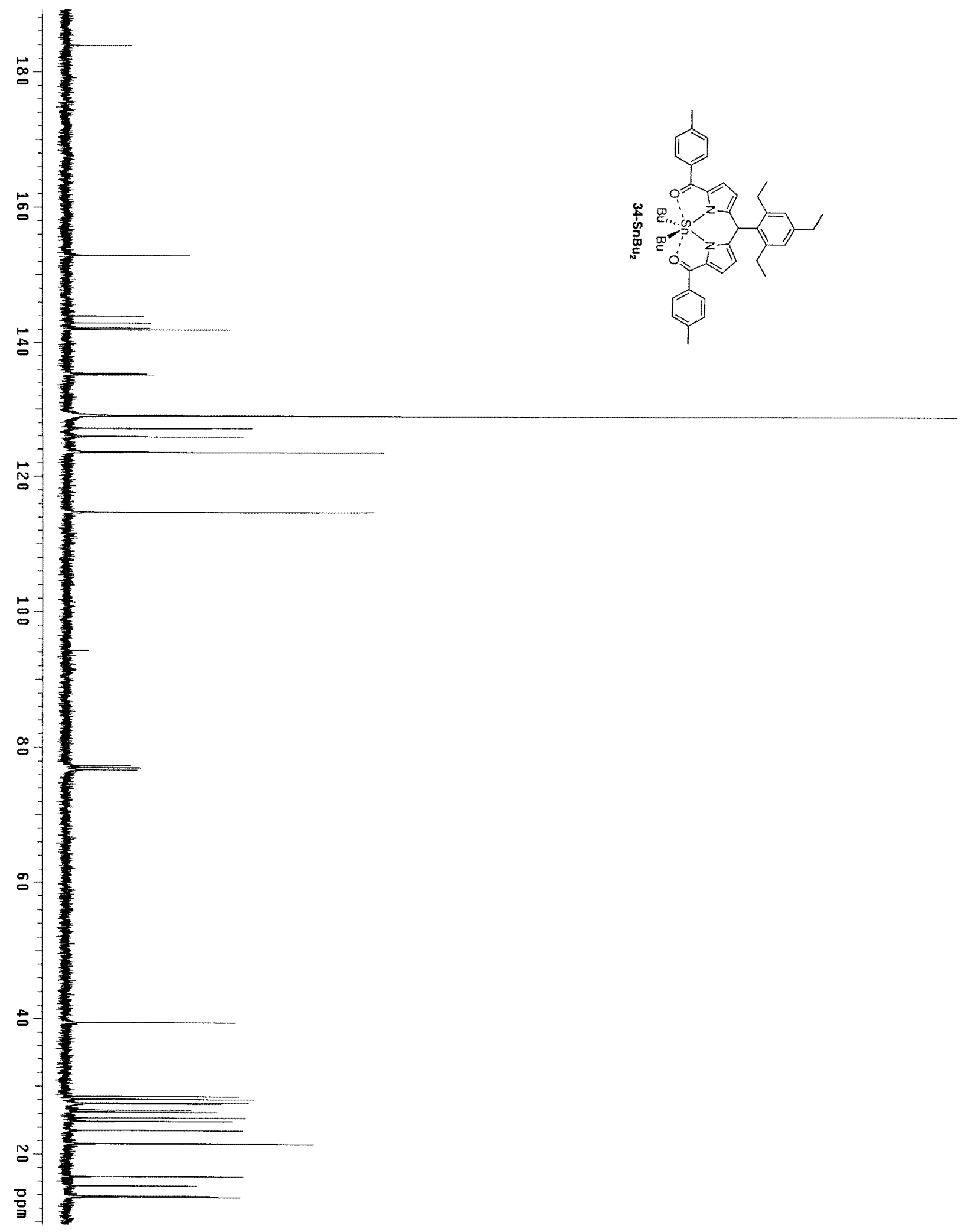




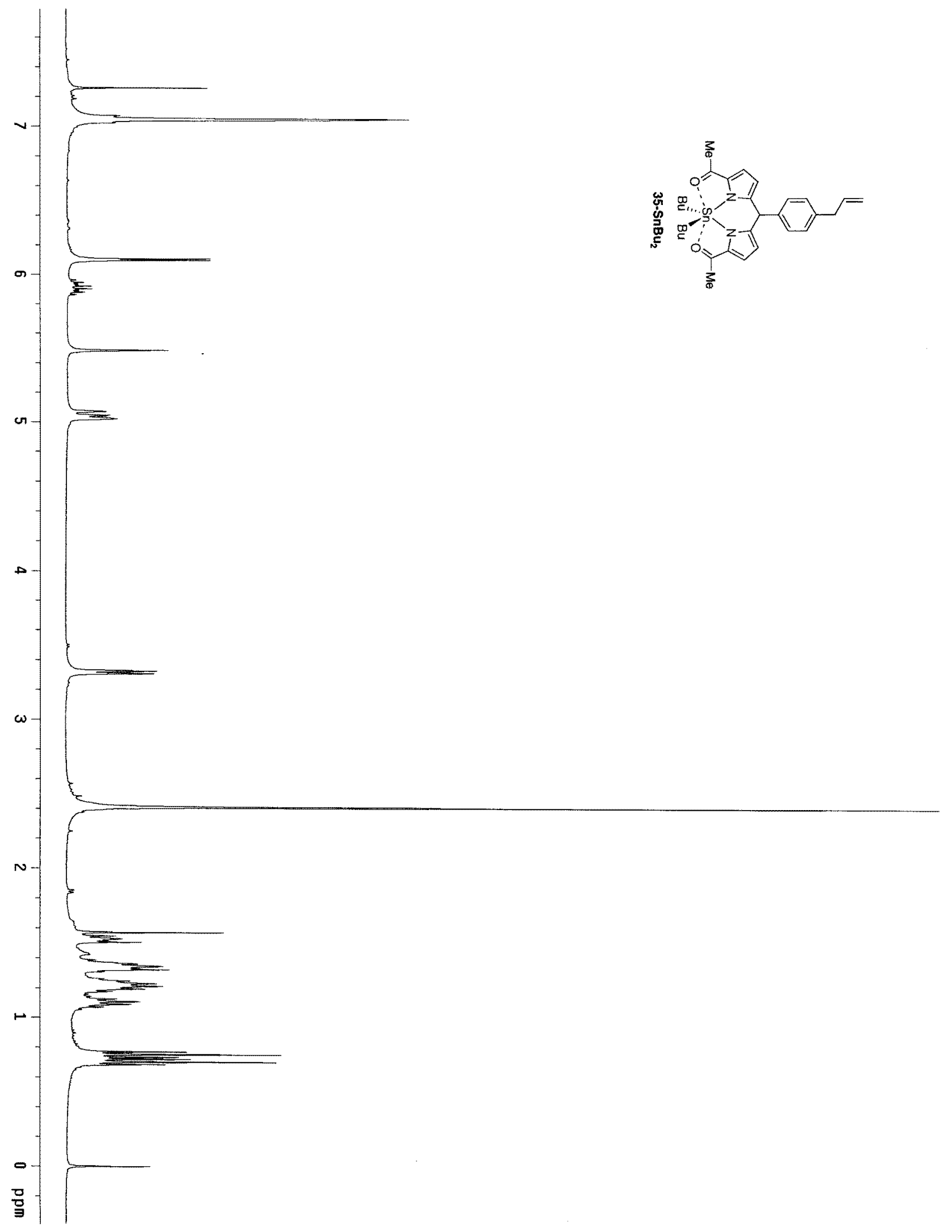




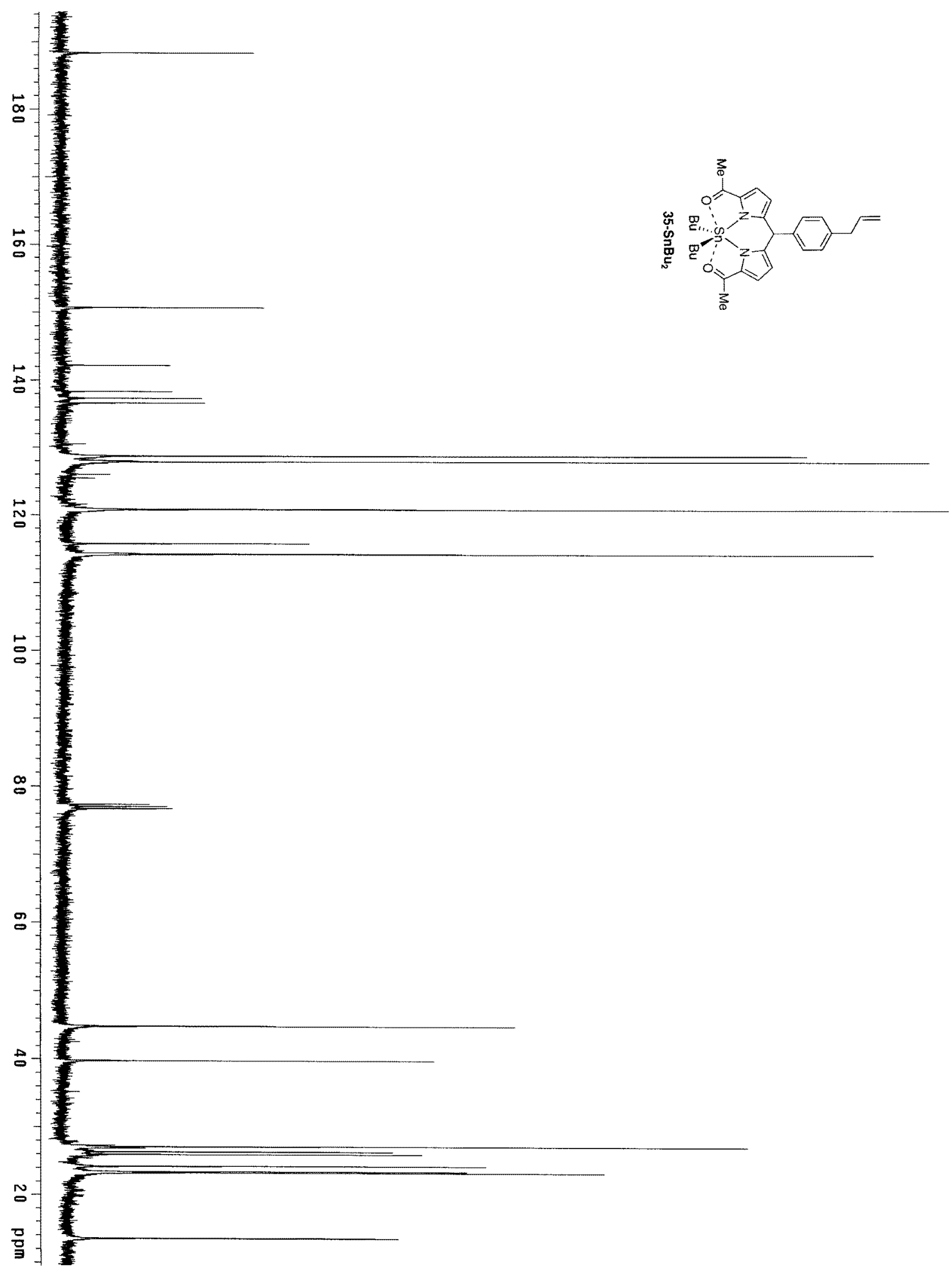

S-63 


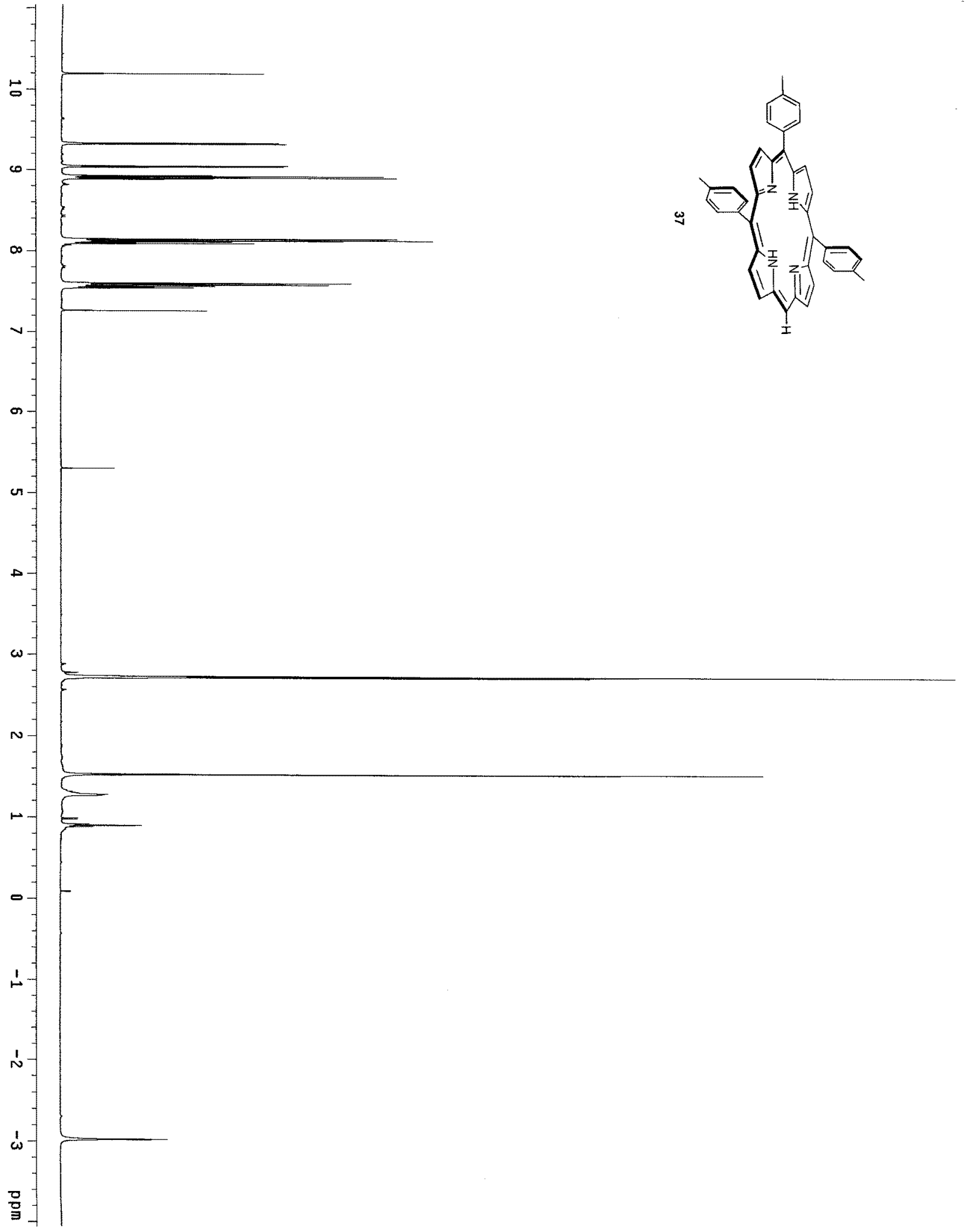




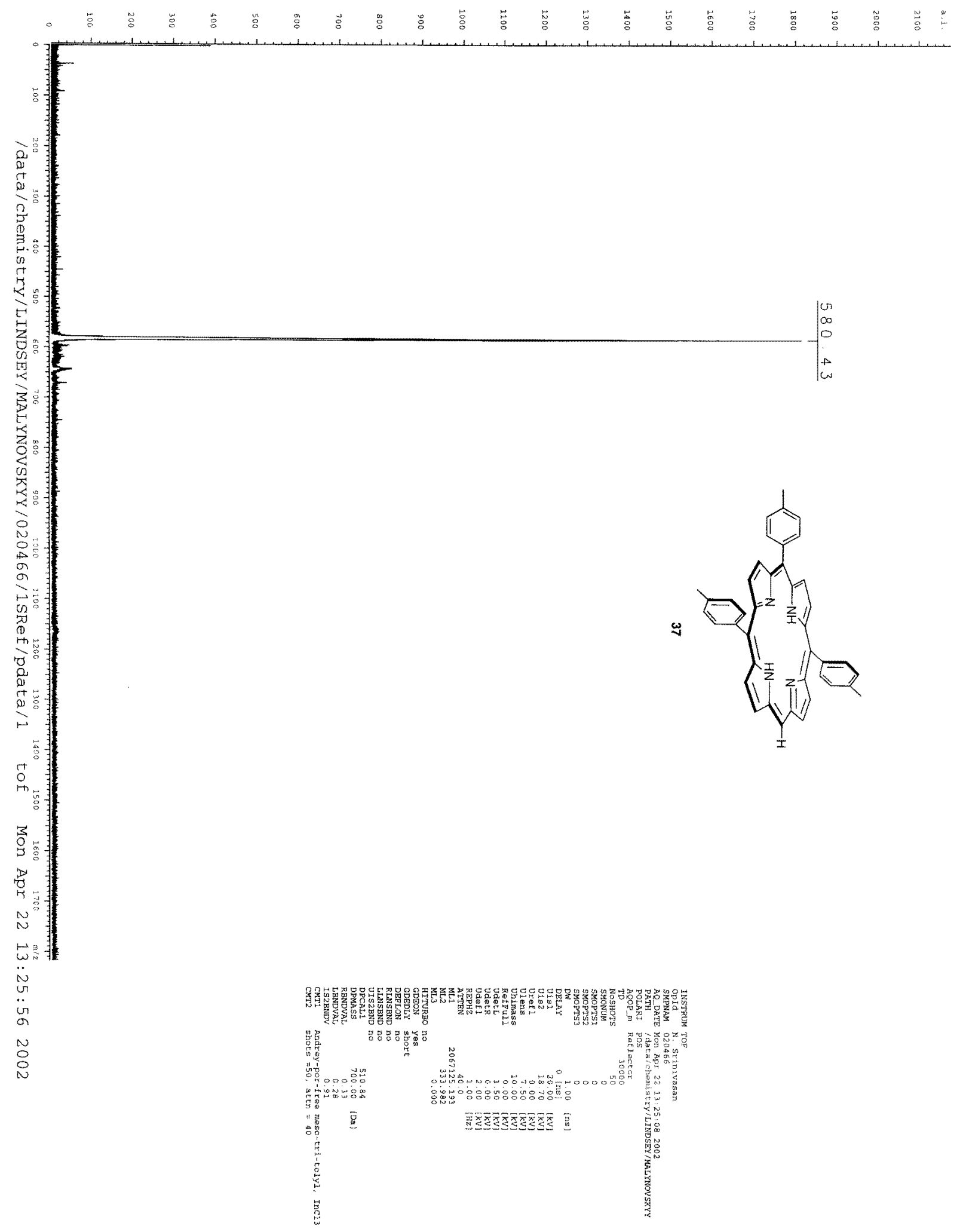




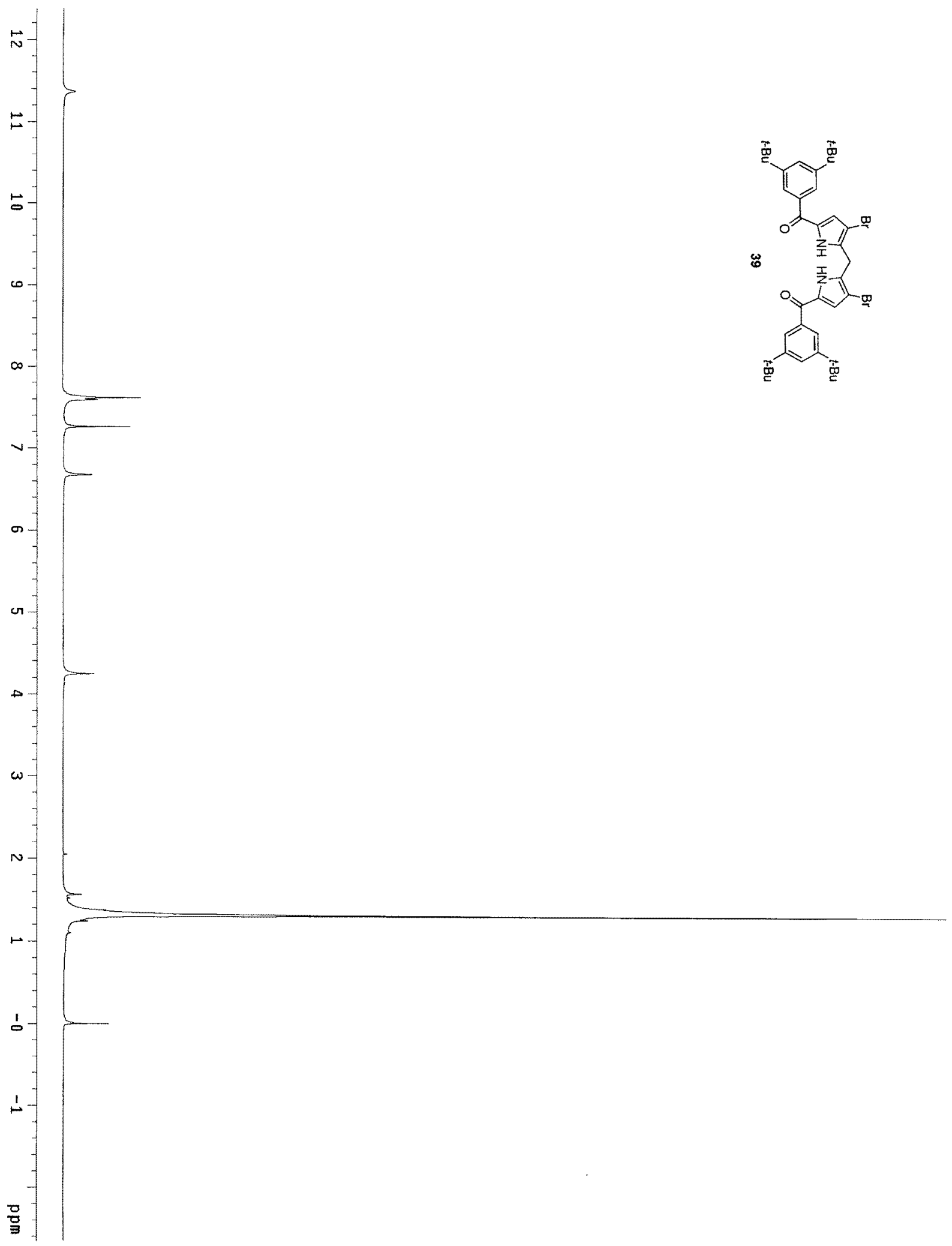




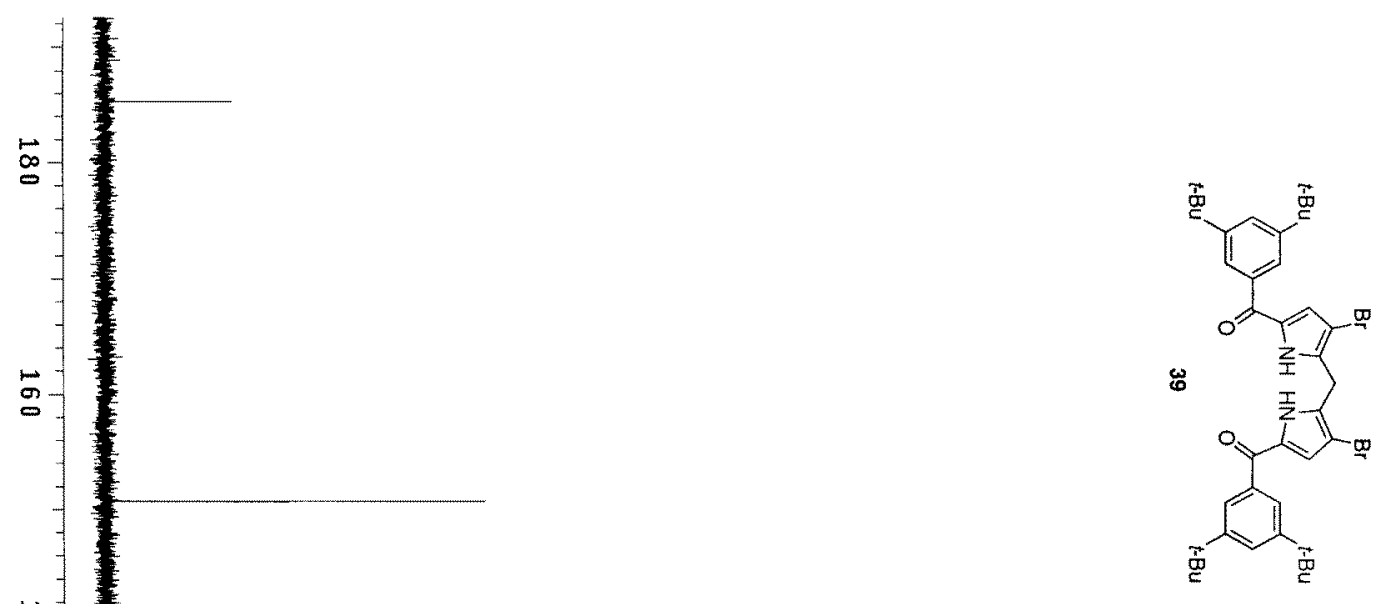

$\stackrel{+}{\mathrm{B}}$

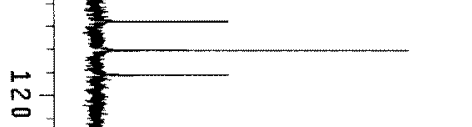

$\therefore$

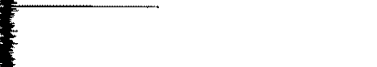

$\stackrel{\circ}{\circ}$

翌

a

-

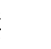




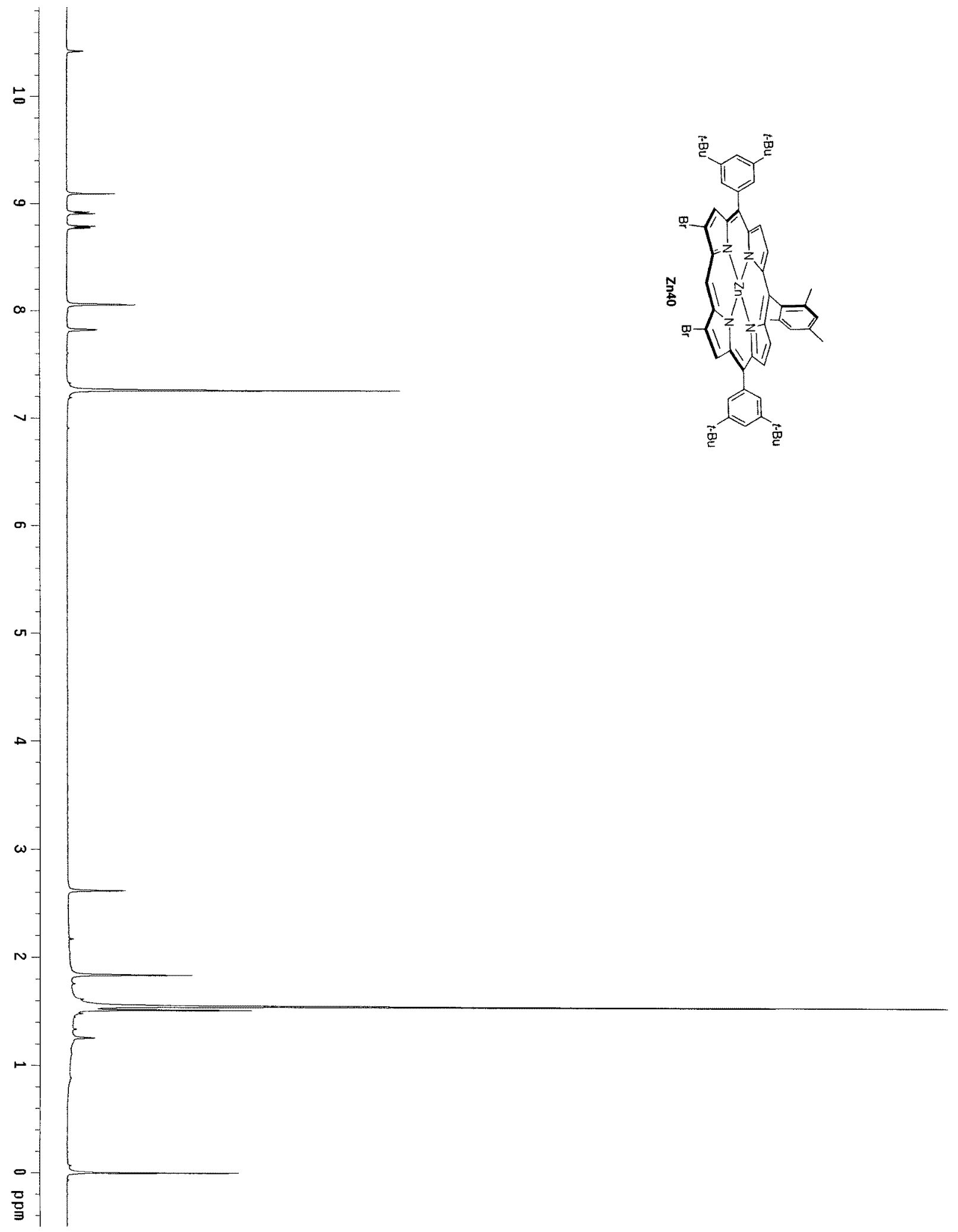




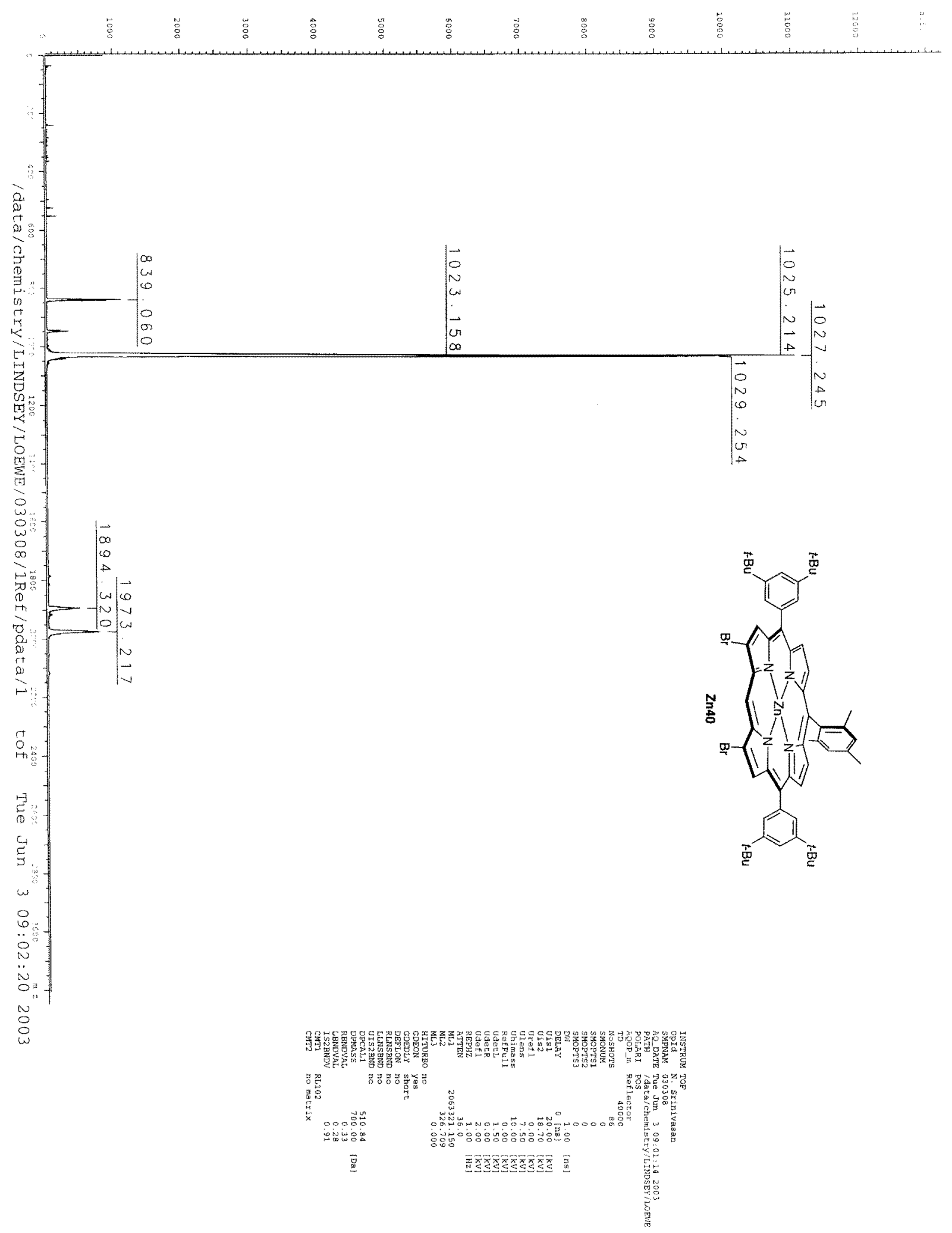




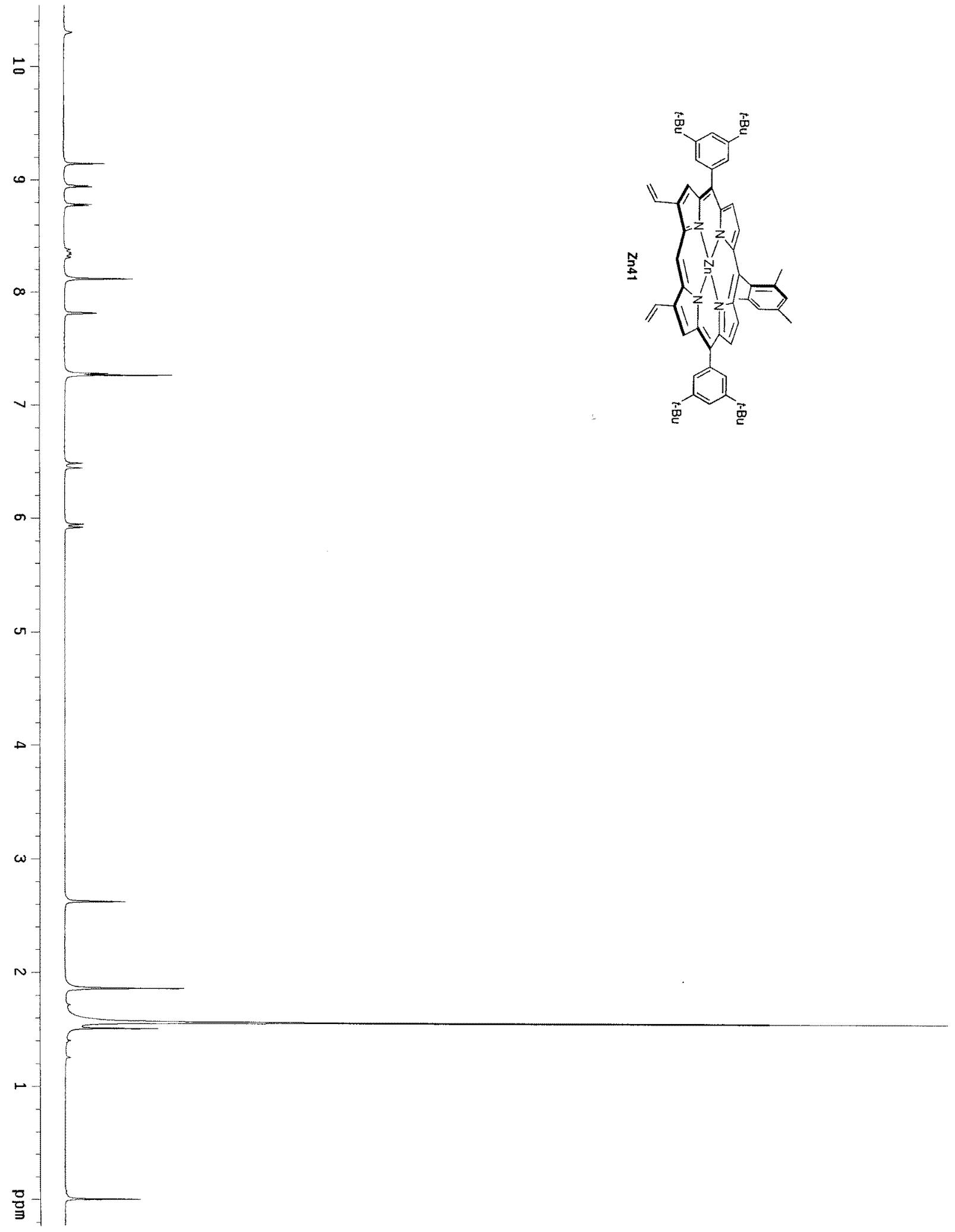




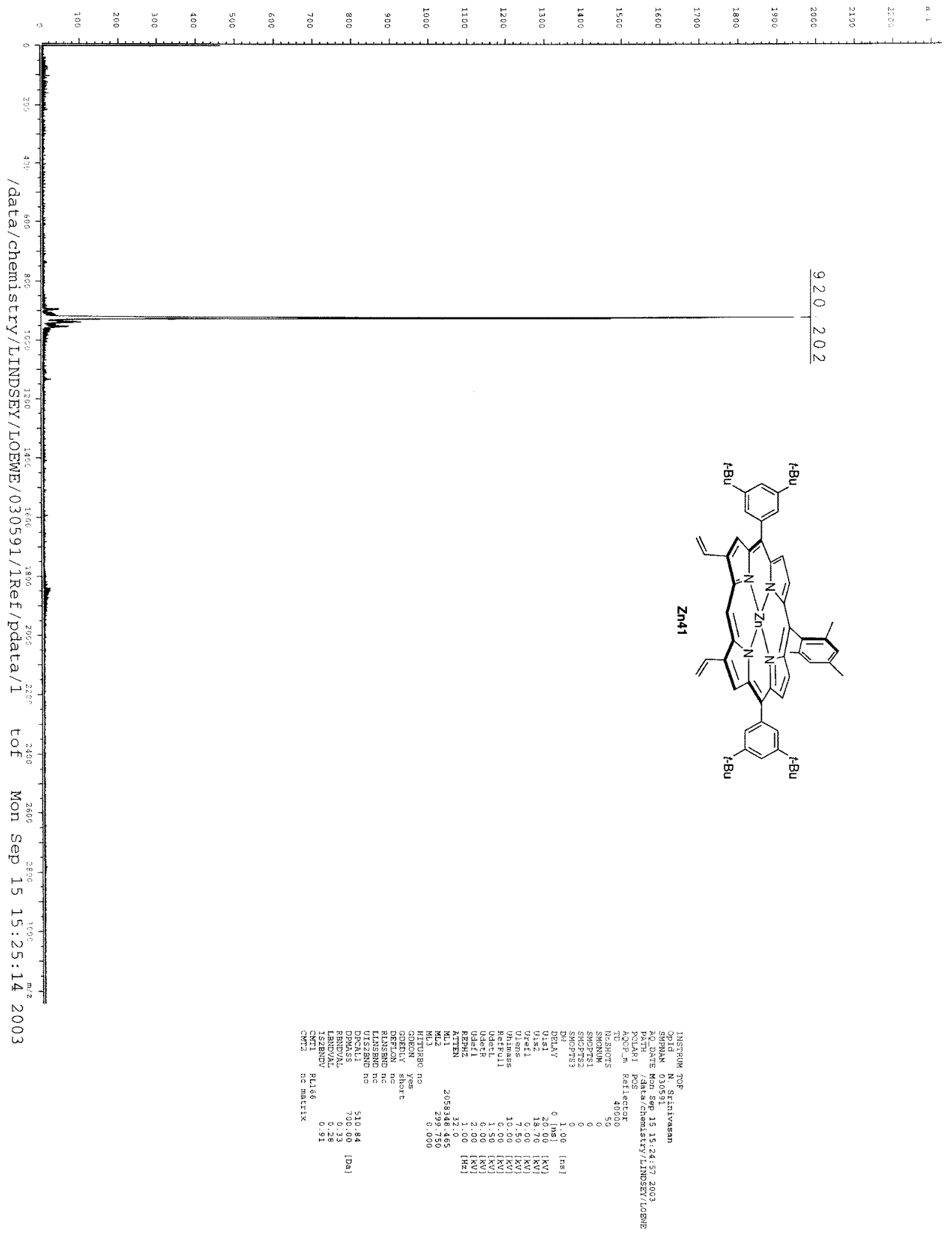




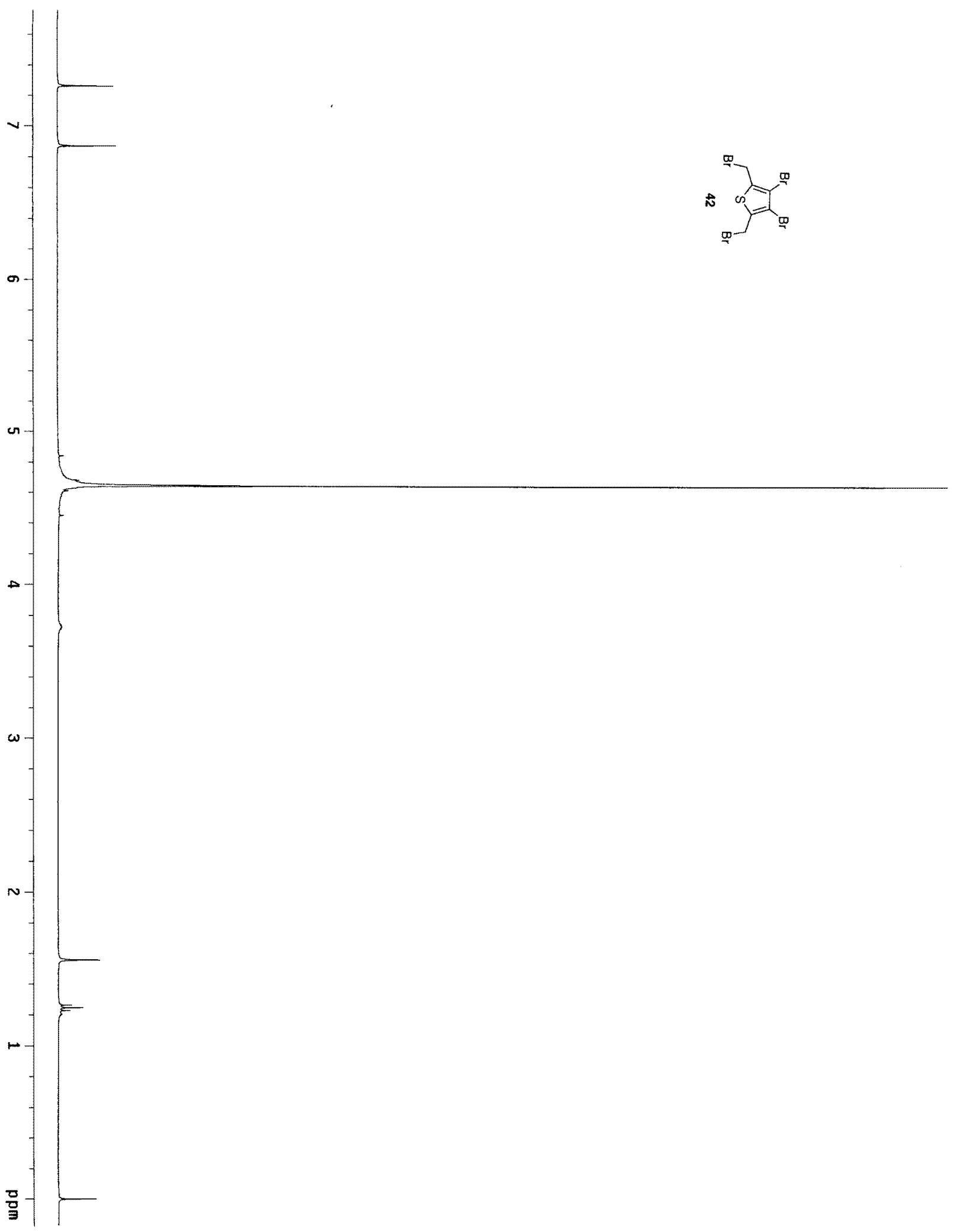




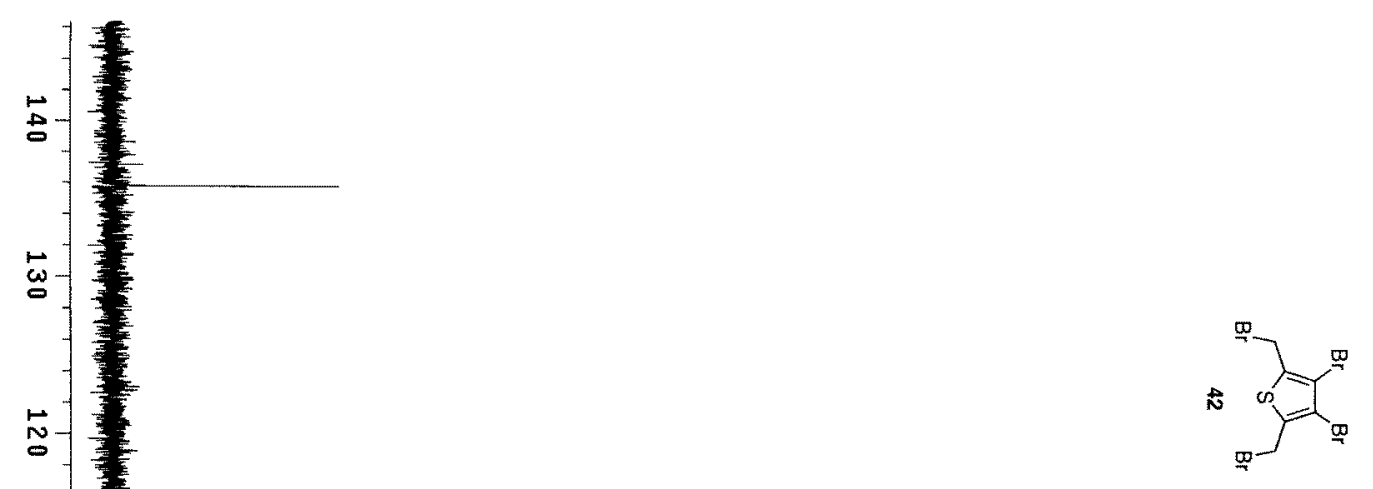

$\stackrel{5}{\circ}$

뭉

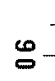

$\stackrel{\infty}{\circ}$

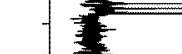

$\therefore-$

a

ㅇ.

a

a

$-1$

무의

S-73 


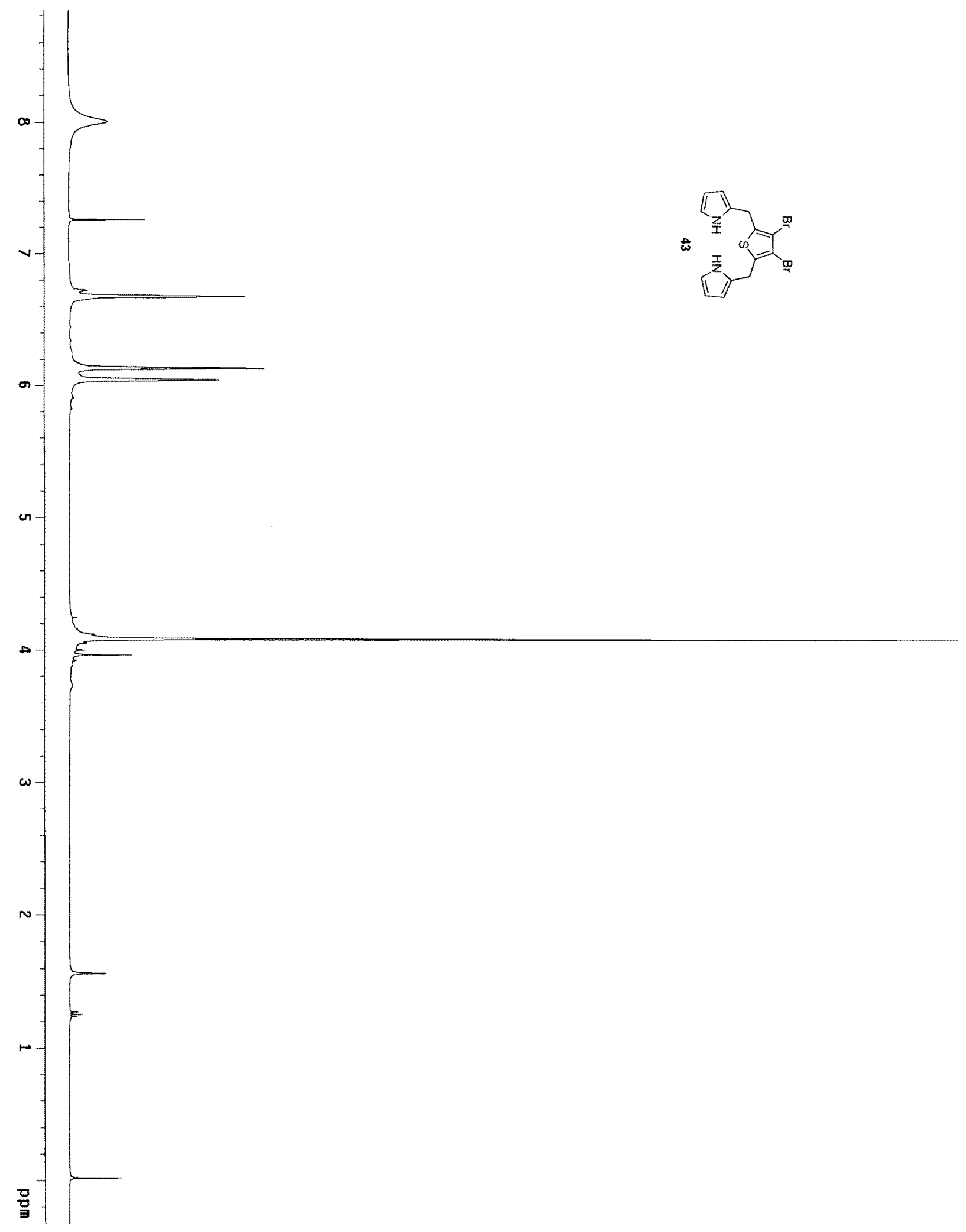




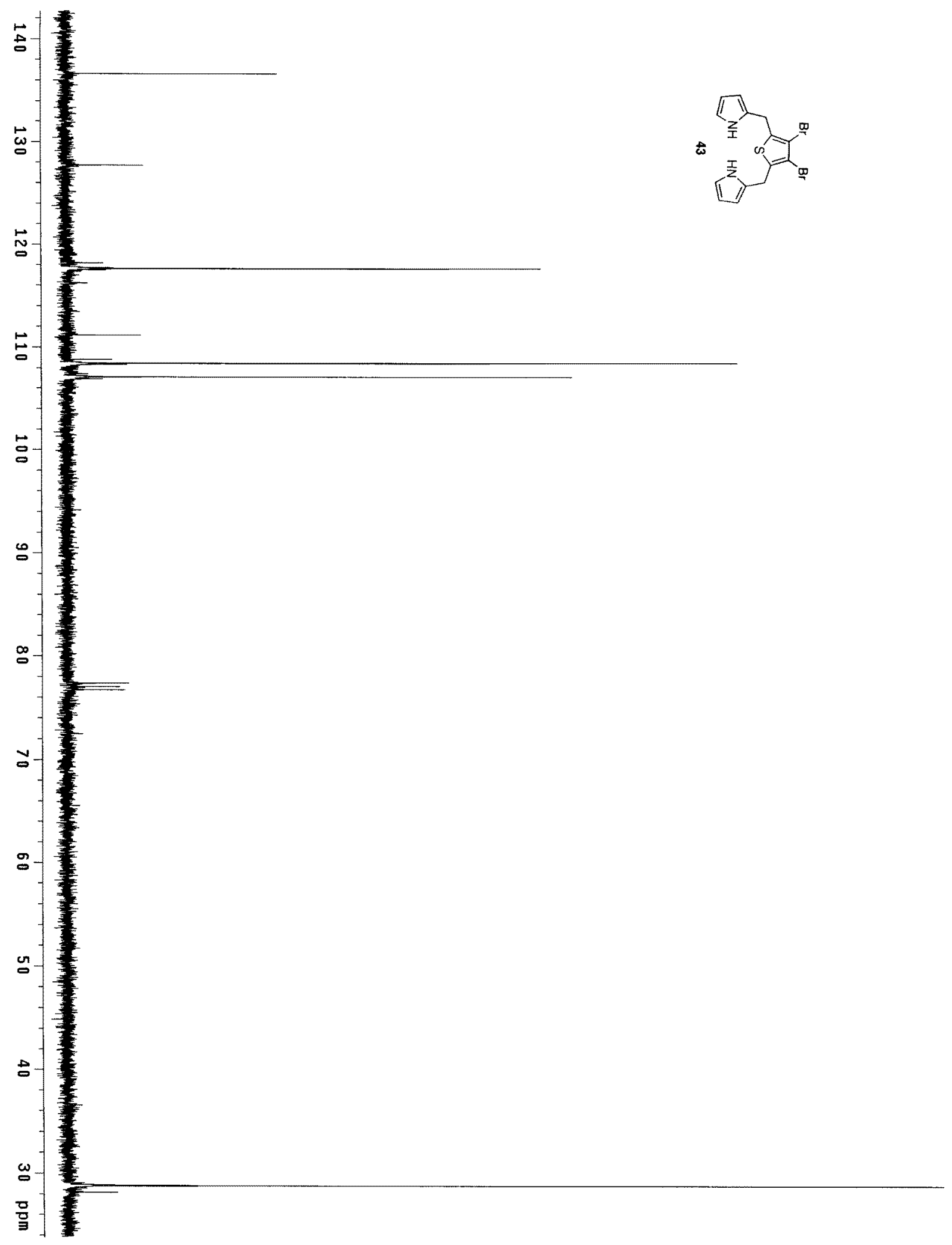

S-75 


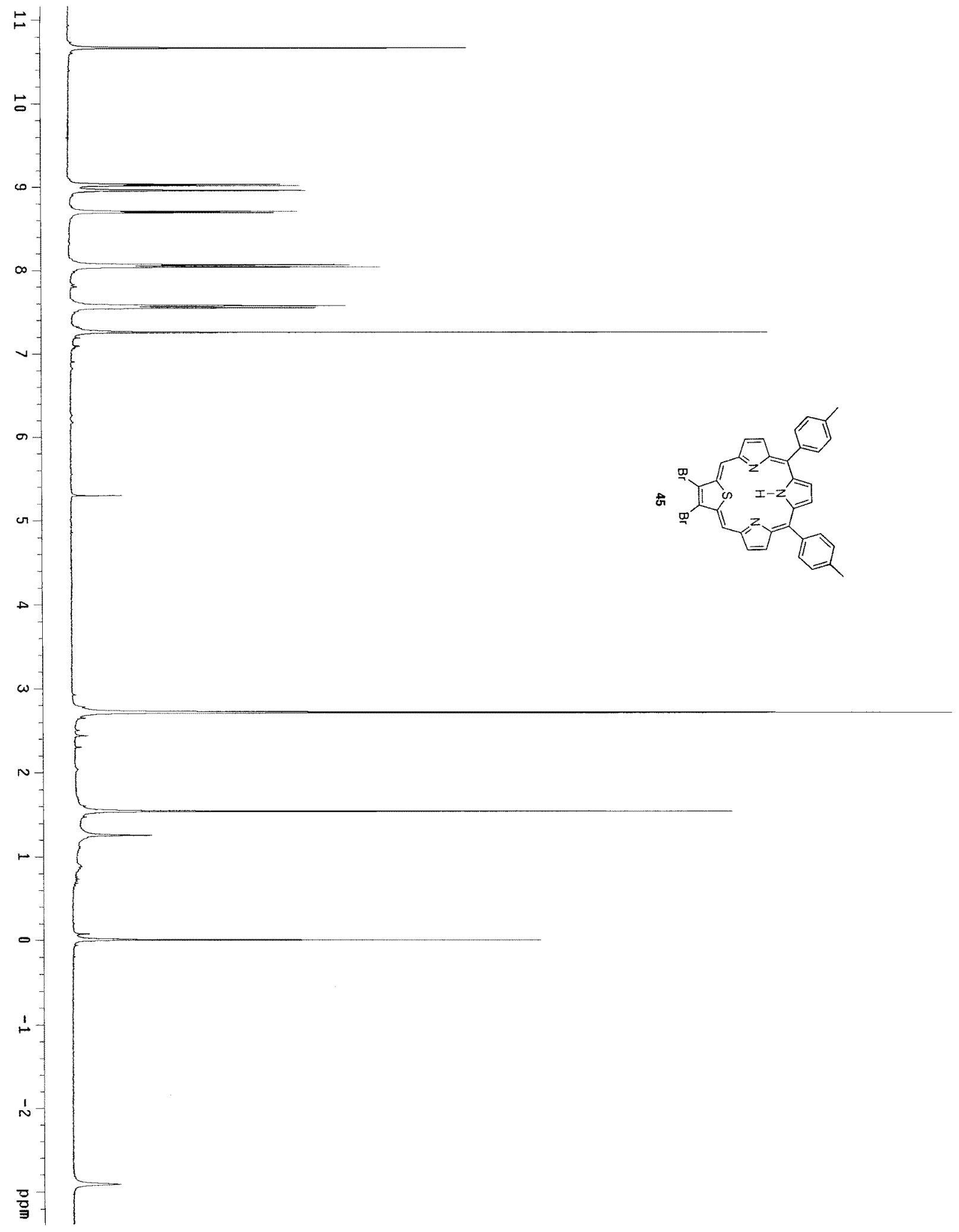




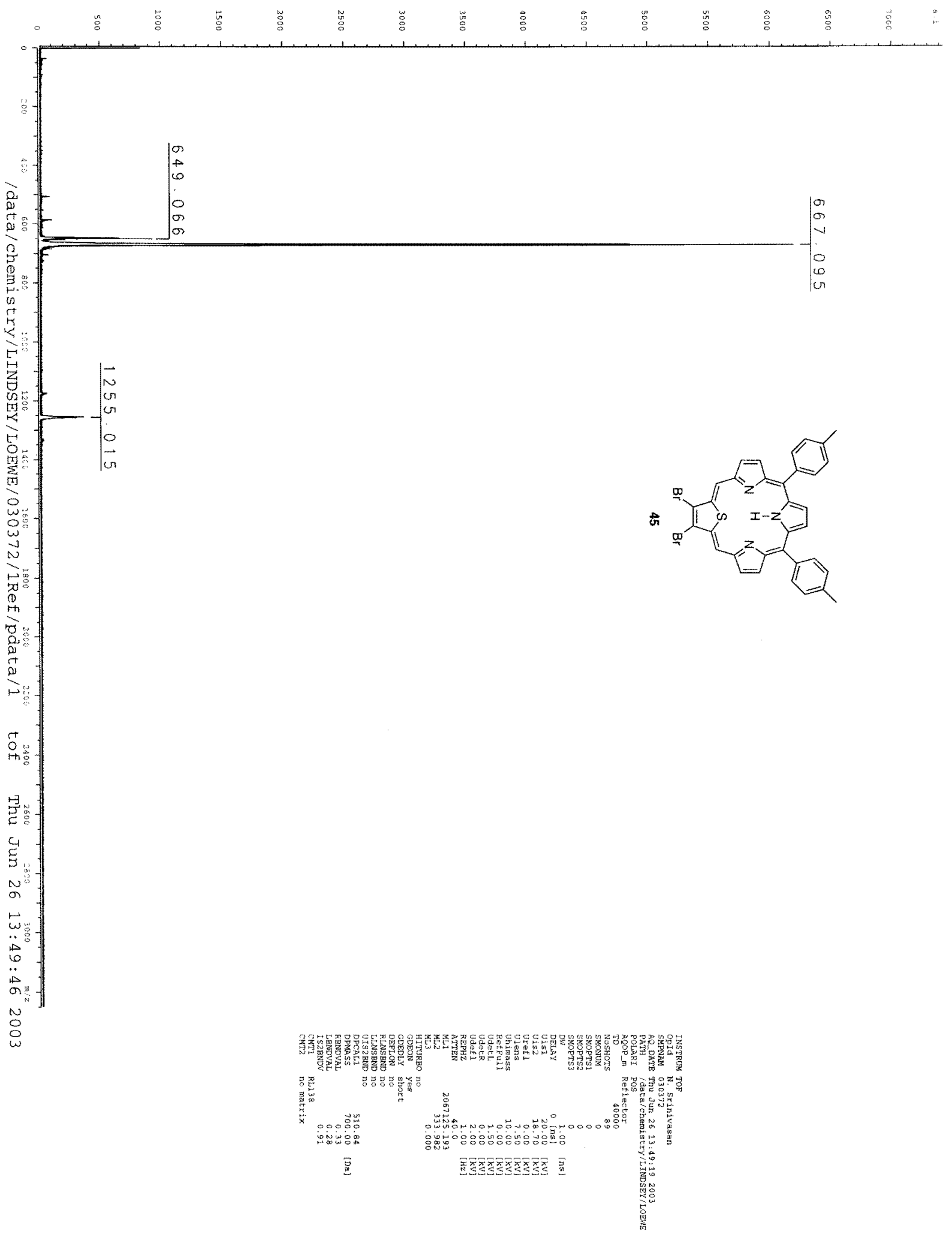

